$\mathrm{LTH} / 689$

UCB-PTH-06/02

LBNL-59632

\title{
Proton Decay, Yukawa Couplings and Underlying Gauge Symmetry in String Theory
}

\author{
${ }^{a}$ Radu Tatar and ${ }^{b}$ Taizan Watari \\ ${ }^{a}$ Division of Theoretical Physics, Department of Mathematical Sciences \\ The University of Liverpool, Liverpool,L69 3BX, England, U.K. \\ rtatar@liverpool.ac.uk \\ ${ }^{b}$ Department of Physics and Lawrence Berkeley National Laboratory, \\ University of California, Berkeley, CA 94720, USA \\ TWatari@lbl.gov
}

\begin{abstract}
In string theory, massless particles often originate from a symmetry breaking of a large gauge symmetry $G$ to its subgroup $H$. The absence of dimension- 4 proton decay in supersymmetric theories suggests that $(\bar{D}, L)$ are different from $\bar{H}(\overline{\mathbf{5}})$ in their origins. In this article, we consider a possibility that they come from different irreducible components in $\mathfrak{g} / \mathfrak{h}$. Requiring that all the Yukawa coupling constants of quarks and leptons be generated from the super Yang-Mills interactions of $G$, we found in the context of Georgi-Glashow $H=\mathrm{SU}(5)$ unification that the minimal choice of $G$ is $E_{7}$ and $E_{8}$ is the only alternative. This idea is systematically implemented in Heterotic String, $\mathrm{M}$ theory and $\mathrm{F}$ theory, confirming the absence of dimension 4 proton decay operators. Not only $H=\mathrm{SU}(5)$ but also $G$ constrain operators of effective field theories, providing non-trivial information.
\end{abstract}




\section{Introduction}

String theory is a well-formulated theory of quantum gravity, and it is also known to be able to (almost) realize the (supersymmetric) standard model of particle physics at lowenergies [1, 2, 3, 4, 15, 6, 7, 8]. Since 1990's, the understanding of string theory has improved dramatically, and an enormous variety of string vacua have been discovered. But, too much variety in string vacua and too much success in describing the standard model with string theory might also imply that there is no hope to obtain testable predictions from string theory in a near future.

Although the current string theory has a plethora of vacua, there is one thing in common among them. One of the most intriguing features of string theory is that it supports higherdimensional gauge theories with 16 supersymmetry charges where scalar, fermion and vector fields are unified. Thus, at least at high energies, the interactions of quarks, leptons and Higgs fields are under the constraint of the gauge principle. String theory can realize a large gauge group $G$ and this symmetry can be broken down spontaneously to a subgroup $H$ such as SU(5), either by vector bundles in Heterotic theory or by resolution of singularities in $\mathrm{M}$ / F theory. Particles in low-energy spectrum charged under $H$ arise from $\mathfrak{g} / \mathfrak{h}$ part of the high-energy supersymmetric vector multiplet. The old ideas of Higgs multiplets [9] or quarks and leptons [10] coming out of a coset space $\mathfrak{g} / \mathfrak{h}$ or its complexification are realized naturally in string theory.

In the present work, we focus on the underlying large gauge symmetry $G$ and 16 supercharges. The usage of the non-linearly realized symmetry $G$ as a constraint of low-energy interactions is an idea that dates back to 1960 's. When the symmetry $G$ is a gauge symmetry, and it is combined with the underlying 16-charge supersymmetry, all the interactions of quarks and leptons are constrained by the non-linearly realized gauge symmetry. We are not obtaining numerical predictions, as there is a lot of room for corrections and arbitrariness to come in. Our approach is to use the underlying symmetries as a powerful tool at the level of knowing whether certain interactions exist or not.

We start from an assumption that the Yukawa couplings originate from supersymmetric Yang-Mills interactions of a large symmetry $G$ that contains $H=\mathrm{SU}(5)$. Theories with lowenergy supersymmetry should not have dimension-4 proton decay operators, which means that there must be some difference in the origins of $\overline{\mathbf{5}}=(\bar{D}, L)$ and $\bar{H}(\overline{\mathbf{5}})$ multiplets, although their representations under the unified $\mathrm{SU}(5)_{\text {GUT }}$ gauge group are the same. Thus, we introduce another assumption that they have different origins in $\mathfrak{g} / \mathfrak{h}$, so that these two 
multiplets have different interactions. Then we prove in section 2 that $G$ has to be at least $E_{7}$. The argument that leads to this statement does not depend on any implementation in string theory and it is only based on commutation relations and irreducible decomposition of Lie algebra. String theory supports $E_{7}$ symmetry in Heterotic, M- and F-theory. $G=E_{8}$ contains the minimal $E_{7}$ gauge symmetry and is also supported by string theory. They are the only possibilities for the underlying gauge symmetry in String Theory. The only way to get these groups other than in Heterotic theory is to go to the nonperturbative limits of Type IIA or Type IIB, which are $\mathrm{M}$ theory and $\mathrm{F}$ theory, respectively. If one works in the perturbative type IIA or type IIB, the vacua with intersecting D-branes cannot be used for realising these symmetries. We show that there are overall seven possibilities in the way low-energy particles are identified in the coset space $\mathfrak{e}_{7} / \mathfrak{s u}(5)$ or $\mathfrak{e}_{8} / \mathfrak{s u}(5)$.

The idea presented in section 2 is realized explicitly in Calabi-Yau compactification of Heterotic theory in section 3 , in $G_{2}$-holonomy compactification of eleven-dimensional supergravity in section 4 and finally in elliptic Calabi-Yau compactification of F-theory in section 5. For instance, in Heterotic compactification, we see that the absence of dimension-4 proton decay is guaranteed (at the perturbative level) when a stable rank-5 vector bundle $V_{5}$ has a negative-slope sub-bundle with certain properties. Origins of low-energy multiplets are identified for each symmetry-breaking pattern, in each section. Discussion in section 2 guarantees that Yukawa couplings of charged leptons, down-type and up-type quarks are generated from the super Yang-Mills interactions, and in some cases, Dirac neutrino Yukawa couplings as well. Many yet-to-be answered questions are raised in this article, and at the same time, some predictions are also derived, using the constraint of the underlying gauge symmetry and supersymmetry.

Reading guide: The only prerequisite for section 2 is some knowledge in Lie algebra, and this section will be readable without details of string theory and it presents the key idea of this article. The later three sections for Heterotic (section 3) M- (section 4) and F-theory (section 5) are almost mutually independent, but assume the contents of section 2 For those who do not want to read all through the three sections, summary of physics is given in section 6.

\section{Bottom-up Classification of the Origins of Matters}

In this article, we only consider Georgi-Glashow SU(5) Gut unification. A good bottom-tau Yukawa unification and a similar degree of hierarchy among Yukawa couplings of down-type 
quarks and charged leptons are consistent with this unification. The disparity between the large mixing angles in the neutrino oscillations and the small mixing angles in the quark sector can be attributed to the difference in the nature of $\mathrm{SU}(5)_{\mathrm{GUT}^{-}} \mathbf{1 0}=(Q, \bar{U}, \bar{E})$ representations containing quark doublets $Q$ and $\mathrm{SU}(5)_{\mathrm{GUT}^{-}} \overline{\mathbf{5}}=(\bar{D}, L)$ representations containing lepton doublets $L$ [12, 13]. All these are the motivations for us to consider $\mathrm{SU}(5)_{\text {GUT }}$ unification. Since no extra gauge symmetry prevents right-handed neutrinos from having large Majorana masses, tiny neutrino masses may be explained by the see-saw mechanism [14, and this may be another good feature of the $\mathrm{SU}(5)_{\text {GUT }}$ unification. If the gauge group is larger, it has to be broken in a field-theoretical way. But, the analysis in this article can also be taken just as an example, and one can freely extend the analysis to other unification groups. Indeed, the above argument is not as rigorous as it might appear.

\subsection{Assumptions}

In order for us to be able to understand the Yukawa couplings better, we assume that all the Yukawa couplings of up-type and down-type quarks and charged leptons are generated from super Yang-Mills interactions of an underlying symmetry $G$, and all the low-energy multiplets are given their origins in the coset space $\mathfrak{g} / \mathfrak{s u}(5)$.

Another assumption that we introduce in this article is that $\overline{\mathbf{5}}=(\bar{D}, L)$ and $\bar{H}(\overline{\mathbf{5}})$ have distinct origins in the coset space $\mathfrak{g} / \mathfrak{s u}(5)$. This is to come up with models without dimension4 proton decay operators. Indeed, we always need Yukawa couplings of down-type quarks and charged leptons

$$
W \ni y \overline{\mathbf{5}} \cdot \mathbf{1 0} \cdot \bar{H}(\overline{\mathbf{5}})
$$

while the dimension- 4 proton decay operators should be absent ${ }^{1}$

$$
W \ni \overline{\mathbf{5}} \cdot \mathbf{1 0} \cdot \overline{\mathbf{5}}=\bar{D} \cdot \bar{U} \cdot \bar{D}+\bar{D} \cdot Q \cdot L+L \cdot \bar{E} \cdot L
$$

or protons would not remain stable. The operators (11) and (2) differ only by $\bar{H}(\overline{\mathbf{5}})$ in (11) and $\overline{5}$ in (2). If they are identified with different parts of the coset space $\mathfrak{g} / \mathfrak{h}$, then the operators (11) and (2) transform differently under some symmetry in $G$. Thus, such symmetry can kill the dangerous operators (2) while allowing the necessary Yukawa couplings (11). ${ }^{2}$ For

\footnotetext{
${ }^{1}$ If the supersymmetry breaking masses of the squark-slepton sector are much higher than the electroweak scale, the constraints on those operators are weaker. But, even for the supersymmetry breaking masses of order $10^{10} \mathrm{GeV}$, the coefficients of the operators (2) should be much smaller than unity.

${ }^{2}$ This assumption is to try to solve the dimension-4 proton decay problem by restricting the underlying symmetry $G$ and its breaking pattern. The other possibility that is not covered here is that those two multi-
} 
the moment being, we keep an open mind to all kinds of possibility for the choice of such symmetry. We shall see in this section that there are strong restrictions on the symmetry.

\subsection{Up-type Quark Yukawa Couplings from $E_{6}$ Symmetry}

In order to figure out the minimal underlying symmetry $G$, we proceed by enhancing $G$ a little by little on necessity basis. The enhanced symmetry provides both massless matters and their interactions. The 2 phenomenology assumptions constrain the origin of low-energy particles and the underlying symmetry $G$. The choice of $G$ and its breaking pattern can be determined purely in representation theory of Lie algebra, without paying much attention to the explicit implementation in string theories. The net chirality in the low-energy spectrum depends on topological aspects of geometry of compactification manifold, and we discuss separately in a later section. For this reason, we do not distinguish $\mathfrak{g} / \mathfrak{h}$ from its complexification.

Let us start off with the $\mathbf{1 0}$ representation and its up-type quark Yukawa coupling. The origin of $\mathbf{1 0}$ representation can be in $\mathfrak{s o}(10) / \mathfrak{s u}(5)$. If it is to be realised in Type IIA or Type IIB string theory, it is $(1,0)$ (and $(-1,0)$ ) open string connecting 5 parallel D-branes and their orientifold mirror images. It is also easy to find this representation in Type I string theory or Heterotic theory. Thus, the existence of this representation alone does not tell us a lot.

The most intriguing feature of $\mathrm{SU}(5)_{\text {GUT }}$-unified theories is that there must be up-type quark Yukawa couplings

$$
W \ni 10^{i j} 10^{k l} H(5)^{m} \epsilon_{i j k l m} .
$$

If the multiplets above were made of open strings in Type IIA or Type IIB string theories, $\mathrm{SU}(5)$-indices could be contracted only between a pair of superscript and subscript, combining a $\sigma=0$ boundary and a $\sigma=\pi$ boundary of open strings. It is unlikely that those Yukawa couplings are generated in Type IIA or Type IIB string theories. But, we know that at least in Heterotic theories, compactification of the $E_{8} \times E_{8}$ theory can provide such a Yukawa coupling, and it is also clear from the Heterotic-M-theory duality [15] and Heterotic-Ftheory duality [16, 17, 18, 19, 20, that both M-theory and F-theory can describe such Yukawa coupling, as well. Although the description will not be available within the Type IIA or IIB limits, M-theory and F-theory can describe the Yukawa couplings. We cannot expect that all the low-energy particles are described by simple open strings ending on D-branes, but by

plets have different properties under some symmetry of compactification geometry, although they are exactly in the same irreducible component in the coset space $\mathfrak{g} / \mathfrak{h}$. This possibility has to restrict the compactification geometry, instead of $\mathfrak{g} / \mathfrak{h}$ and its breaking pattern, in order to solve the problem of dimension- 4 proton decay. 
membranes 21] or by $(p, q)$ strings [22], respectively, in M-theory or in F-theory. In both descriptions, an $E_{r}$-type $(r=6,7,8)$ singularity has to be involved.

The origin of the $H(5)$ multiplet containing the up-type Higgs doublet $H_{u}$ can be identified in a coset space $\mathfrak{e}_{6} / \mathfrak{s u}(5)$, rather than $\mathfrak{s u}(N) / \mathfrak{s u}(5)$ or $\mathfrak{s o}(N) / \mathfrak{s u}(5)$ with large $N$. The irreducible decomposition ${ }^{3}$ of $\mathfrak{e}_{6}$-adj. representation is given by

$$
\begin{aligned}
\operatorname{Res}_{\mathrm{SU}(5)_{\mathrm{GUT}} \times \mathrm{SU}(2)_{2} \times \mathrm{U}(1)_{6}}^{E_{6}}\left(\mathfrak{e}_{6} \text {-adj. }\right) \simeq & (\mathbf{a d j} ., \mathbf{1})^{0} \oplus(\mathbf{1}, \mathbf{a d j} .)^{0} \oplus(\mathbf{1}, \mathbf{1})^{0} \\
& \oplus\left[\left(\wedge^{2} \mathbf{5}, \mathbf{2}\right)^{1} \oplus\left(\wedge^{4} \mathbf{5}, \wedge^{2} \mathbf{2}\right)^{2}\right] \oplus \text { h.c. },
\end{aligned}
$$

where a multiplet $\left(R_{5}, R_{2}\right)^{q_{6}}$ is in a representation $R_{5}$ of unbroken $\mathrm{SU}(5)_{\text {GUT }} \subset E_{6}$, in $R_{2}$ of underlying $\mathrm{SU}(2)_{2} \subset E_{6}$ that commutes with $\mathrm{SU}(5)$, and $q_{6}$ denotes the charge under the $\mathrm{U}(1)_{q_{6}}$ symmetry. ${ }^{4}$ This irreducible decomposition contains not only a multiplet in $\mathrm{SU}(5)_{\mathrm{GUT}^{-}}$ $\wedge^{2} \mathbf{5}$ representation, a candidate for $\mathbf{1 0}=(Q, \bar{U}, \bar{E})$ multiplets, but also one in $\mathrm{SU}(5)_{\mathrm{GUT}^{-}} \overline{\wedge^{4} \mathbf{5}}$, that is, in $\mathrm{SU}(5)_{\mathrm{GUT}^{-}} \mathbf{5}$ representation. This is a candidate of $H(\mathbf{5})$.

There is no problem in incorporating this coset space in Heterotic $E_{8} \times E_{8}$ theory, since it contains $E_{6}$ as a subgroup of $E_{8}$. The $E_{6}$ symmetry is broken down to $\mathrm{SU}(5)_{\text {GUT }}$ by turning on non-trivial vector bundle with the structure group $\mathrm{SU}(2)_{2} \times \mathrm{U}(1)_{q_{6}}$ inside the $E_{6}$. The net chirality at low energy can be obtained by the topological nature of the zero-modes of the vector bundle. In M-theory and F-theory descriptions, the $E_{6}$ vector multiplet does not come from strings, but from membranes [21] (or $(p, q)$ open strings [22]). Singular ALE fibration or elliptic fibration can give rise to $E_{6}$, which can be broken down to $\mathrm{SU}(5)_{\text {GUT }}$ by deforming the geometry in the fibre direction over base manifolds. Chiral matters arise at the loci of enhanced singularity. In any of Heterotic, M-theory and F-theory descriptions, $E_{6}$ symmetry is realised, is broken to $\mathrm{SU}(5)_{\mathrm{GUT}}$ and chiral matters in the representations in the coset space of $\mathfrak{e}_{6} / \mathfrak{s u}(5)$ are available in the low-energy effective theories. More details of the descriptions are explored in later sections. In this section, we further pursue general aspects that do not depend on the specific implementation in either one of Heterotic, M- or F-theory.

In Heterotic $E_{8} \times E_{8}$ formulation, $\mathrm{D}=10 \mathcal{N}=1$ super Yang-Mills interaction is described

\footnotetext{
${ }^{3}$ Here, $\operatorname{Res}_{H}^{G}$ means restriction of group from $G$ to $H$. This has nothing to do with residue.

${ }^{4}$ This $\mathrm{U}(1)_{q_{6}}$ symmetry and $\mathrm{U}(1)_{q_{7}}$ that appears later are related to the two $\mathrm{U}(1)$ symmetries $E_{6} \rightarrow$ $\mathrm{SO}(10) \times \mathrm{U}(1)_{\psi}$ and $\mathrm{SO}(10) \rightarrow \mathrm{SU}(5)_{\mathrm{GUT}} \times \mathrm{U}(1)_{\chi}$ in [23] as

$$
\left(\begin{array}{c}
q_{\chi} \\
q_{\psi}
\end{array}\right) \equiv\left(\begin{array}{c}
\sqrt{24} Q_{\chi} \\
\sqrt{\frac{72}{5}} Q_{\psi}
\end{array}\right)=\frac{1}{3}\left(\begin{array}{cc}
-5 & 1 \\
1 & 1
\end{array}\right)\left(\begin{array}{l}
q_{6} \\
q_{7}
\end{array}\right) .
$$


in terms of $\mathrm{D}=4$ superpotential as, e.g., [24]

$$
W=\int d^{6} y \operatorname{tr}_{E_{6}}\left(\Sigma^{a}(y, x)\left[\left(\partial^{b}-\Sigma^{b}(y, x)\right), \Sigma^{c}(y, x)\right]\right) \epsilon_{a b c}
$$

where $a=1,2,3$ correspond to 3 complex dimensions of the compact Calabi-Yau space. This comes from the second term of the Heterotic theory superpotential

$$
W \ni \int_{Z} \Omega \wedge\left(d B-\omega_{Y M}+\omega_{g r a v .}\right)
$$

where $\Omega$ is the global holomorphic 3 -form of Calabi-Yau 3-fold, and $\omega_{Y M}$ and $\omega_{\text {grav }}$. are Chern-Simons 3-forms. Chiral zero-modes live in some irreducible representations, with certain wave function depending on the internal coordinates $y$ 's. Thus, this superpotential gives rise to some interactions at low energy, and in particular, we expect that the Yukawa coupling is obtained in this way. However, since the overall compactification geometry does not preserve all the 16 SUSY charges, the value of resulting coupling constants will not be equal to the gauge coupling constant of the unbroken $\mathrm{SU}(5)_{\text {GUT }}$. It is easy to see from the Lie algebra of $E_{6}$ that the up-type quark Yukawa coupling (3) is contained in (6), when $\overline{\left(\wedge^{4} \mathbf{5}, \wedge^{2} \mathbf{2}\right)}$ is identified with $H(\mathbf{5})$ :

$$
W \ni\left(\wedge^{2} 5,2\right) \cdot\left(\wedge^{2} 5,2\right) \cdot \overline{\left(\wedge^{4} 5, \wedge^{2} 2\right)}=10 \cdot 10 \cdot H(5)
$$

The existence of the up-type quark Yukawa coupling is not specific to the Heterotic theory. The only essences are the underlying $E_{6}$ symmetry, and the existence of Yang-Mills interactions that have 16 supersymmetry charges locally. The presence or absence of interactions among chiral matters is tied to that of vector fields by the supersymmetry and is ultimately determined by the Lie algebra of $E_{6}$. Since the geometric engineering of M-theory and singular elliptic fibre of F-theory give rise to $E_{6}$ vector multiplet with 16 supersymmetry charges locally, the same results as in the Heterotic description follow in the M-theory and F-theory descriptions as well. For instance, the Figure 7 of [22] can be interpreted as the $(p, q)$ string description of the up-type quark Yukawa coupling (8).

The coset space $\mathfrak{e}_{6} / \mathfrak{s u}(5)$, however, offers only one type of multiplet $\mathrm{SU}(5)_{\mathrm{GUT}^{-}} \overline{\mathbf{5}}+$ h.c. representation. Since the minimal supersymmetric extension of the standard model contains at least two different multiplets in $\mathrm{SU}(5)_{\mathrm{GUT}} \mathbf{-} \overline{\mathbf{5}}$ representation, namely $\overline{\mathbf{5}}$ and $\bar{H}(\overline{\mathbf{5}})$, the coset space $\mathfrak{e}_{6} / \mathfrak{s u}(5)$ has to be extended so that they are incorporated. 


\subsection{The Minimal $E_{7}$ Symmetry}

A coset space $\mathfrak{e}_{7} / \mathfrak{s u}(5)$ contains $\mathfrak{e}_{6} / \mathfrak{s u}(5)$ and provides more variety in the multiplets. First, $E_{7}$ symmetry contains $\mathrm{SU}(6)_{1} \times \mathrm{SU}(2)_{2} \times \mathrm{U}(1)_{q_{6}}$, and the $\mathfrak{e}_{7}$-adj. representation has the irreducible decomposition

$$
\begin{aligned}
\operatorname{Res}_{\mathrm{SU}(6)_{1} \times \mathrm{SU}(2)_{2} \times \mathrm{U}(1)_{q_{6}}}^{E_{7}}\left(\mathfrak{e}_{7} \text {-adj. }\right) & \simeq(\mathbf{a d j} .=\mathbf{3 5}, \mathbf{1})^{0} \oplus(\mathbf{1}, \mathbf{a d j} .)^{0} \oplus(\mathbf{1}, \mathbf{1})^{0} \\
& \oplus\left[\left(\wedge^{2} \mathbf{6}, \mathbf{2}\right)^{1} \oplus\left(\wedge^{4} \mathbf{6}, \wedge^{2} \mathbf{2}\right)^{2} \oplus\left(\wedge^{6} \mathbf{6}, \mathbf{2}\right)^{3}\right] \oplus \text { h.c. }
\end{aligned}
$$

The unification group $\mathrm{SU}(5)_{\text {GUT }}$ is identified with the subgroup of $\mathrm{SU}(5) \times \mathrm{U}(1)_{q_{7}} \subset \mathrm{SU}(6)_{1}$. Thus, when the $E_{7}$ symmetry is broken down to $\mathrm{SU}(5)_{\mathrm{GUT}}$, we have the following irreducible components:

$$
\begin{aligned}
(\operatorname{adj} ., \mathbf{1})^{0} & \rightarrow(\mathbf{a d j} ., \mathbf{1})^{(0,0)} \oplus\left[(\mathbf{5}, \mathbf{1})^{(0,6)} \oplus \text { h.c. }\right] \\
\left(\wedge^{2} \mathbf{6}, \mathbf{2}\right)^{1} & \rightarrow\left[\left(\wedge^{2} \mathbf{5}, \mathbf{2}\right)^{(1,2)} \oplus(\mathbf{5}, \mathbf{2})^{(1,-4)}\right]+\text { h.c. } \\
\left(\wedge^{4} \mathbf{6}, \wedge^{2} \mathbf{2}\right)^{2} & \rightarrow\left[\left(\wedge^{4} \mathbf{5}, \wedge^{2} \mathbf{2}\right)^{(2,4)} \oplus\left(\wedge^{3} \mathbf{5}, \wedge^{2} \mathbf{2}\right)^{(2,-2)}\right]+\text { h.c. }
\end{aligned}
$$

where $\left(R_{5}, R_{2}\right)^{\left(q_{6}, q_{7}\right)}$ describe the representations under $\mathrm{SU}(5)_{\mathrm{GUT}} \times \mathrm{SU}(2)_{2} \times \mathrm{U}(1)_{q_{6}} \times \mathrm{U}(1)_{q_{7}} \subset$ $E_{7}$. In particular, there are three different multiplets in SU(5)- $\overline{5}$ representation, namely, $\overline{(\mathbf{5}, \mathbf{1})^{(0,6)}}, \overline{(\mathbf{5}, \mathbf{2})^{(1,-4)}}$ and $\left(\wedge^{4} \mathbf{5}, \wedge^{2} \mathbf{2}\right)^{(2,4)}$. Thus, the two low energy multiplets in the SU(5)-5 representation, namely, $\overline{\mathbf{5}}=(\bar{D}, L)$ and $H(\overline{\mathbf{5}})$, can be identified with 2 different multiplets. As long as they are different, there will be no dimension-4 proton decay operators (2).

It is preferable if the 2 multiplets are identified so that the down-type quark Yukawa coupling is generated from the 16-SUSY Yang-Mills interaction. In order to identify properly, let us look at the $E_{7}$ version of the superpotential (6):

$$
\begin{aligned}
W & \ni\left[\left(\wedge^{2} \mathbf{6}, \mathbf{2}\right)^{1} \cdot\left(\wedge^{2} \mathbf{6}, \mathbf{2}\right)^{1} \cdot \overline{\left(\wedge^{4} \mathbf{6}, \wedge^{2} \mathbf{2}\right)^{2}}+\left(\wedge^{2} \mathbf{6}, \mathbf{2}\right)^{1} \cdot\left(\wedge^{4} \mathbf{6}, \wedge^{2} \mathbf{2}\right)^{2} \cdot \overline{(\mathbf{1}, \mathbf{2})^{3}}\right]+\text { h.c. } \\
& +\overline{\left(\wedge^{2} \mathbf{6}, \mathbf{2}\right)^{1}} \cdot(\mathbf{a d j} . \mathbf{1})^{0} \cdot\left(\wedge^{2} \mathbf{6}, \mathbf{2}\right)^{1}+\overline{\left(\wedge^{4} \mathbf{6}, \wedge^{2} \mathbf{2}\right)^{2}} \cdot(\mathbf{a d j} ., \mathbf{1})^{0} \cdot\left(\wedge^{4} \mathbf{6}, \wedge^{2} \mathbf{2}\right)^{2}+\cdots
\end{aligned}
$$

which is, in terms of $\mathrm{SU}(5)_{\mathrm{GUT}}$,

$$
\begin{aligned}
& W \quad \ni\left(\wedge^{2} \mathbf{5}, \mathbf{2}\right)^{(1,2)} \cdot\left(\wedge^{2} \mathbf{5}, \mathbf{2}\right)^{(1,2)} \cdot \overline{\left(\wedge^{4} \mathbf{5}, \wedge^{2} \mathbf{2}\right)^{(2,4)}}+(\mathbf{5}, \mathbf{2})^{(1,-4)} \cdot\left(\wedge^{2} \mathbf{5}, \mathbf{2}\right)^{(1,2)} \cdot \overline{\left(\wedge^{3} \mathbf{5}, \wedge^{2} \mathbf{2}\right)^{(2,-2)}} \\
& +\left(\wedge^{2} \mathbf{5}, \mathbf{2}\right)^{(1,2)} \cdot\left(\wedge^{3} \mathbf{5}, \wedge^{2} \mathbf{2}\right)^{(2,-2)} \cdot \overline{(\mathbf{1}, \mathbf{2})^{(3,0)}}+(\mathbf{5}, \mathbf{2})^{(1,-4)} \cdot\left(\wedge^{4} \mathbf{5}, \wedge^{2} \mathbf{2}\right)^{(2,4)} \cdot \overline{(\mathbf{1}, \mathbf{2})^{(3,0)}} \\
& +\overline{(\mathbf{5}, \mathbf{2})^{(1,-4)}} \cdot \overline{(\mathbf{5}, \mathbf{1})^{(0,6)}} \cdot\left(\wedge^{2} \mathbf{5}, \mathbf{2}\right)^{(1,2)}+\left(\wedge^{4} \mathbf{5}, \wedge^{2} \mathbf{2}\right)^{(2,4)} \cdot \overline{(\mathbf{5}, \mathbf{1})^{(0,6)}} \cdot \overline{\left(\wedge^{3} \mathbf{5}, \wedge^{2} \mathbf{2}\right)^{(2,-2)}} \\
& + \text { h.c. }+\cdots \text {. }
\end{aligned}
$$




\begin{tabular}{|c||c|c|c|c|c|}
\hline Bundles & $\left(\wedge^{2} \mathbf{5}, \mathbf{2}\right)^{(1,2)}$ & $\overline{(\mathbf{5}, \mathbf{2})^{(1,-4)}}$ & $\overline{\left(\wedge^{4} \mathbf{5}, \wedge^{2} \mathbf{2}\right)^{(2,4)}}$ & $\overline{(\mathbf{5}, \mathbf{1})^{(0,6)}}$ & $(\mathbf{1}, \mathbf{2})^{(3,0)}$ \\
\hline Particle ID A & $(\bar{U}, Q, \bar{E})$ & $\overline{\mathbf{5}}=(\bar{D}, L)$ & $H(\mathbf{5})$ & $\bar{H}(\mathbf{5})$ & $N$ \\
Particle ID B & $(\bar{U}, Q, \bar{E})$ & $\bar{H}(\overline{\mathbf{5}})$ & $H(\mathbf{5})$ & $\overline{\mathbf{5}}=(\bar{D}, L)$ & $S$ \\
\hline
\end{tabular}

Table 1: Particle identification in $\mathfrak{e}_{7} / \mathfrak{s u}(5)$ : corresponding $\mathrm{SU}(5)_{\mathrm{GUT}} \times \mathrm{SU}(2)_{1} \times \mathrm{U}(1)_{q_{6}} \times \mathrm{U}(1)_{q_{7}}$ irreducible representations in the $\mathfrak{e}_{7}$-adj. is shown. There are 2 different possible identifications.

Therefore, for instance under the 2 identifications given in Table [ the first term provides the up-type quark Yukawa coupling (3), and the fifth term the down-type quark and chargedlepton Yukawa couplings (II). The fourth term in the hermitian-conjugate representation is interpreted as the Dirac Yukawa coupling of neutrinos ${ }^{5}$ under the identification A, and the same term is interpreted as the trilinear coupling $W=S H(\mathbf{5}) \bar{H}(\overline{\mathbf{5}})$ of some extensions of the minimal supersymmetric standard model [27, 28, 29] under the identification B.

Note that there are no dimension- 4 proton decay operators in this superpotential. It is true that (6) may not be the only interaction in the superpotential; there may be non-perturbative contribution as well. But it is inconceivable that the dimension-4 proton decay operator is generated, as we can see as follows. In either of the identification A or B, the operator (2) is an $\mathrm{SU}(2)_{2}$ doublet, not a singlet. Since $\mathrm{SU}(2)_{2}$ vector bundles in Heterotic terminology and corresponding objects in other descriptions of string theories can provide only spurions in $\mathrm{SU}(2)_{2}$-adj. representations, insertion of such spurions do not convert the doublet into a singlet. Even if we remain agnostic about the origin of corrections to the superpotential, as long as the corrections respect the underlying $E_{7}$ symmetry and its breaking is controlled by the spurions in $\mathrm{SU}(2)_{2} \times \mathrm{U}(1)_{q_{6}} \times \mathrm{U}(1)_{q_{7}}$, the dimension-4 proton decay operators are forbidden by the $E_{7}$ symmetry. The absence of dimension-5 proton decay operators

$$
W \ni 10.10 .10 . \overline{5}
$$

also follow from the same argument in the case of the identification B. In the case of identification $\mathrm{A}$, these operators can be $\mathrm{SU}(2)_{2}$ singlet, and only $\mathrm{U}(1)$ charges do not vanish.

\footnotetext{
${ }^{5}$ Note that right-handed neutrinos (in the identification A in Table 1 naturally arises in the spectrum, although we did not necessarily require their presence. Indeed, the existence of right-handed neutrinos does not necessarily favours $\mathrm{SO}(10)$ unification; the spinor representation of $\mathrm{SO}(10)$ certainly contains a righthanded neutrino, but it remains as a part of moduli (vector bundle moduli in Heterotic theory, and complex structure moduli in F-theory) in string theory terminology when $\mathrm{SU}(5)$ unified theory is considered instead of $\mathrm{SO}(10)$ theories. This is almost clear from the discussion in [25] 26]. See also a recent article [8].
} 
Since U(1) symmetry may have mixed anomalies, Green-Schwarz fields may effectively supply the $\mathrm{U}(1)$ charges. Thus, it is not absolutely clear that the dimension-5 proton decay operators are absent in the presence of non-perturbative corrections. But, on the other hand, it is worthwhile to note that a reasonable exponential suppression in non-perturbative effects is sufficient to make it consistent with the experimental limits, since the limits on the dimension- 5 operators are not that stringent as those on the dimension- 4 operators.

Thus, we have seen that the $E_{7}$ symmetry (or 2-cycles with $E_{7}$ intersection form) is minimal when we require all the Yukawa couplings from the Yang-Mills interaction, and different origins for $\overline{\mathbf{5}}$ and $\bar{H}(\overline{\mathbf{5}})$ in order to avoid the dimension-4 proton decay. It is not difficult to realise the $E_{7}$ symmetry and its breaking to $\mathrm{SU}(5)_{\mathrm{GUT}}$ in any branches of string theories, just as in the case of $E_{6}$. For instance, the Figure 8 of [22] can be regarded as $(p, q)$ string picture of the Dirac Yukawa coupling of neutrinos in the identification A, or the $W \ni$ $S H \bar{H}$ interaction in the identification B. Vector bundles (in Heterotic terminology) that break the $E_{7}$ symmetry down to $\mathrm{SU}(5)_{\text {GUT }}$ have to maintain the distinction between $(\mathbf{5}, \mathbf{2})^{(1,-4)}$ and $(\mathbf{5}, \mathbf{1})^{(0,6)}$. Thus, the bundle is supposed to have the structure group $\mathrm{SU}(2)_{2} \times \mathrm{U}(1)_{q_{6}} \times \mathrm{U}(1)_{q_{7}}$.

\subsection{Extension to the $E_{8}$ Symmetry}

The observation that the $E_{7}$ is the minimal underlying symmetry implies that the $E_{8}$ symmetry of the Heterotic theory is not strictly necessary in order to construct a "realistic" 6 model. But all Heterotic, D=11 SUGRA and F-theory admit up to the $E_{8}$ symmetry, and it is also interesting as an alternative to think of identification in $\mathfrak{e}_{8} / \mathfrak{s u}(5)$. This can be done simply by embedding the identification in the minimal $\mathfrak{e}_{7} / \mathfrak{s u}(5)$ coset through

$$
\begin{gathered}
E_{7} \times \mathrm{SU}(2)_{1} \subset E_{8}, \quad \text { or } \quad \mathfrak{e}_{7}+\mathfrak{s u}(2)_{1} \subset \mathfrak{e}_{8} . \\
\operatorname{Res}_{E_{7} \times \mathrm{SU}(2)_{1}}^{E_{8}}\left(\mathfrak{e}_{8} \text {-adj. }\right) \simeq(\mathbf{a d j} ., \mathbf{1}) \oplus(\mathbf{1}, \mathbf{a d j} .) \oplus(\mathbf{5 6}, \mathbf{2}) .
\end{gathered}
$$

Since we have already seen the irreducible decomposition of $\mathfrak{e}_{7}$-adj., we are left with $(\mathbf{5 6}, \mathbf{2})$, which decomposes into

$$
\operatorname{Res}_{\mathrm{SU}(6)_{1} \times \mathrm{SU}(2)_{1} \times \mathrm{SU}(2)_{2} \times \mathrm{U}(1)_{q_{6}}}^{E_{7} \times \mathrm{SU}()_{1}}(\mathbf{5}, \mathbf{2}) \simeq\left[(\mathbf{6}, \mathbf{2}, \mathbf{2})^{-1} \oplus(\mathbf{6}, \mathbf{2}, \mathbf{1})^{2}\right] \oplus \text { h.c. } \oplus\left(\wedge^{3} \mathbf{6}, \mathbf{2}, \mathbf{1}\right)^{0} .
$$

\footnotetext{
${ }^{6}$ Note, however, that we have not considered how the $\mathrm{SU}(5)_{\text {GUT }}$ symmetry is broken down to the standard model gauge group. See below.
} 
After further decomposing into $\mathrm{SU}(5)_{\mathrm{GUT}}$-irreducible pieces, one can see that the $\mathfrak{e}_{8}$-adj. representation provides three multiplets in $\mathrm{SU}(5)_{\mathrm{GUT}^{-}} \wedge^{2} \mathbf{5}$ representation

$$
\left(\wedge^{2} \mathbf{1 0}, \mathbf{1} \oplus \mathbf{2}_{1} \oplus \mathbf{2}_{2}\right)=\left(\begin{array}{r}
\left(\wedge^{2} \mathbf{5}, \mathbf{1}, \mathbf{1}\right)^{(-2,2)} \\
\left(\wedge^{2} \mathbf{5}, \mathbf{2}, \mathbf{1}\right)^{(0,-3)} \\
\mathbf{1 0}=\left(\wedge^{2} \mathbf{5}, \mathbf{1}, \mathbf{2}\right)^{(1,2)}
\end{array}\right)
$$

where $\left(R_{5}, R_{1}, R_{2}\right)^{\left(q_{6}, q_{7}\right)}$ on the right-hand side denotes the representation under the $\mathrm{SU}(5)_{\mathrm{GUT}} \times$ $\mathrm{SU}(2)_{1} \times \mathrm{SU}(2)_{2}$ and the charge under $\mathrm{U}(1)_{q_{6}}$ and $\mathrm{U}(1)_{q_{7}}$. There are 5 multiplets in the $\mathrm{SU}(5)_{\mathrm{GUT}^{-}} \overline{\mathbf{5}}$ representation

$$
\left(\overline{\mathbf{5}}, \wedge^{2}\left(\mathbf{1} \oplus \mathbf{2}_{1} \oplus \mathbf{2}_{2}\right)\right) \simeq\left(\begin{array}{r|r}
(\overline{\mathbf{5}}, \mathbf{2}, \mathbf{1})^{(-2,-1)} & \overline{\mathbf{5}}=(\overline{\mathbf{5}}, \mathbf{1}, \mathbf{2})^{(-1,4)} \\
\bar{H}(\overline{\mathbf{5}})=(\overline{\mathbf{5}}, \mathbf{1}, \mathbf{1})^{(0,-6)} & (\overline{\mathbf{5}}, \mathbf{2}, \mathbf{2})^{(1,-1)} \\
\hline & H(\mathbf{5})^{\dagger}=(\overline{\mathbf{5}}, \mathbf{1}, \mathbf{1})^{(2,4)}
\end{array}\right)
$$

and there are $3 \mathrm{SU}(5)_{\mathrm{GUT}}$-singlets

$$
\left(\mathbf{1}, \mathbf{a d j} .\left(\mathbf{1} \oplus \mathbf{2}_{1} \oplus \mathbf{2}_{2}\right)\right) \simeq\left(\begin{array}{ll|l} 
& (\mathbf{1}, \mathbf{2}, \mathbf{1})^{(-2,5)} & \\
\hline N=(\mathbf{1}, \mathbf{1}, \mathbf{2})^{(3,0)} & (\mathbf{1}, \mathbf{2}, \mathbf{2})^{(1,5)} &
\end{array}\right) .
$$

Corresponding low-energy multiplets are also noted above, following the identification A in Table 1 in $\mathfrak{e}_{7} / \mathfrak{s u}(5)$ and the embedding (16). The irreducible decompositions (19)-(21) are a special cases of $\mathrm{SU}(5)$-bundle compactification of the Heterotic $E_{8} \times E_{8}$ theory; the rank-5 $\mathrm{SU}(5)$ vector bundle $V_{5}$ is now split up into

$$
V_{5} \simeq \mathbf{1} \oplus \mathbf{2}_{1} \oplus \mathbf{2}_{2}
$$

with the structure group reduced to $\mathrm{SU}(2)_{1} \times \mathrm{SU}(2)_{2} \times \mathrm{U}(1)_{q_{6}} \times \mathrm{U}(1)_{q_{7}}$. The singlets in (21) are would-be vector bundle moduli if the structure group were $\mathrm{SU}(5)$.

Since we only need to maintain the different origins for $\overline{\mathbf{5}}$ and $\bar{H}(\mathbf{5})$ multiplets, the $\mathfrak{e}_{8} / \mathfrak{s u}(5)$ coset does not have to be split up as much as in (19)-(21). In particular, the vector bundles $V_{5}=\mathbf{1} \oplus \mathbf{2}_{1} \oplus \mathbf{2}_{2}$ can be a little more generic. Let us use the particle identification A in Table 1inside (19)-(21) for concreteness ${ }^{7}$ in the following discussion. Then, the distinction between the 2 multiplets in $\overline{\mathbf{5}}$ representation is not lost when the $\mathrm{SU}(2)_{1} \times \mathrm{U}(1)_{\tilde{q}_{6}}$ bundle $^{8} \mathbf{1} \oplus \mathbf{2}_{1}$ is

\footnotetext{
${ }^{7}$ The following discussion does not change essentially when the ID B is adopted.

${ }^{8}$ The U(1) symmetries of $\tilde{q}_{6}$ and $\tilde{q}_{7}$ are different linear combinations of $q_{6}$ and $q_{7}$ :

$$
\left(\begin{array}{c}
\tilde{q}_{6} \\
\tilde{q}_{7}
\end{array}\right)=\frac{1}{3}\left(\begin{array}{cc}
2 & -1 \\
-5 & -2
\end{array}\right)\left(\begin{array}{l}
q_{6} \\
q_{7}
\end{array}\right), \quad\left(\begin{array}{l}
q_{6} \\
q_{7}
\end{array}\right)=\frac{1}{3}\left(\begin{array}{cc}
2 & -1 \\
-5 & -2
\end{array}\right)\left(\begin{array}{l}
\tilde{q}_{6} \\
\tilde{q}_{7}
\end{array}\right) .
$$
}


replaced by a rank-3 SU(3) bundle $\mathbf{3}_{2}$, so that the rank- 5 vector bundle is $V_{5}=\mathbf{3}_{2} \oplus \mathbf{2}_{2}$ with the structure group $\mathrm{SU}(3)_{2} \times \mathrm{SU}(2)_{2} \times \mathrm{U}(1)_{\tilde{q}_{7}}$ :

$$
\begin{aligned}
\left(\mathbf{1 0}, \mathbf{3}_{2} \oplus \mathbf{2}_{2}\right) & \simeq\left(\begin{array}{c}
\left(\mathbf{1 0}, \mathbf{3}_{2}\right) \\
\hline \mathbf{1 0}=\left(\mathbf{1 0}, \mathbf{2}_{2}\right)
\end{array}\right) \\
\left(\overline{\mathbf{5}}, \wedge^{2}\left(\mathbf{3}_{2} \oplus \mathbf{2}_{2}\right)\right) & \simeq\left(\begin{array}{l|l}
\bar{H}(\overline{\mathbf{5}})=\left(\overline{\mathbf{5}}, \wedge^{2} \mathbf{3}_{2}\right) & \overline{\mathbf{5}}=\left(\overline{\mathbf{5}}, \mathbf{3}_{2} \otimes \mathbf{2}_{2}\right) \\
\hline & H(\mathbf{5})^{\dagger}=\left(\overline{\mathbf{5}}, \wedge^{2} \mathbf{2}_{2}\right)
\end{array}\right) .
\end{aligned}
$$

Likewise, the bundle $\mathbf{2}_{1} \oplus \mathbf{2}_{2}$ can be replaced by a rank- 4 vector bundle $\mathbf{4}$ with the structure group $\mathrm{SU}(4) \supset \mathrm{SU}(2)_{1} \times \mathrm{SU}(2)_{2} \times \mathrm{U}(1)_{\psi}$, so that the rank-5 bundle becomes $V_{5}=\mathbf{1} \oplus \mathbf{4}$ with the structure group $\mathrm{U}(1)_{\chi} \times \mathrm{SU}(4)$. The reduced rank-5 bundles $V_{5}=\mathbf{3}_{2} \oplus \mathbf{2}_{2}$, and $V_{5}=\mathbf{1} \oplus \mathbf{4}$ are much more generic than $V_{5}=\mathbf{1} \oplus \mathbf{2}_{1} \oplus \mathbf{2}_{2}$, yet the original motivation is not lostdistinguishing $\overline{\mathbf{5}}$ and $\bar{H}(\overline{\mathbf{5}})$ in their origins.

The important difference between the two different choice of vector bundles is that $H(\mathbf{5})$ and $\bar{H}(\overline{\mathbf{5}})$ are in a pair of hermitian-conjugate representation when $V_{5}=\mathbf{1} \oplus \mathbf{4}$, but they are not when $V_{5}=\mathbf{3}_{2} \oplus \mathbf{2}_{2}$. Indeed, in $\mathfrak{e}_{7} / \mathfrak{s u}(5)$, they cannot be identified with a vector-like pair when all the Yukawa coupling are required to be generated. But under the embedding $\mathfrak{e}_{7} / \mathfrak{s u}(5)$ into $\mathfrak{e}_{8} / \mathfrak{s u}(5)$ through (16) , the $\mathrm{SU}(2)_{1}$-singlet $\bar{H}(\overline{\mathbf{5}})$ is regarded as $\left(\overline{\mathbf{5}}, \wedge^{2} \mathbf{2}_{1}, \mathbf{1}\right)$ representation of $\mathrm{SU}(5)_{\mathrm{GUT}} \times \mathrm{SU}(2)_{1} \times \mathrm{SU}(2)_{2}$; since $H(\mathbf{5})$ is in $\overline{\left(\overline{\mathbf{5}}, \mathbf{1}, \wedge^{2} \mathbf{2}_{2}\right)}$, both $H(\mathbf{5})$ and $\bar{H}(\overline{\mathbf{5}})$ are from the same (up to conjugation) multiplet $\left(\overline{\mathbf{5}}, \wedge^{2} \mathbf{4}\right)$ when the 2 rank-2 bundles $\mathbf{2}_{1}$ and $\mathbf{2}_{2}$ mix up into an irreducible $\mathrm{SU}(4)$ bundle 4.

If the doublet-triplet splitting problem is to be solved by a Wilson line [30], they have to be in a Hermitian-conjugate pair. A Wilson line does not make a difference in the Euler characteristics for triplets and doublets. Thus, the Wilson line as the solution to the problem motivates to promote $\mathfrak{e}_{7} / \mathfrak{s u}(5)_{\text {GUT }}$ to $\mathfrak{e}_{8} / \mathfrak{s u}(5)_{\text {GUT }}$ for the origin of low energy particles. Incidentally, $E_{8}$ is a maximal symmetry or singularity available in string theories. However, the missing-partner type mass matrix of the Higgs sector [31, ${ }^{9}$ another potential solution to In the $\mathrm{SU}(5)$ structure group of $V_{5}$, the $\mathrm{U}(1)$ generators are

$$
\begin{aligned}
\mathbf{q}_{6}=\operatorname{diag}(-2,0,0,1,1), & \mathbf{q}_{7}=\operatorname{diag}(2,-3,-3,2,2), \\
\mathbf{q}_{\chi}=\operatorname{diag}(4,-1,-1,-1,-1), & \mathbf{q}_{\psi}=\operatorname{diag}(0,-1,-1,1,1), \\
\tilde{\mathbf{q}}_{6}=\operatorname{diag}(-2,1,1,0,0), & \tilde{\mathbf{q}}_{7}=\operatorname{diag}(2,2,2,-3,-3),
\end{aligned}
$$

\footnotetext{
${ }^{9}$ There is a variety in the $\mathrm{D}=4$ field-theory realization of the missing partner mechanism, and the matter contents do not have to be the original one. There will be more variety in implementing the missing partner mechanism in string theories. Reference [32] is an attempt to embed a field-theory model 33 into Type IIB string theory, and is one of such variety.
} 
the doublet-triplet splitting problem, does not necessarily require that they originate from a pair of conjugate representation.

In the next section, the idea of having reducible vector bundles (in Heterotic terminology) as a solution to the dimension-4 proton decay problem is implemented in Heterotic theory description. In later sections, M-theory and F-theory descriptions are given.

\section{Heterotic Vacua}

The Yukawa couplings have been discussed in the several previous approaches to Heterotic string compactification [2, 7, 4, 8]. The novelty in our story is that we consider simultaneously the Yukawa couplings and proton decay terms. In our consideration, we show that the presence of the former and absence of the latter appears naturally, thus providing a Heterotic picture of the discussion in the previous section. In the subsequent sections, we will provide M-theory and F-theory pictures for the same field theory constrains.

In compactification of Heterotic theory, in order to have $\mathrm{D}=4$ effective field theories with unbroken $\mathrm{SU}(5)_{\text {GUT }}$ symmetry, one has only to turn on an $\mathrm{SU}(5)$ vector bundle in one of $E_{8}$ gauge group of $E_{8} \times E_{8}$. The absence of dimension- 4 proton decay operators in our vacuum suggests, however, that there will be more structure than that. Here we work on a possibility that the $\overline{\mathbf{5}}=(\bar{D}, L)$ and $\bar{H}(\overline{\mathbf{5}})$ have different origins in the $\mathfrak{e}_{8}$-adj. representation, so that the down-type quark Yukawa couplings exist whereas the dimension-4 proton decay operators do not. This idea is implemented when the rank-5 SU(5) vector bundle on a Calabi-Yau 3-fold $Z$ becomes reducible and is split up into two irreducible vector bundles ${ }^{10}$

$$
V_{5}=L \oplus U_{4}, \quad \text { with } \quad L \otimes \operatorname{det} U_{4} \simeq \mathcal{O}_{Z}
$$

where $U_{4}$ and $L$ are rank-4 and rank-1 vector bundles, respectively, with the structure group $\mathrm{U}(4)$ and $\mathrm{U}(1)$. An alternative is

$$
V_{5}=U_{3} \oplus U_{2}, \quad \text { with } \quad \operatorname{det} U_{3} \otimes \operatorname{det} U_{2} \simeq \mathcal{O}_{Z}
$$

where $U_{3}$ and $U_{2}$ are rank-3 and rank-2 vector bundles with structure group $\mathrm{U}(3)$ and $\mathrm{U}(2)$, respectively. This can be regarded as the particular choice of vector-bundle moduli parameter of $\mathrm{SU}(5)$ vector bundles in [34]. The $2+2+1$ reducible vector bundle (22) corresponds to a

\footnotetext{
${ }^{10}$ We start our discussion with a direct sum splitting of the tank- 5 vector bundle. Later we will consider the case when some neutrino fields acquire expectation values and the rank- 5 bundle becomes an extension of $U_{4}$ by L.
} 


\begin{tabular}{|c||c|c|c|c|c|c|c|} 
bundles & $U_{4}$ & $U_{4} \otimes L$ & $\overline{\wedge^{2} U_{4}}$ & $\wedge^{2} U_{4}$ & $U_{4} \otimes L^{-1}$ & $\mathbf{1}$ & $L$ \\
\hline low-energy particles & $(Q, \bar{U}, \bar{D})$ & $(\bar{D}, L)$ & $H$ & $\bar{H}$ & $\bar{N}$ & $((\mathbf{a d j} \cdot))$ & $\left(\left(\mathbf{1 0}^{\prime}\right)\right)$
\end{tabular}

Table 2: Particle identification in $U_{4} \oplus L$ bundle compactification of Heterotic $E_{8} \times E_{8}$ theory. The last two columns mean that particles from the 2 bundles are not necessary in our lowenergy effective theory.

particular choice of moduli parameter of (29) and also of (30). The choice of the bundle (29) reduces to [4] when the line bundle $L$ is a flat bundle. The reducible bundle (29) was originally considered in 35 .

Although the different bundles have different physical consequences, the string-theoretical study for each bundles is qualitatively the same. Thus, we discuss the case of (29) extensively and only mention the differences in the cases of (30) and (22) bundles later.

\subsection{Compactification with $4+1$ Vector Bundles}

\subsubsection{Spectrum and Yukawa Couplings}

The low-energy multiplets in $\mathrm{SU}(5)_{\text {GUT }} \mathbf{- 1 0}$ representation arise from zero-modes (cohomology) of the vector bundle $V_{5}$, which is now split up into 2 irreducible pieces $U_{4}$ and $L$. Those in $\mathrm{SU}(5)_{\mathrm{GUT}}-\overline{\mathbf{5}}$ (or $\mathbf{5}$ ) representation arise from $\wedge^{2} V_{5}$, which now consists of 2 irreducible components $\wedge^{2} U_{4}$ and $U_{4} \otimes L$ (or bundles in the conjugate representations). Finally, the $\mathrm{SU}(5)$ vector bundle moduli from adj. $\left(V_{5}\right)$ - which are $\mathrm{SU}(5)_{\mathrm{GUT}}$-singlets - are split up into 2 pieces, namely the $\mathrm{U}(4)$ vector bundle moduli and $\mathrm{SU}(5)_{\mathrm{GUT}}$-singlets from the bundle $U_{4} \otimes L^{-1}$. When the low-energy multiplets are from the bundles specified in Table 2, all the Yukawa coupling are generated from the $E_{8}$ Yang-Mills interaction, including the Dirac Yukawa coupling of neutrinos:

$$
\begin{aligned}
W \ni & \left(\mathbf{1 0}, U_{4}\right) \otimes\left(\mathbf{1 0}, U_{4}\right) \otimes \overline{\left(\overline{\mathbf{5}}, \wedge^{2} U_{4}\right)} \longrightarrow \mathbf{1 0 . 1 0 . H ( 5 )} \\
& +\left(\overline{\mathbf{5}}, U_{4} \otimes L\right) \otimes\left(\mathbf{1 0}, U_{4}\right) \otimes\left(\overline{\mathbf{5}}, \wedge^{2} U_{4}\right) \longrightarrow \overline{\mathbf{5}} \cdot \mathbf{1 0} \cdot \bar{H}(\overline{\mathbf{5}}) \\
& +\left(\overline{\mathbf{5}}, U_{4} \otimes L\right) \otimes\left(\mathbf{1}, U_{4} \otimes L^{-1}\right) \otimes \overline{\left(\overline{\mathbf{5}}, \wedge^{2} U_{4}\right)} \longrightarrow \overline{\mathbf{5}} \cdot \bar{N} \cdot H(\mathbf{5})
\end{aligned}
$$

On the other hand, the dimension 4 proton decay operator (2) is not generated, because

$$
\left(U_{4} \otimes L\right) \otimes U_{4} \otimes\left(U_{4} \otimes L\right)
$$

does not contain a trivial representation in its irreducible decomposition. The operator (2)

is certainly invariant under $\mathrm{SU}(5)_{\text {GUT }}$, but not under full $E_{8}$. The symmetry $\mathrm{U}(1)_{\chi}$ (or 
equivalently $\mathrm{U}(1)_{\mathrm{B}-\mathrm{L}}$ ) and the bundle structure group $\mathrm{SU}(4)$ guarantees the absence of the dangerous operators.

Note also that all of $\mathbf{1 0}, \overline{\mathbf{5}}$ and $\bar{N}$ would come from the same bundle $U_{4}$ if the line bundle $L$ were trivial (or flat). This was the situation discussed in $\mathrm{SO}(10)$ models of [4]. The line bundle $L \simeq \operatorname{det} U_{4}^{-1}$ is in the direction of $\mathrm{U}(1)_{\chi}$.

It is straightforward to obtain the formulae for the net chirality of low-energy multiplets. Let us take a Calabi-Yau 3-fold $Z$ and a vector bundle $V$ on it. The $\mathfrak{e}_{8}$-adj. representation is split up into irreducible pieces under the the unbroken $\mathrm{SU}(5)_{\text {GUT }}$ and the structure group of the vector bundle. The number of chiral multiplets in the $\left(R_{5}, \rho(V)\right)$ representation is given by $H^{1}(Z, \rho(V))$, and that of multiplets in the conjugate representation by $H^{1}(Z, \bar{\rho}(V))$. The net chirality at low energy is given by

$$
\begin{aligned}
\chi\left(R_{5}\right) & \equiv \#\left(R_{5}, \rho(V)\right)-\#\left(\overline{R_{5}}, \bar{\rho}(V)\right), \\
& =h^{1}(Z ; \rho(V))-h^{1}(Z ; \overline{\rho(V)}), \\
& =-\chi(Z, \rho(V))=-\int_{Z} \operatorname{ch}(\rho(V)) \operatorname{Td}(T Z),
\end{aligned}
$$

where the last equality is the Hirzebruch-Riemann-Roch theorem [36]. In an application to the bundle of interest, ${ }^{11}$

$$
\begin{aligned}
\chi\left(Z, U_{4}\right) & =-\frac{1}{12} c_{2}(T Z) c_{1}(L)-\frac{1}{6} c_{1}(L)^{3}+\frac{1}{2} c_{2}\left(U_{4}\right) c_{1}(L)+\frac{1}{2} c_{3}\left(U_{4}\right), \\
\chi(Z, L) & =+\frac{1}{12} c_{2}(T Z) c_{1}(L)+\frac{1}{6} c_{1}(L)^{3}, \\
\chi\left(Z, U_{4} \otimes L\right) & =+\frac{1}{4} c_{2}(T Z) c_{1}(L)+\frac{1}{2} c_{1}(L)^{3}-\frac{1}{2} c_{2}\left(U_{4}\right) c_{1}(L)+\frac{1}{2} c_{3}\left(U_{4}\right), \\
\chi\left(Z, \wedge^{2} U_{4}\right) & =-\frac{1}{4} c_{2}(T Z) c_{1}(L)-\frac{1}{2} c_{1}(L)^{3}+c_{2}\left(U_{4}\right) c_{1}(L) \\
\chi\left(Z, U_{4} \otimes L^{-1}\right) & =-\frac{5}{12} c_{2}(T Z) c_{1}(L)-\frac{11}{6} c_{1}(L)^{3}+\frac{3}{2} c_{2}\left(U_{4}\right) c_{1}(L)+\frac{1}{2} c_{3}\left(U_{4}\right) .
\end{aligned}
$$

The Calabi-Yau condition $c_{1}(T Z)=0$ was also used in the above calculation. Note that the net chirality for the multiplets in $\mathrm{SU}(5)_{\mathrm{GUT}^{-}} \mathbf{1 0}$ representation is the same as that for those in $\mathrm{SU}(5)_{\mathrm{GUT}^{-}} \overline{\mathbf{5}}$ representation:

$$
(\underline{34})+(35)=(36)+(37)=\frac{1}{2} \int_{Z} c_{3}\left(U_{4}\right)+\frac{1}{2} \int_{Z} c_{2}\left(U_{4}\right) c_{1}(L)=\frac{1}{2} \int_{Z} c_{3}\left(V_{5}\right) .
$$

This serves as a good check of the above calculation [35].

\footnotetext{
${ }^{11}$ Integration over $Z$ is omitted, just for visual clarity.
} 
Since we do not want a non-zero net chirality from the Higgs sector, our vacuum should be based on

$$
\chi\left(Z, \wedge^{2} U_{4}\right)=0 .
$$

Since $\mathrm{SU}(5)_{\mathrm{GUT}}-\mathbf{1 0} \mathbf{0}^{\prime}$ multiplets from the bundle $L$ do not have appropriate Yukawa couplings, they cannot have mass terms. Since we have not seen exactly massless $\mathrm{SU}(5)_{\mathrm{GUT}}$-charged particles, there should not be non-zero net chirality for this multiplet which tells us that:

$$
\chi\left(\mathbf{1 0}^{\prime}\right)=-\chi(Z, L)=0 .
$$

Combining these phenomenological inputs (40) and (41) with general chirality formulae (35) and (37), we see that our vacuum has to satisfy

$$
\begin{aligned}
\int_{Z} c_{2}(T Z) c_{1}(L) & =-2 \int_{Z} c_{1}(L)^{3}, \\
\int_{Z} c_{2}\left(U_{4}\right) c_{1}(L) & =0 .
\end{aligned}
$$

Substituting these two relations back into (34), (36) and (38), the number of generations is given by

$$
\begin{aligned}
N_{\text {gen }}=\chi(\mathbf{1 0})=\chi(\overline{\mathbf{5}}) & =-\int_{Z} \frac{1}{2} c_{3}\left(U_{4}\right), \\
\chi(\bar{N}) & =-\int_{Z} \frac{1}{2} c_{3}\left(U_{4}\right)+\int_{Z} c_{1}(L)^{3} .
\end{aligned}
$$

The "chirality" of right-handed neutrinos (45) is no longer equal to that of other quarks and leptons, but there is nothing wrong phenomenologically. Since the $\mathrm{SO}(10)$ or $\mathrm{U}(1)_{\chi}$ gauge symmetry is already broken, there is no reason why exactly 3 copies of right-handed neutrinos exist. Note also that (45) represents the minimal number of right-handed neutrinos.

The $\mathrm{SU}(5)_{\text {GUT }}$ symmetry may be broken by introducing a Wilson line. If there is a discrete symmetry group $\Gamma$ mapping $Z$ to itself without a fixed point, $Z / \Gamma$ can be used for compactification instead of $Z$. The Wilson line can be introduced on the non-trivial $\pi_{1}(Z / \Gamma)$. In this case, the number of generation of quarks and leptons are given by

$$
N_{\text {gen }}^{\prime}=-\frac{1}{\# \Gamma} \frac{1}{2} \int_{Z} c_{3}\left(U_{4}\right) \text {. }
$$

This is supposed to be 3 for our vacuum. \# $\Gamma$ should divide $-\int_{Z} c_{1}(L)^{3}=\int_{Z} c_{1}\left(U_{4}\right)^{3}$. 


\subsubsection{Anomalous $\mathrm{U}(1)_{\chi}$ Symmetry and Instability}

As we saw in (45), the "chirality" of right-handed neutrinos is generically different from that of quarks and leptons when $c_{1}(L) \neq 0$, indicating that the $\mathrm{U}(1)_{\chi}$ symmetry is anomalous. All the necessary Green-Schwarz couplings are worked out in [35] where a detailed account of the conventions can be found. One can see there that various triangle anomalies involving this symmetry are cancelled by the shift of the imaginary part of dilaton and Kähler moduli chiral multiplets, $S$ and $T^{k}$, respectively. All of them come from the $B$ field. Certain linear combination of the imaginary parts are eaten as the longitudinal mode of the anomalous $\mathrm{U}(1)_{\chi}$ vector boson. The scalar-vector mixing interactions arise from the Kähler potential

$$
K=-M_{G}^{2}\left(\ln \left(\frac{1}{3 !} \int \tilde{J} \wedge \tilde{J} \wedge \tilde{J}\right)+\ln \left(S+S^{\dagger}-Q^{0} V\right)\right),
$$

where $M_{G} \simeq 2.4 \times 10^{18} \mathrm{GeV}, S$ is the dilaton chiral multiplet whose expectation value is $1 / g_{\mathrm{YM}}^{2}$ of $\mathrm{SU}(5)_{\mathrm{GUT}}$ in the absence of 1-loop corrections, and

$$
\frac{1}{2 \pi}(-J+i B)=l_{s}^{2} T^{k} \omega_{k}, \quad \tilde{J}=-\pi l_{s}^{2}\left(T^{k}+T^{k \dagger}-Q^{k} V\right) \omega_{k},
$$

where $J$ is the Kähler form of the compactification manifold $Z, \omega_{k}\left(k=1, \cdots, h^{1,1}(Z)\right)$ are integral basis of the second cohomology of $Z, l_{s}=2 \pi \sqrt{\alpha^{\prime}}$ and $T^{k}$ are chiral multiplets containing the $\omega_{k}$-component of the Kähler modulus and the $B$ field. Under the U(1) symmetry,the charged chiral multiplets $\Psi_{i}$ such as $\bar{N}, \mathbf{1 0}$ and $\overline{\mathbf{5}}$ and the moduli chiral multiplets transform as

$$
\begin{aligned}
V & \rightarrow V-i \Lambda+i \Lambda^{\dagger}, \\
\Psi_{i} & \rightarrow e^{i q_{i} \Lambda} \Psi_{i} \\
S & \rightarrow S-i Q^{0} \Lambda, \\
T^{k} & \rightarrow T^{k}-i Q^{k} \Lambda,
\end{aligned}
$$

with $^{12}$

$$
\begin{aligned}
Q^{0} & =-\frac{2 \operatorname{tr}_{f}\left(\mathbf{q q}_{L}\right)}{32 \pi^{2}} \int_{Z} c_{1}(L)\left(c_{2}\left(V_{5}\right)-\frac{1}{2} c_{2}(T Z)\right), \\
Q^{k} & =-\frac{2 \operatorname{tr}_{f}\left(\mathbf{q q}_{L}\right)}{8 \pi^{2}} q_{L}^{k}, \\
c_{1}(L) & \equiv q_{L}^{k} \omega_{k} .
\end{aligned}
$$

\footnotetext{
${ }^{12}$ The charges adopted below differ from those in 35 by a factor of $(2 \pi)$. Coefficients in this article are corrected in version 2, following [37.
} 
The trace is taken in the fundamental representation of the bundle group $\mathrm{SU}(5)$. In the case of the $\mathrm{U}(1)_{\chi}$ anomalous symmetry, $\mathbf{q}_{L}=\operatorname{diag}(1,-1 / 4,-1 / 4,-1 / 4,-1 / 4)$, and we can choose the normalisation of the charges of $\mathrm{U}(1)_{\chi}$ symmetry as $\mathbf{q}=\mathbf{q}_{\chi} \equiv \operatorname{diag}(4,-1,-1,-1,-1)$ for convenience. ${ }^{13}$ With this choice of $\mathbf{q}=\mathbf{q}_{\chi}$, the 10-representations from $U_{4}$ bundle have -1 unit charge, and $\bar{N}$ from $U_{4} \otimes L^{-1}$ bundle have -5 units. If $h^{1}\left(Z ; \bar{U}_{4} \otimes L\right) \neq 0$, so that there is an anti-generation right-handed neutrino $\bar{N}$ in the spectrum, its $\mathrm{U}(1)_{\chi}$ charge is 5 units.

The Fayet-Iliopoulos parameter of such anomalous U(1) symmetries can be obtained by ${ }^{14}$ $\mathcal{L} \ni D \xi=\left.\left.V\right|_{\theta^{2} \bar{\theta}^{2}}(\partial K / \partial V)\right|_{V=0}$, and in the case of $\mathrm{U}(1)_{\chi}$,

$$
\xi_{\chi}=\frac{10 M_{G}^{2}}{32 \pi^{2}}\left[\frac{2 \pi l_{s}^{2}}{\operatorname{vol}(Z)} \int c_{1}(L) \wedge J \wedge J-\frac{g_{\mathrm{YM}}^{2} e^{2 \widetilde{\phi}_{4}}}{2} \int c_{1}(L)\left(c_{2}\left(V_{5}\right)-\frac{1}{2} c_{2}(T Z)\right)\right] .
$$

The first term is the tree level Fayet-Iliopoulos parameter, which comes from the derivative with respect to $T^{k}$, and depends on Kähler moduli $T^{k}$ 's through the Kähler form $J$. The second term is the 1-loop correction, and depends on the fluctuation of the dilaton $e^{\widetilde{\phi}_{4}}$. This Fayet-Iliopoulos parameter is used as in

$$
\mathcal{L}=\frac{1}{2 g_{\mathrm{YM}}^{2}} 2 \operatorname{tr}{ }_{f}\left(\mathbf{q}_{\chi}^{2}\right) D_{\chi}^{2}+D_{\chi} \xi_{\chi}+D_{\chi} q_{\chi, i} \psi_{i}^{\dagger} \psi_{i} \rightarrow V=\frac{1}{2} \frac{g_{\mathrm{YM}}^{2}}{2 \operatorname{tr}_{f}\left(\mathbf{q}_{\chi}^{2}\right)}\left(\xi_{\chi}+q_{\chi, i} \psi_{i}^{\dagger} \psi_{i}\right)^{2}
$$

The equations of motions of Yang-Mills fields of the vector bundles $L$ and $U_{4}$ are given by 38

$$
2 g^{\bar{\beta} \alpha} \frac{F_{\alpha \bar{\beta}}^{\left(L, U_{4}\right)}}{2 \pi}=\lambda^{\left(L, U_{4}\right)} \mathbf{i d}_{\cdot 1 \times 1,4 \times 4},
$$

with some constants $\lambda^{(L)}$ and $\lambda^{\left(U_{4}\right)}$, which are equivalent to the Hermitian-Einstein condition for gauge fields

$$
\begin{aligned}
\frac{F^{(L)}}{2 \pi} \wedge J \wedge J & =\quad \lambda^{(L)} \text { id }_{\cdot 1 \times 1}\left(\frac{1}{3 !} J \wedge J \wedge J\right), \\
\frac{F^{\left(U_{4}\right)}}{2 \pi} \wedge J \wedge J & =-\frac{1}{4} \lambda^{(L)} \text { id. }_{4 \times 4}\left(\frac{1}{3 !} J \wedge J \wedge J\right) .
\end{aligned}
$$

\footnotetext{
${ }^{13}$ The D-term scalar potential after completing square in (58) does not depend on the choice of the overall normalisation of $\mathbf{q}_{\chi}$ and $q_{\chi, i}$. This is just a matter of how charges and coupling constant are separated in $\mathrm{U}(1)$ gauge theories.

${ }^{14} \xi=-\left(Q^{k} \partial K / \partial T^{k}+Q^{0} \partial K / \partial S\right)=-\left(Q^{k} \partial K / \partial T^{k \dagger}+Q^{0} \partial K / \partial S^{\dagger}\right)$ is also the same. The derivative of the Killing potential

$$
d \xi=-Q^{k}\left(K_{T^{k} T^{l \dagger}} d T^{l \dagger}+K_{T^{l} T^{k \dagger}} d T^{l}\right)-Q^{0}\left(K_{S S^{\dagger}} d S^{\dagger}+K_{S S^{\dagger}} d S\right)
$$

is consistent with the Killing vector $-i Q^{k}\left(\partial_{T^{k}}-\partial_{T^{k \dagger}}\right)-i Q^{0}\left(\partial_{S}-\partial_{S^{\dagger}}\right)$ corresponding to (52) and (51).
} 
The solutions to these equations minimize ${ }^{15}$ the D-term potential from the tree-level FayetIliopoulos parameter, leaving positive energy $\left(g_{\mathrm{YM}}^{2} / 80\right)\left|\xi_{\chi}\right|^{2}=(5 / 256) g_{\mathrm{YM}}^{2}\left(M_{G}^{2} l_{s}^{2} \lambda\right)^{2}$. If one finds a holomorphic stable vector bundle $U_{4}$, Donaldson-Uhlenbeck-Yau's theorem guarantees that there exists a Yang-Mills field configuration satisfying (61). There is no such subtlety for the holomorphic line bundle $L$ because it is stable by definition. Thus, a solution to the equation of motions exists for the reducible bundle (29), as long as $U_{4}$ is chosen to be a holomorphic stable bundle.

This solution, however, is not stable both in mathematical and physical sense. If $c_{1}(L) \wedge$ $J \wedge J=-c_{1}\left(U_{4}\right) \wedge J \wedge J \propto \lambda$ is not zero, the rank-5 reducible holomorphic bundle $V_{5}$ (29) is unstable in the mathematical definition. In physics language, this "instability" has a clear meaning. Non-zero $\lambda$ means a non-vanishing tree-level Fayet-Iliopoulos parameter (57), which requires (at least at the classical level, ignoring the 1-loop term in (57)) that either one of $\mathrm{U}(1)_{\chi}$-charged objects develop a non-zero expectation value to absorb the non-zero Dterm, further minimizing the entire D-term, $\xi_{\chi}$ and $q_{\chi, i} \psi_{i}^{\dagger} \psi_{i}$ combined. The field responsible for this can be either $\bar{N}$ or $\overline{\bar{N}}$, the off-diagonal blocks of the bundle group $\mathrm{SU}(5)$. The Fayet-Iliopoulos parameter triggers the $\mathrm{U}(1)_{\chi}$ symmetry breaking and the $\mathrm{U}(1)_{\chi}$-symmetric vacuum is not stable.

Another way to see the instability is to note that $F_{\alpha \bar{\beta}} g^{\bar{\beta} \alpha}$ in the $\mathrm{U}(1)_{\chi}$ direction gives rise to the difference between the equation of motions of vector fields and gauge fermions. Fermions and vector fields have the same property under the $\mathrm{SU}(3) \subset \mathrm{SO}(6)$ of the Lorentz group of the internal space, but not under the $\mathrm{U}(1) \subset \mathrm{U}(3) \subset \mathrm{SO}(6)$. When there is a zero mode of gauge fermion, its would-be supersymmetric partner has either positive or negative mass-squared in the equations of motions, depending on the $\mathrm{U}(1)_{\chi}$ charge $q_{\chi}$. This explains why either one of $\bar{N}$ or $\bar{N}$ have negative mass-squared when $\xi_{\chi}$ does not vanish. The cross terms of the D-term potential (58) lead to $m_{i}^{2} \propto \xi_{\chi} q_{\chi, i}$.

When $\int c_{1}(L) \wedge J \wedge J$ is negative, $\xi_{\chi}<0$, and one of the fields with positive $\mathrm{U}(1)_{\chi}$ charge, $\overline{\bar{N}}, \overline{\mathbf{5}}$ and $H(\mathbf{5})$, develops an expectation value. Supersymmetry-breaking masses of those

\footnotetext{
${ }^{15}$ Just like the equation of motion (59) is implemented as the minimization of the D-term potential in D $=4$ effective field theories, the condition for a bundle to be holomorphic (the absence of $(0,2)$ and $(2,0)$ components of the Yang-Mills field strength) is implemented as the F-term condition. Indeed, when the background Yang-Mills field configuration has a non-vanishing $(0,2)$ component, $W \ni \int \Omega \wedge(A\langle d A\rangle)$ gives rise to a term linear in $A$ in the superpotential. The F-term potential requires to minimize the coefficient of the linear term, the $(0,2)$ component of $\langle d A\rangle$. When the minimized $(0,2)$ component of a U(1) bundle does not vanish, i.e., when the "Fayet-Iliopoulos (or O'Raifeartaigh) F-term" does not vanish, a phase transition of the spontaneous $\mathrm{U}(1)$ symmetry breaking is triggered through the superpotential $W \ni \int \Omega \wedge(A\langle d A\rangle-A A A)$. The story here is completely in parallel with that of the D-term.
} 
fields determine which field gets the expectation value. ${ }^{16}$ Since the only possibility consistent with the real world is for $\bar{N}$ to develop an expectation value, we assume this in the following. This mathematically means that the rank-5 vector bundle $V_{5}$ is no longer a reducible bundle (29), but is given by an extension [19] of $U_{4}$ by $L$ :

$$
0 \rightarrow L \rightarrow V_{5} \rightarrow U_{4} \rightarrow 0
$$

If $\int c_{1}(L) \wedge J \wedge J>0$, then the rank-5 bundle is an extension of $L$ by $U_{4}$ :

$$
0 \rightarrow U_{4} \rightarrow V_{5} \rightarrow L \rightarrow 0
$$

We prefer for phenomenological reasons that right-handed neutrinos $\bar{N}$ do not acquire large expectation values. Since their expectation values provide large mass terms between $H_{u}$ and lepton doublets $L$, either $H_{u}$ or $L$ would be left out of the low-energy spectrum. Dimension-4 proton decay operators are also generated in this case, as we shall see later. Thus, $\int c_{1}(L) \wedge$ $J \wedge J$ should not be positive. ${ }^{17}$ If it is negative, then $h^{1}\left(Z, \bar{U}_{4} \otimes L\right)$ should be non-zero as well, so that $\bar{N}$ exists in the spectrum and absorbs the non-zero Fayet-Iliopoulos parameter. We shall see later that, in some cases, we can then relax the condition that the vector bundle be reducible without allowing a too rapid proton decay.

Before going directly to the discussion of proton decay operators controlled under the spontaneously broken anomalous $\mathrm{U}(1)_{\chi}$ symmetry, let us discuss the 1-loop term of the Fayet-Iliopoulos parameter (57). As one can see from [35], the 1-loop term in the Heterotic

\footnotetext{
${ }^{16}$ In the simplest situation where all these chiral multiplets have equal gravity-mediated supersymmetry breaking mass-squared, potential minimisation shows that only $\bar{N}$ develops a non-zero expectation value, none others. This is because $\overline{\bar{N}}$ has the largest positive the $\mathrm{U}(1)_{\chi}$ charge among them.

${ }^{17}$ Here, we implicitly assume that (44) and (46) are negative. If they are positive, the definition of the particles and anti-generation particles are exchanged, and now $\int_{Z} c_{1}(L) \wedge J \wedge J$ should not be negative.
} 
$E_{8} \times E_{8}$ theory $^{18}$ is proportional to the U(1)-SU $(5)_{\text {GUT }}^{2}$ mixed anomaly,

$$
\begin{aligned}
\sum_{i} q_{\chi, i} 2 T_{R, i} & =3 \times(\underline{34})-12 \times(\underline{35})-3 \times(\underline{36})+2 \times(\underline{37}) \\
& =\operatorname{tr}_{f}\left(\mathbf{q}_{\chi} \mathbf{q}_{L}\right) \int_{Z} c_{1}(L)\left(c_{2}\left(U_{4}\right)-c_{1}(L)^{2}-\frac{1}{2} c_{2}(T Z)\right) .
\end{aligned}
$$

Since we know that this anomaly vanishes for the $\mathrm{U}(1)_{\chi}$ symmetry in the spectrum of the minimal supersymmetric standard model (possibly with additional SU $(5)_{\text {GUT } \text {-singlets and }}$ vector-like pairs), the net 1-loop contribution is absent; $Q_{\chi}^{0}=0$. One can also see this explicitly by using (42) and (43) with $c_{2}\left(V_{5}\right)=c_{2}\left(U_{4}\right)-c_{1}(L)^{2}$. Therefore, no matter how moduli are stabilised (or even when moduli are not stabilised otherwise), the cancellation between the tree and 1-loop level contributions [35] cannot happen in realistic vacua satisfying (42) and (43).

Although we cannot expect cancellation between the two terms, the tree-level term itself may also vanish. The Kähler moduli can either be stabilised so that $\lambda$ in (60) vanishes or some of it remains unstable and the D-term scalar potential of the $U(1)_{\chi}$ symmetry may take the moduli into some region of the Kähler cone so that $\lambda$ in (60) vanishes. Either way, the Fayet-Iliopoulos parameter can vanish. In that case, some linear combination of the imaginary parts of the chiral multiplets $T^{k}$ 's is absorbed as the longitudinal mode [39, 38.

\footnotetext{
${ }^{18}$ In the compactification of Heterotic $\mathrm{SO}(32)$ theory with the spin connection embedded in the $\mathrm{SU}(3)$ subgroup of $\mathrm{SO}(6) \subset \mathrm{SO}(32)$, the bundle group leaves $\mathrm{SO}(26) \times \mathrm{U}(1)_{3}$ gauge symmetry unbroken. The $\mathrm{U}(1)_{3}$ factor is, however, anomalous; $Q_{3}^{k}=0$, but

$$
Q_{3}^{0}=\frac{c_{3}\left(V_{3}\right)}{8 \pi^{2}} .
$$

The corresponding Fayet-Iliopoulos parameter is given by $\xi=-M_{G}^{2} g_{\mathrm{YM}}^{2} \operatorname{tr}_{\mathrm{LE} .}\left(q_{3}\right) / 192 \pi^{2}$, after using a relation $\operatorname{tr}_{\mathrm{LE}}\left(q_{3}\right)=-(26-2) \chi\left(Z ; V_{3}\right)=-12 c_{3}\left(V_{3}\right)$; here, $\operatorname{tr}_{\mathrm{LE}} .\left(q_{3}\right)$ is the sum of $\mathrm{U}(1){ }_{3}$ charge in the low-energy spectrum. This Fayet-Iliopoulos parameter is proportional to the gravitational anomaly $\operatorname{tr}_{\mathrm{LE}}\left(q_{3}\right)$. In general, however, the 1-loop Fayet-Iliopoulos parameter is not expected to be proportional to gravitational anomaly; it is proportional only when the quadratic divergence of loops of all the charged particles are made finite exactly in the same way. In the $\mathrm{SU}(3)$-bundle compactification of the Heterotic $\mathrm{SO}(32)$ theory, it is the case: all the charged particles originate either from rank-3 bundle $V_{3}$ or from its Hermitian conjugate. In the case of our interest, however, various particles originate from totally different vector bundles, and we cannot expect that all the loops of low-energy particles are rendered finite in the same way. Perturbative Heterotic compactification can give rise to more than one anomalous U(1) gauge symmetries [35]; it is also the case in the model in our section [3.3. Their Fayet-Iliopoulos parameters can have both tree and 1-loop level contributions, either (or both) of them can vanish for certain choice of moduli parameters or matter contents, just like in Type I or Type II string theories.
} 
Since the kinetic term of the imaginary parts of $T^{k}$ 's are

$$
\begin{aligned}
\mathcal{L} & =-K_{k l}\left(\partial(\operatorname{Im} T)^{k}-Q^{k} A\right)\left(\partial(\operatorname{Im} T)^{l}-Q^{l} A\right) \\
K_{k l} & =M_{G}^{2}\left(2 \pi l_{s}^{2}\right)^{2} \frac{3}{2}\left[-\frac{\int \omega_{k} \wedge \omega_{l} \wedge J}{\int J^{3}}+\frac{3}{2} \frac{\int \omega_{k} J^{2} \int \omega_{l} J^{2}}{\left(\int J^{3}\right)^{2}}\right],
\end{aligned}
$$

and the mass-squared of the gauge field is roughly of the order

$$
m_{\chi}^{2} \approx g_{\mathrm{YM}}^{2} M_{G}^{2}\left(\frac{l_{s}}{R}\right)^{4},
$$

where $R$ is a "typical" radius of compactification manifold, assuming that it is isotropic. The real-part scalar corresponding to the absorbed combination acquires a mass-squared from the D-term scalar potential.

If all the Kähler moduli have already been stabilised by other means, such as nonperturbative potentials, then the tree-level Fayet-Iliopoulos parameter may not vanish. When $\xi_{\chi}$ is negative, a chiral multiplet $\overline{\bar{N}}$ of positive $\mathrm{U}(1)_{\chi}$ charge, +5 , develops an expectation value, absorbing negative $\xi_{\chi}$. In this case, the gauge boson absorbs some linear combination of $T^{k}$ 's and $\bar{N}$ and removes just one flat direction. The non-zero expectation value of $\mathrm{U}(1)_{\chi^{-}}$ charged particles, $\left\langle\overline{\bar{N}}^{\dagger} \overline{\bar{N}}\right\rangle \approx M_{G}^{2}\left(l_{s} / R\right)^{2} /(8 \pi)$, gives rise to another mass term of the $\mathrm{U}(1)_{\chi}$ gauge field:

$$
m_{\chi}^{2} \approx \frac{5 g_{\mathrm{YM}}^{2}}{32 \pi} M_{G}^{2}\left(\frac{l_{s}}{R}\right)^{2}=\frac{5 \pi}{2} \frac{1}{R^{2}} .
$$

For compactification manifold with $R$ sufficiently larger than $l_{s}$, the latter Higgs-mechanism contribution dominates over (68). The mass scale of the $\mathrm{U}(1)_{\chi}$ gauge boson is an important parameter in leptogenesis. From (69), we see that the mass is typically around the Kaluza-

Klein scale. Independent of whether $\xi=0$ or $\xi<0$, the anomalous $\mathrm{U}(1)_{\chi}$ gauge boson tends to have a very large mass, and easily escape the mass bound $m_{\chi} \gtrsim 600 \mathrm{GeV}$ [23] from low-energy experiments.

\subsubsection{Effective Superpotential}

Let us now turn our attention to the effective superpotential, with or without the expectation value of the scalar field in $\overline{\bar{N}}$. If both $\bar{N}$ or $\bar{N}$ have vanishing expectation value, the Dirac Yukawa couplings in (31) are the only sources of the masses of neutrinos. The U(1) $\chi$ symmetry remains unbroken as a global symmetry at the perturbative level, and forbids Majorana masses of right-handed and left-handed neutrinos. The dimension-4 proton decay 
operators are also forbidden. Although the $\mathrm{U}(1)_{\chi}$ symmetry is broken at non-perturbative level, the $\mathrm{SU}(4)$ structure group of the bundle $U_{4}$ still controls the effective operators. Any effective operators should be written by low-energy multiplets and spurions in the $\mathfrak{s u ( 4 ) - a d j}$. representation. The operators have to be symmetric under SU(4) and SU(5) $\mathrm{GUT}$ and SU(4) symmetry is broken only by the Yang-Mills field configuration, whose effects are taken into account by the spurions.

Under this constraint on the effective operators, one cannot find an operator for Majorana neutrino masses or dimension-4 proton decay operators. This is a blessing in a sense that proton does not decay too rapidly. On the other hand, the absence of the Majorana masses of neutrinos require ${ }^{19}$ that the Dirac Yukawa couplings of neutrinos be as small as $10^{-11}$ or even less, in order to account for the very tiny neutrino masses observed in neutrino oscillation experiments. The Dirac Yukawa couplings in (31) originate from $E_{8}$ Yang-Mills interactions. It is a challenging problem to find out how they can be so small.

Let us now consider a situation where either the anti-generation right-handed neutrinos $\overline{\bar{N}}$ or right-handed neutrinos $\bar{N}$ obtain non-zero expectation values. These situations correspond to $\int_{Z} c_{1}(L) \wedge J \wedge J<0$ and $\int_{Z} c_{1}(L) \wedge J \wedge J>0$, respectively.

Large expectation value in $\bar{N}$ are not welcomed phenomenologically, partly because we do not want large mass terms of the form $W \ni\langle\bar{N}\rangle \overline{\mathbf{5}} \cdot H(\mathbf{5})$. Not all the moduli of the rank-5 vector bundle $V_{5}$ turn on masses to all vector-like pair of $\mathrm{SU}(5)_{\mathrm{GUT}} \mathbf{- 5}+\overline{\mathbf{5}}$ representations 34 . Thus, there may be a way out of this problem.

Another serious problem is the dimension- 4 proton decay. In the rank- 5 vector bundle $V_{5}$ given as an extension of $L$ by $U_{4}$ as in (63), $U_{4}$ remains a sub-bundle, and $L$ ceases to be a sub-bundle of $V_{5}$. This means that the zero-mode wave functions of the $\mathrm{SU}(5)_{\mathrm{GUT}}-\mathbf{1 0}$ multiplets from the bundle $U_{4}$ can still be confined in $U_{4}$, but those of the $\mathrm{SU}(5)_{\mathrm{GUT}}-\mathbf{1 0}^{\prime}$ multiplets (if exist) are no longer confined in "a sub-bundle $L$." Rather they take values in the entire bundle $V_{5}$. The same is true for the wave functions of the $\mathrm{SU}(5)_{\mathrm{GUT}^{-}} \overline{\mathbf{5}}$ multiplets as they are no longer confined in "a sub-bundle $U_{4} \otimes L$ " of $\wedge^{2} V_{5}$, but take values in $U_{4} \otimes V_{5}$. In particular, the $\overline{\mathbf{5}}$ multiplets have wave functions partially in " $\wedge^{2} U_{4}$ " part and acquire properties of the $\bar{H}(\overline{\mathbf{5}})$ multiplet. Thus, the second term of (7) generates the dimension-4 proton decay operators by picking up the wave function of one of $\overline{\mathbf{5}}$ multiplets from the " $\wedge^{2} U_{4}$ " part, just like the down-type quark and charged-lepton Yukawa couplings are generated.

\footnotetext{
${ }^{19}$ The origin of baryon asymmetry of our universe is another problem in the pure Dirac scenario of the neutrino masses. Standard scenario of leptogenesis 40 does not work. Some ideas have been proposed for the leptogenesis in the scenario with Dirac neutrino masses [4].
} 
The effective-field theory language can also capture how the dimension-4 operators are generated. The expectation values in the vector bundle moduli $\bar{N}$ force the wave functions of zero-modes to be modified, and for $\overline{\mathbf{5}}$ multiplets, for instance, their new wave functions are something like

$$
\overline{\mathbf{5}}_{\text {new }} \approx \overline{\mathbf{5}}_{\text {red }}+\langle\bar{N}\rangle \overline{\mathbf{5}}_{\text {red }}
$$

treating the deviation from that in the reducible limit as a small perturbation. The second term takes its value in the " $\wedge^{2} U_{4}$ " part, like the $\bar{H}(\overline{\mathbf{5}})$ multiplet. Thus, effective operators

$$
W \ni \overline{\mathbf{5}}_{\text {red }} \cdot \mathbf{1 0} \text { red } \cdot\left(\langle\bar{N}\rangle \cdot \overline{\mathbf{5}}_{\text {red }}\right)
$$

that are invariant under the $\mathrm{SU}(4) \times \mathrm{U}(1)_{\chi}$ structure group at the reducible limit give rise to dimension-4 proton decay operators.

On the contrary, expectation values of $\bar{N}$ do not create this problem. The sub-bundle $L$ remains to be well-defined, and the wave functions of the $\overline{\mathbf{5}}$ multiplets are confined in $V_{5} \otimes L$, which does not contain a " $\wedge^{2} U_{4}$ " part that have properties of the $\bar{H}(\overline{\mathbf{5}})$ multiplet. The effective-field theory analysis using $\langle\bar{N}\rangle$ insertions leads to the same answer. ${ }^{20}$ In summary, in order to avoid dimension- 4 proton decay, a rank- 5 holomorphic stable vector bundle $V_{5}$ should have

a rank-1 sub-bundle $L \quad$ with $\left(\int_{Z} c_{1}(L) \wedge J \wedge J<0\right) \quad$ when $\int_{Z} c_{3}\left(V_{5}\right)<0$ a rank-4 sub-bundle $U_{4}$ with $\left(\int_{Z} c_{1}\left(\operatorname{det} U_{4}\right) \wedge J \wedge J<0\right) \quad$ when $\int_{Z} c_{3}\left(V_{5}\right)>0$.

It is interesting to see how the dimension- 4 operators are forbidden in the scenario with non-vanishing $\bar{N}$ expectation values. Two major ideas for getting rid of the dimension-4 operators have been the $R$-parity and $\mathbb{Z}_{2^{-}} \chi$ (or $B-L$ ) symmetry. But, the anti-generation right-handed neutrinos $\bar{N}$ have the same $R$-parity and $\mathbb{Z}_{2-} \chi$ charges as the right-handed neutrinos, and are odd. Thus, both symmetries are broken by the expectation value of $\bar{N}$, and the insertion of the expectation value can supply any $R$-parity or $\mathbb{Z}_{2^{-}} \chi$ charges in effective operators. It is rather the continuous $\mathrm{U}(1)_{\chi}$ symmetry that is broken only by a positively charged field that essentially protects protons from decaying rapidly. Since the symmetry breaking of $\mathrm{U}(1)_{\chi}$ (and $\left.B-L\right)$ is triggered not by an F-term, but by the D-term, fields with opposite charges do not have to develop expectation values even when the symmetry is spontaneously broken.

\footnotetext{
${ }^{20}$ As long as $\chi\left(\mathbf{1 0}^{\prime}\right)$ is negative, such chiral matters can form a mass term $W \ni \mathbf{1 0} \cdot\left(\langle\bar{N}\rangle \overline{\mathbf{1 0}^{\prime}}\right)$ and do not necessarily appear in the low-energy spectrum. Thus, (41) does not have to be imposed when the Fayet-Iliopoulos parameter is non-zero.
} 
Such terms in effective superpotential

$$
W \ni \kappa \overline{\bar{N}} \bar{N} \bar{N} \bar{N}
$$

are perfectly consistent with the underlying $E_{8}$ symmetry, and it is not surprising if such interactions are generated in some way (e.g. 42]). Once such terms are generated, the non-vanishing expectation value of $\overline{\bar{N}}$ gives rise to the Majorana mass term of right-handed neutrinos 43. Since the natural scale of the expectation value of $\overline{\bar{N}}$ is very high, the Majorana mass is also very large unless the coefficient of the operator (72) is extremely suppressed. Such heavy right-handed Majorana neutrinos naturally explain tiny left-handed neutrino masses observed in neutrino oscillation experiments through the see-saw mechanism [14]. A flat direction $\left\langle|\overline{\bar{N}}|^{2}\right\rangle-\left\langle|\bar{N}|^{2}\right\rangle=-\xi_{\chi} / 5$ is also removed by the operator (172), if it exists, and $\langle\bar{N}\rangle=0$ and $\left\langle|\overline{\bar{N}}|^{2}\right\rangle=-\xi_{\chi} / 5$ becomes the only solution. Therefore, if the operator (172) exists, it not only provides the Majorana masses of right-handed neutrinos and also explains tiny left-handed neutrino masses through the see-saw mechanism, but also prevents the dimension-4 proton decay operators from being generated.

We have seen that the Yang-Mills interaction $W \ni \int \Omega \wedge(A \wedge A \wedge A)$ does not generate dimension-4 proton decay operators when $\langle\bar{N}\rangle=0$ and $\langle\overline{\bar{N}}\rangle \neq 0$, or when the rank-5 bundle $V_{5}$ is given by the extension (62). The effective-field theory arguments are more powerful and say that no perturbative (tree level in practise) processes can give rise to the dangerous dimension-4 operators. Non-perturbative processes, however, may generate them. For example, an operator

$$
W \ni \epsilon \overline{\mathbf{5}} \cdot \mathbf{1 0} . \overline{\mathbf{5}} \cdot\langle\overline{\bar{N}}\rangle^{3}
$$

is invariant under $\mathrm{SU}(4)$ structure group and $\mathrm{SU}(5)_{\text {GUT }}$ symmetry, and is of $\mathrm{U}(1)_{\chi}$ charge 20. Since the chiral multiplets of Kähler moduli $T^{k}$ transform as in (52), if the coefficient $\epsilon$ contains a non-perturbative factor such as some linear combinations of $T^{k}$ 's on the exponent, $\mathrm{U}(1)_{\chi}$ charges may be supplied and the operator above is consistent with the underlying $\mathrm{SU}(4) \times \mathrm{U}(1)_{\chi} \times \mathrm{SU}(5)_{\text {GUT }} \subset E_{8}$ symmetry. World-sheet instanton 42 amplitudes have such properties, and in particular, an amplitude from a world-sheet wrapping on a curve $C$ has a prefactor

$$
\epsilon \propto \exp \left[\frac{1}{2 \pi \alpha^{\prime}} \int_{C}(-J+i B)\right]=\exp \left[(2 \pi)^{2} \int_{C} \omega_{k} T^{k}\right] .
$$

Such a prefactor transforms linearly under the $\mathrm{U}(1)_{\chi}$ gauge transformation with charge

$$
q_{\chi, C}=-(2 \pi)^{2} Q^{k} \int_{C} \omega_{k}=5 \int_{C} c_{1}(L) .
$$




\begin{tabular}{|c|c|c|c|c|c|c|}
\hline Bundles & $U_{2}$ & $U_{3}$ & $U_{3} \otimes U_{2}$ & $\overline{\wedge^{2} U_{2}}$ & $\wedge^{2} U_{3}$ & $U_{2} \otimes \overline{U_{3}}$ \\
\hline Particles ID A & $(U, Q, \bar{E})$ & $\left(\left(\mathbf{1 0}^{\prime}\right)\right)$ & $\mathbf{5}=(\bar{D}, L)$ & $H(\mathbf{5})$ & $\bar{H}(\mathbf{5})$ & $N$ \\
Particles ID B & $(\bar{U}, Q, \bar{E})$ & $\left(\left(\mathbf{1 0}^{\prime}\right)\right)$ & $\bar{H}(\overline{\mathbf{5}})$ & $H(\mathbf{5})$ & $\overline{\mathbf{5}}=(\bar{D}, L)$ & $S$ \\
\hline
\end{tabular}

Table 3: Particle identification in $\mathrm{SU}(3)_{2} \times \mathrm{SU}(2)_{2} \times \mathrm{U}(1)_{\tilde{q}_{7}}$ bundle compactification. There are 2 phenomenologically viable identifications. There are no particles in the $\mathrm{SU}(5)_{\mathrm{GUT}} \mathbf{- 1 \mathbf { 1 0 } ^ { \prime }}$ representation coming from the bundle $U_{3}$ in the low-energy spectrum of our world.

Thus, for instance, a dimension-7 operator like (73) may be generated from a curve $C$ with $\int_{C} c_{1}(L)=-4$. Such operators tend to be numerically suppressed because the prefactors are exponentially small $\exp \left[-\operatorname{vol}(C) /\left(2 \pi \alpha^{\prime}\right)\right]$ and it is not immediately clear whether such operators are excluded by the experimental limits by the data available so far.

\subsection{Compactification with $3+2$ Vector Bundles}

\subsubsection{Spectrum}

If the reducible rank-5 vector bundle (30) with the structure group $\mathrm{SU}(3)_{2} \times \mathrm{SU}(2)_{2} \times \mathrm{U}(1)_{\tilde{q}_{7}}$ is introduced instead of $\mathrm{SU}(4) \times \mathrm{U}(1)_{\chi}$ bundle (29), the origin of the low-energy particles are identified with the irreducible bundles shown in Table 3. Discussion in section 2 guarantees that the trilinear Yukawa couplings of quarks and leptons arise from the $E_{8}$ Yang-Mills interactions, while the dimension- 4 operators for proton decay remain absent.

Chirality formulae are given by

$$
\begin{aligned}
\chi\left(U_{2}\right) & =-\frac{1}{12} c_{2}(T Z) c_{1}\left(U_{3}\right)-\frac{1}{6} c_{1}\left(U_{3}\right)^{3}+\frac{1}{2} c_{2}\left(U_{2}\right) c_{1}\left(U_{3}\right), \\
\chi\left(U_{3}\right) & =+\frac{1}{12} c_{2}(T Z) c_{1}\left(U_{3}\right)+\frac{1}{6} c_{1}\left(U_{3}\right)^{3}-\frac{1}{2} c_{2}\left(U_{3}\right) c_{1}\left(U_{3}\right)+\frac{1}{2} c_{3}\left(U_{3}\right), \\
\chi\left(\overline{\wedge^{2} U_{2}}\right) & =+\frac{1}{12} c_{2}(T Z) c_{1}\left(U_{3}\right)+\frac{1}{6} c_{1}\left(U_{3}\right)^{3}, \\
\chi\left(U_{3} \otimes U_{2}\right) & =-\frac{1}{12} c_{2}(T Z) c_{1}\left(U_{3}\right)-\frac{1}{6} c_{1}\left(U_{3}\right)^{3}+\frac{1}{2} c_{2}\left(U_{2}\right) c_{1}\left(U_{3}\right)+c_{3}\left(U_{3}\right), \\
\chi\left(\wedge^{2} U_{3}\right) & =+\frac{2}{12} c_{2}(T Z) c_{1}\left(U_{3}\right)+\frac{2}{6} c_{1}\left(U_{3}\right)^{3}-\frac{1}{2} c_{2}\left(U_{3}\right) c_{1}\left(U_{3}\right)-\frac{1}{2} c_{3}\left(U_{3}\right), \\
\chi\left(U_{2} \otimes \bar{U}_{3}\right) & =-\frac{5}{12} c_{2}(T Z) c_{1}\left(U_{3}\right)-\frac{11}{6} c_{1}\left(U_{3}\right)^{3}+\frac{5}{2} c_{2}\left(U_{3}\right) c_{1}\left(U_{3}\right)-c_{3}\left(U_{3}\right) \\
& +\frac{4}{2} c_{2}\left(U_{2}\right) c_{1}\left(U_{3}\right) .
\end{aligned}
$$

Here, $c_{1}\left(U_{2}\right)=-c_{1}\left(U_{3}\right)$ is used. 
The same argument leading to (411) requires

$$
\chi\left(U_{3}\right)=0,
$$

and the same net chirality for $\mathbf{1 0}$ and $\overline{\mathbf{5}}$ (or equivalently for $H(\mathbf{5})$ and $\bar{H}(\overline{\mathbf{5}})$ ) requires

$$
\chi\left(U_{2}\right)=\chi\left(U_{3} \otimes U_{2}\right), \quad \text { or equivalently } \quad \chi\left(\operatorname{det} U_{2}^{-1}\right)=\chi\left(\wedge^{2} U_{3}\right)
$$

under the particle identification A in Table 3 . These phenomenological information constrains the possible choice of topology for our vacuum as

$$
\begin{aligned}
c_{2}(T Z) c_{1}\left(U_{3}\right) & =\left[6 c_{2}\left(U_{3}\right)-2 c_{1}\left(U_{3}\right)^{2}\right] c_{1}\left(U_{3}\right), \\
c_{3}\left(U_{3}\right) & =0,
\end{aligned}
$$

where we used $c_{1}\left(U_{2}\right)=-c_{1}\left(U_{3}\right)$. Under these conditions we have

$$
\begin{aligned}
\chi(\mathbf{1 0})=\chi(\overline{\mathbf{5}}) & =\frac{1}{2}\left[c_{2}\left(U_{2}\right)-c_{2}\left(U_{3}\right)\right] c_{1}\left(U_{3}\right), \\
\chi(H(\mathbf{5}))=\chi(\bar{H}(\overline{\mathbf{5}})) & =\frac{1}{2} c_{2}\left(U_{3}\right) c_{1}\left(U_{3}\right), \\
\chi(\bar{N}) & =-c_{1}\left(U_{3}\right)^{3}-\frac{1}{2} c_{2}\left(U_{3}\right) c_{1}\left(U_{3}\right)+\frac{5}{2} c_{2}\left(U_{2}\right) c_{1}\left(U_{3}\right) .
\end{aligned}
$$

Similar analysis can be done for the case of the identification B in Table 3

\subsubsection{Anomalous $\mathrm{U}(1)_{\tilde{q}_{7}}$ Symmetry and Effective Superpotential}

The $\mathrm{U}(1)_{\tilde{q}_{7}}$ gauge field has Green-Schwarz coupling because of non-trivial $c_{1}\left(U_{3}\right)=-c_{1}\left(U_{2}\right)$, just like the gauge field of the $\mathrm{U}(1)_{\chi}$ symmetry does in section 3.1.2 Essentially the same analysis can be done for the $\mathrm{U}(1)_{\tilde{q}_{7}}$ symmetry. The gauge boson acquires a large mass either from the Green-Schwarz interactions or from the Higgs mechanism, and can be absent from low-energy spectrum. In this section, we just only refer to the difference from what was discussed in 3.1.2

The Fayet-Iliopoulos parameter given in (57) is valid for the $\mathrm{U}(1)_{\tilde{q}_{7}}$ symmetry after replacing $c_{1}(L)$ by $c_{1}\left(U_{3}\right)$; the prefactor 10 happens to be the same. The 1-loop contribution does not necessarily vanish for the $\mathrm{U}(1)_{\tilde{q}_{7}}$ symmetry, and the Fayet-Iliopoulos parameter vanishes when the tree-level and 1-loop contributions cancel [35]. In that case, the reducible vector bundle (30) is semi-stable at the 1-loop level. 
When the bundle (30) is at the reducible limit, not only the dimension-4 but also dimension5 proton decay operators are forbidden by the underlying symmetry. Indeed, neither

$$
U_{2} \otimes U_{2} \otimes U_{2} \otimes\left(U_{3} \otimes U_{2}\right) \quad[\mathrm{ID} A], \quad \text { nor } \quad \mathrm{U}_{2} \otimes \mathrm{U}_{2} \otimes \mathrm{U}_{2} \otimes\left(\wedge^{2} \mathrm{U}_{3}\right) \quad[\mathrm{ID} \mathrm{B}]
$$

contains a trivial bundle. Since these bundles are not even singlet under $\mathrm{SU}(3)_{2}$, these operators are forbidden even at the non-perturbative level.

The particle identification B in Table 3 may yield chiral multiplets $S$. Since we have not made any specific choices of vector bundles, we do not know how many chiral multiplets are available (or if there is a vacuum without such a multiplet) around the electroweak scale. But, if there are, then they play some roles in the Higgs-related physics, since they has a coupling $W \ni S H_{u} H_{d}$. Thus, it is interesting to know more about the interactions of the multiplet $S$. If there are only multiplets $S$, without their Hermitian-conjugate $\bar{S}$, then the underlying $\mathrm{SU}(3)_{2} \times \mathrm{SU}(2)_{2} \times \mathrm{U}(1)_{\tilde{q}_{7}}$ symmetry kills any kinds of terms in the superpotential only in $S$. At the non-perturbative level, where we ignore the $\mathrm{U}(1)_{\tilde{q}_{7}}$-charge conservation, some terms may be generated:

$$
W \ni S H_{u} H_{d}+\epsilon S^{6}+\epsilon^{\prime} S^{12}+\cdots
$$

The dimension-7 term $W \ni S^{6}$ is the leading correction, and the cubic term $W \ni S^{3}$ of the next-to-minimal supersymmetric standard model [27] is not allowed even at the nonperturbative level. ${ }^{21}$ Phenomenology of an effective theory without $S^{3}$ term has been studied in [28, 29].

If the values of Kähler moduli and dilaton are such that the Fayet-Iliopoulos parameter does not vanish, then the $\mathrm{U}(1)_{\tilde{q}_{7}}$ symmetry is spontaneously broken. The absence of dimension-4 proton decay operators suggests the presence of the Fayet-Iliopoulos parameter. Under the identification A in Table $3,\langle\overline{\bar{N}}\rangle=0,\langle\bar{N}\rangle \neq 0$ and the rank-5 bundle is an extension

$$
0 \rightarrow U_{2} \rightarrow V_{5} \rightarrow U_{3} \rightarrow 0
$$

The bundle $U_{2}$ has to remain well-defined as a sub-bundle, so that the $\overline{\mathbf{5}}$-representation is still distinguished from $\bar{H}(\overline{\mathbf{5}})$. Under the identification $\mathrm{B},\langle S\rangle=0,\langle\bar{S}\rangle \neq 0$ and the rank-5 bundle is an extension

$$
0 \rightarrow U_{3} \rightarrow V_{5} \rightarrow U_{2} \rightarrow 0
$$

\footnotetext{
${ }^{21}$ Here, we assume that the underlying $E_{8}$ symmetry is broken only by the vector bundle, and not directly by any non-perturbative processes.
} 
The bundle $U_{3}$ has to be a well-defined sub-bundle for the same reason as in the case of the identification A.

If the Fayet-Iliopoulos parameter is of order $M_{G}^{2}\left(l_{s} / R\right)^{2}$, as indicated from the tree-level contribution, it is not small as long as $1 / R$ is around the GUT scale. Thus, the expectation values of $\bar{N}$ and $\bar{S}$ are much larger than the electroweak scale. In the former case, $\overline{\mathbf{5}}$ and $H(5)$ multiplets decouple from the low-energy physics in pair, by acquiring mass terms from the Dirac Yukawa coupling with the expectation value of $\langle N\rangle$. The expectation value of a vector bundle moduli $\bar{N}$, however, does not necessarily add masses to all the vector-like pair in the $\mathrm{SU}(5)_{\mathrm{GUT}^{-}} \mathbf{5}+\overline{\mathbf{5}}$ representations [34]. Thus, some of those multiplets may remain in the low-energy spectrum, and a large expectation value in one of $\bar{N}$ may not be inconsistent with the reality. The analysis based on (83) does not take account of this, and the constraints on topology obtained there have to be modified appropriately.

Neutrinos may have masses through

$$
W \ni \frac{1}{2}\left(L, \bar{N}^{\prime}, \overline{\bar{N}}\right)\left(\begin{array}{ccc}
0 & y\left\langle H_{u}\right\rangle & 0 \\
y\left\langle H_{u}\right\rangle & 0 & M \\
0 & M & \kappa\left\langle\bar{N}^{2}\right\rangle
\end{array}\right)\left(\begin{array}{c}
L \\
\bar{N}^{\prime} \\
\overline{\bar{N}}
\end{array}\right)
$$

under the identification A, where a vector-like mass term $W \ni M \bar{N}^{\prime} \overline{\bar{N}}$ and an operator like (72) exist. $\bar{N}^{\prime}$ collectively stand for chiral multiplets from the bundle $U_{2} \otimes \overline{U_{3}}$ except the one that develops an expectation value to absorb the Fayet-Iliopoulos parameter. This type of mass matrix explains the tiny neutrino masses through so-called the double-see-saw mechanism. On the other hand, in the identification B, the origin of neutrino masses is not understood.

Effective field theory analysis can be carried out for the dimension-5 proton decay operators by allowing the expectation values of $\bar{N}$ or $\bar{S}$ to be inserted in effective operators. It turns out that the underlying symmetry $\mathrm{SU}(5)_{\mathrm{GUT}} \times \mathrm{SU}(3)_{2} \times \mathrm{SU}(2)_{2} \times \mathrm{U}(1)_{\tilde{q}_{7}}$ in the identification A does not allow such an operator. Hence the dimension-5 proton decay is absent at the perturbative level. On the other hand, in the identification B, effective operators of the form

$$
W \ni \text { 10.10. }(10\langle\bar{S}\rangle) . \overline{5}
$$

are consistent with the symmetry $\mathrm{SU}(5)_{\mathrm{GUT}} \times \mathrm{SU}(3)_{2} \times \mathrm{SU}(2)_{2} \times \mathrm{U}(1)_{\tilde{q}_{7}}$, and hence may be generated at the perturbative level. Since the experimental limit on the dimension- 5 proton decay is not very severe, such operators may be consistent with the reality if the FayetIliopoulos parameter is not very large. 


\subsection{Compactification with $2+2+1$ Vector Bundles}

In $2+2+1$ bundle compactifications, $H(\mathbf{5})$ and $\bar{H}(\overline{\mathbf{5}})$ do not arise from a Hermitian-conjugate pair of irreducible representations, just like in $3+2$ bundle compactifications. Thus, the Wilson line cannot work as a solution to the doublet-triplet splitting problem. Not only dimension-4 proton decay operators but also dimension-5 operators are forbidden by the underlying symmetry, just like in the $3+2$ bundle compactifications. Since the $2+2+1$ vector bundles are regarded as particular choice of vector-bundle moduli of $3+2$ bundles and $4+1$ bundles, they inherit stronger properties of $3+2$ and $4+1$ bundles.

When the vector-bundle moduli are at the reducible limit, there are two linearly independent anomalous $\mathrm{U}(1)$ symmetries: $q_{6}$ and $q_{7}, \chi$ and $\psi$, or $\tilde{q}_{6}$ and $\tilde{q}_{7}$. When the Fayet-Iliopoulos parameters of those U(1) symmetries are non-zero, some of fields in the off-diagonal blocks in (21) develop non-zero expectation values so that the D-term potential energy vanishes. Depending on which field does, different types of effective operators are potentially generated. Which field develops an expectation value depends also on supersymmetry breaking masses, and we are not going into that discussion here.

\section{M-theory Vacua}

In this section, we will provide M-theory implementations of the general idea for the origin of low-energy particles and Yukawa couplings developed in terms of field theory in section 2. The $E_{7}$ or $E_{8}$ vector multiplet does not exist in the eleven-dimensional supergravity, as opposed to the case in Heterotic theory where $E_{8}$ vector multiplet exists from the beginning. In $M$ theory vacua, the $E_{7}$ or $E_{8}$ symmetry has to arise from singularity of $G_{2}$ holonomy manifold $^{22}$ used for the compactification yielding a vacuum with $\mathcal{N}=1$ supersymmetry. The geometry of the compactification manifold has to be properly engineered to obtain the right particle spectrum and the right form of interactions at low energies. In this section we present the geometric origin of the low-energy particles and of the absence of the very rapid proton decay.

It is known that the compactification of Heterotic String on $T^{3}$ is dual to M-theory compactification on $K 3$ [15]. This duality is further exploited by fibring $T^{3}$ and $K 3$ on a common base manifold $Q$. When $Q$ is a 3 dimensional, Heterotic compactification on $T^{3}$ fibred Calabi-Yau 3-fold and M-theory compactification on $K 3$-fibred $G_{2}$ holonomy manifold

\footnotetext{
${ }^{22}$ We abuse the word "manifold" even when a "manifold" has singularity.
} 
become relevant to the description of the real world (if low-energy supersymmetry is confirmed in a near future experiment). Neither all the Calabi-Yau 3-fold have $T^{3}$-fibred structure, nor all the $G_{2}$ holonomy manifold have $K 3$-fibred structure. Thus, some vacua have descriptions both in terms of Heterotic theory and M-theory, some may have only one in Heterotic theory, others may be described only in terms of M-theory compactification. Such vacua in different parts of the moduli space of the entire string theory may have different phenomenological aspects, especially when it comes to the pattern of Yukawa matrices and this is why we consider that it is important not only to develop string phenomenology in Heterotic theory but also in M-theory compactification in order to cover more vacua and more variety in phenomenology.

\subsection{Local Model of Up-type Quark Yukawa Coupling}

By comparing with complex geometries as Calabi-Yau 3-folds or 4-folds, little is known about the classification of $G_{2}$ holonomy manifold. Thus, it does not seem promising to search for realistic M-theory vacua in a top down approach. Although M-theory vacua with Heterotic dual may be studied by using the terminology of complex geometry in the Heterotic side, this approach does not suit for the purpose of exploring M-theory vacua that may not have Heterotic dual descriptions. What is more, we do not necessarily have to start in M-theory with an $E_{8}$ symmetry, as we saw in section 2 where $E_{7}$ was the minimal choice for the visible particle physics sector. Inflation and the supersymmetry breaking sector does not have to be constructed out of $E_{8}$ symmetric theory.

We adopt the philosophy of a local construction approach (or bottom-up construction approach) [44, instead. In M-theory and F-theory vacua, the $\mathrm{SU}(5)_{\text {GUT }}$ gauge fields propagate on a subvariety of the compactification geometry and the geometry (and D-brane configuration in Type IIA and Type IIB string theories) that is not in direct contact with the cycles of the visible sector has little effect on the visible sector. Since virtually no constraint comes from observational or experimental tests for the invisible sectors, the geometry of that part is almost arbitrary. In order to study various aspects of particle physics, it is only sufficient to construct the part of geometries that relates to experiments. One can neglect at this stage the mechanism of supersymmetry breaking and concentrate on the particle physics of quarks and leptons. By doing so, one can capture some generic features of phenomenol-

ogy, while avoiding the limitation to a small subclass of geometries and of phenomenology 
models. ${ }^{23}$ This is the basic philosophy of the local construction. ${ }^{24}$ One can start with a configuration with an appropriate gauge symmetry, and add the matter content one by one. Matters are added not by hand, but by constructing geometry, and the anomaly cancellation condition is replaced by consistency conditions of geometry. They are the difference from conventional model building in effective field theories. References [46] are notable examples of this approach in the context of $\mathrm{M} / G_{2}$ compactification.

The subject of relating compactifications of M-theory and particle physics has been treated extensively in the last years, see [50] for a review. The geometric building blocks are used in this section to implement our ideas of section 2 in M-theory compactification. The goal is

i) to choose the right underlying symmetry and its breaking pattern,

ii) to maintain different origin for $\overline{\mathbf{5}}$ and $\bar{H}(\overline{\mathbf{5}})$ in order to avoid a too fast dimension-4 proton decay,

iii) to obtain all the necessary Yukawa couplings generated from Yang-Mills interactions.

Let us first construct the local model of the up-type quark Yukawa coupling in M-theory, as we did in section 2. In M-theory description, the $E_{6}$ symmetry is realised by compactifying the M-theory on an ALE space containing six 2-cycles with the $E_{6}$-type intersection form. M2-branes wrapped on the 2-cycles correspond to gauge fields in the simple roots assigned to the nodes of the Dynkin diagram (see Fig. 1). The $\mathrm{D}=4$ effective theories with $\mathcal{N}=1$ supersymmetry are obtained by compactifying the M-theory on a $G_{2}$ holonomy manifold $X$. We are interested in a manifold $X$ with an associative 3-cycle $Q$ such that $X$ is locally ALE fibration over $Q$ with the six 2-cycles in the fibre. Four of them- $C_{3}, C_{4}, C_{5}$ and $C_{6}$-are not resolved anywhere on $Q$. The locus of the $A_{4}$ singularity is identified with $Q$ itself. The $\mathrm{SU}(5)_{\text {GUT }}$ vector multiplet of $\mathcal{N}=1$ supersymmetry is localised along this locus [51].

In order to describe the geometry for matter fields explicitly, we use the following description of ALE spaces [52] (for a brief summary, see appendix B). $E_{6}$ type ALE space and its metric are encoded by data $\vec{\zeta}^{i}=\left(\zeta_{1}^{i}, \zeta_{2}^{i}, \zeta_{3}^{i}\right) \in \mathbb{R}^{3}$ for $i=0, \cdots, 6$, which are under a constraint

$$
\vec{\zeta}^{0}+\vec{\zeta}^{1}+2 \vec{\zeta}^{2}+3 \vec{\zeta}^{3}+2 \vec{\zeta}^{4}+\vec{\zeta}^{5}+2 \vec{\zeta}^{6}=0 .
$$

Each of $\vec{\zeta}^{i}$ describes the "size" of the corresponding 2-cycle $C_{i}$, and this is also true for $i=0$, if we consider that $\alpha_{0} \equiv-\theta$ and $C_{0} \equiv C_{-\theta} \equiv-\left(C_{1}+2 C_{2}+3 C_{3}+2 C_{4}+C_{5}+2 C_{6}\right)$, where $-\theta$ is the negative of the highest root. The ALE space has an $A_{4}$ singularity when

\footnotetext{
${ }^{23}$ Needless to say, constructing explicit geometry is also important, to confirm that one has concrete examples in order to gain a firm understanding of what is really going on.

${ }^{24}$ Other approach to using local geometries to get phenomenology models appeared in [45].
} 


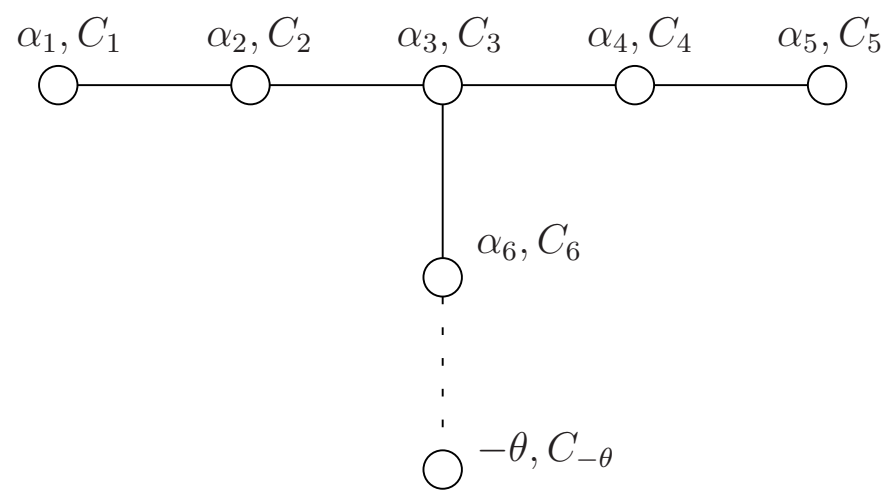

Figure 1: The (extended) Dynkin diagram of $E_{6}$, which not only describes the Lie algebra of $E_{6}$ symmetry but also the intersection form of $E_{6}$-type singularity. Each node is assigned a simple root $\alpha_{i}$ and a 2-cycle $C_{i}$. The $\mathfrak{s u}(2)_{2}$ and $\mathfrak{s u}(5)_{\text {GUT }}$ subalgebra of $\mathfrak{s u}(2)_{2}+\mathfrak{u}(1)+$ $\mathfrak{s u}(5)_{\text {GUT }} \subset \mathfrak{e}_{6}$ in section 2 are generated by $\alpha_{1}$ and $\alpha_{3,4,5,6}$, respectively. The highest root $\theta$ is not linearly independent from other six simple roots: $\theta=\alpha_{1}+2 \alpha_{2}+3 \alpha_{3}+2 \alpha_{4}+\alpha_{5}+2 \alpha_{6}$.

$\vec{\zeta}^{3}=\vec{\zeta}^{4}=\vec{\zeta}^{5}=\vec{\zeta}^{6}=\overrightarrow{0}$, and the 2-cycles $C_{3}, C_{4}, C_{5}$ and $C_{6}$ are of zero size.

Let $\vec{y}=\left(y_{1}, y_{2}, y_{3}\right)$ be a set of local coordinates of the associative 3 -cycle $Q$. The ALE fibre is allowed to vary over the base manifold $Q$, while keeping the $E_{6}$ intersection form and $A_{4}$ singularity for the SU(5) GUT gauge field. Thus, the local geometry of $G_{2}$ holonomy of our interest is described by specifying $\vec{\zeta}^{1}(\vec{y})$ and $\vec{\zeta}^{2}(\vec{y})$ as functions on $Q \cdot \vec{\zeta}^{0}(\vec{y})$ is determined by (95). The base manifold $Q$ is identified with the locus of $A_{4}$ singularity.

At some points on the base 3-fold $Q, \vec{\zeta}^{2}(\vec{y})$ or $\vec{\zeta}^{1}(\vec{y})+\vec{\zeta}^{2}(\vec{y})$ may become $\overrightarrow{0}$. There, either $C_{2}$ or $C_{1}+C_{2}$ shrinks. Since the intersection form of $C_{3}, C_{4}, C_{5}$ and $C_{6}$ along with an extra vanishing cycle $C_{2}$ or $C_{1}+C_{2}$ is the negative of the $D_{5}$ Cartan matrix, the $A_{4}$ singularity on $Q$ is enhanced to $D_{5}$ at such points. The local geometry around the point of enhanced gauge symmetry has a Type IIA interpretation, after reduction along the $S^{1}$ fibre; five D6-branes are on top of one another, and intersect with an O6-plane 81. One massless chiral multiplet either in the $\mathrm{SU}(5)_{\mathrm{GUT}}-\overline{\mathbf{1 0}}$ representation or in the $\mathrm{SU}(5)_{\mathrm{GUT}^{-}} \mathbf{1 0}$ is localised at the locus of enhanced gauge symmetry (singularity) [53. The chirality depends on the sign of Jacobian $\left|\partial \vec{\zeta}^{2}(\vec{y}) / \partial \vec{y}\right|$ or $\left|\partial\left(\vec{\zeta}^{1}(\vec{y})+\vec{\zeta}^{2}(\vec{y})\right) / \partial \vec{y}\right|$ at the loci of enhanced singularity.

The local description of the ALE fibration with local coordinates $\left(y_{1}, y_{2}, y_{3}\right)$ and data $\vec{\zeta}^{1}(\vec{y})$ and $\vec{\zeta}^{2}(\vec{y})$ is glued together between adjacent patches on $Q$ to give the global description of the ALE fibration over the entire $Q$. When two adjacent descriptions are glued, the 2-cycles on one patch should be matched with those of the other so that the intersection form is preserved, and further more, $C_{3}, C_{4}, C_{5}$ and $C_{6}$ should be matched with those of the other, 
since they have zero size. Furthermore, over the entire $Q$ we need to make sure that $C_{5}$ and $C_{6}$ remain the same everywhere on $Q$. In principle, one can trace $C_{6}$ moving over $Q$ and when one comes back to the original point on $Q, C_{6}$ may have become $C_{5}$. The $A_{4}$ intersection form is preserved through the entire process. But, in such geometry, not all of the SU(5) GUT gauge symmetry is maintained, as in the non-split case discussed in [26]. This is not what we want for the description of our world.

For the cycles $C_{2}$ and $C_{1}$, there is a little more degree of freedom in how to glue them between two adjacent patches. The Weyl reflection associated with the root $\alpha_{1}$ sends $\alpha_{2}$ to $\alpha_{1}+\alpha_{2}$, and $\alpha_{1}$ to $-\alpha_{1}$, keeping all other simple roots intact. This corresponds to a different choice of a Weyl chamber, and we could have chosen $-\alpha_{1}$ instead of $\alpha_{1}$, and $\alpha_{1}+\alpha_{2}$ instead of $\alpha_{2}$ as the simple roots. The same is true for the choice of independent homology basis of the ALE space. The intersection form is preserved under the "Weyl reflection" among the 2-cycles. The data may be matched on a common subset of a patch $\alpha$ and $\beta$ as

$$
\left(\begin{array}{c}
\vec{\zeta}^{2}(\vec{y}) \\
\vec{\zeta}^{1}(\vec{y})+\vec{\zeta}^{2}(\vec{y})
\end{array}\right)_{\alpha}=\left(\begin{array}{ll}
1 & \\
& 1
\end{array}\right)\left(\begin{array}{c}
\vec{\zeta}^{2}(\vec{y}) \\
\vec{\zeta}^{1}(\vec{y})+\vec{\zeta}^{2}(\vec{y})
\end{array}\right)_{\beta}
$$

or

$$
\left(\begin{array}{c}
\vec{\zeta}^{2}(\vec{y}) \\
\vec{\zeta}^{1}(\vec{y})+\vec{\zeta}^{2}(\vec{y})
\end{array}\right)_{\alpha}=\left(\begin{array}{c}
1 \\
1
\end{array}\right)\left(\begin{array}{c}
\vec{\zeta}^{2}(\vec{y}) \\
\vec{\zeta}^{1}(\vec{y})+\vec{\zeta}^{2}(\vec{y})
\end{array}\right)_{\beta}
$$

Thus, the 2-cycle $C_{2}$ on one patch may be $C_{1}+C_{2}$ on another, and vice versa. Thus, in the case the distinction between $C_{2}$ and $C_{1}+C_{2}$ is lost globally on $Q$ and there is no distinction at all between the $\mathrm{SU}(5)_{\mathrm{GUT}} \mathbf{- 1 0}$ representations arising from $C_{2}$-collapsed singularities and those from $\left(C_{1}+C_{2}\right)$-collapsed singularities. The doublet of data $\left(\vec{\zeta}^{2}(\vec{y}), \vec{\zeta}^{1}(\vec{y})+\vec{\zeta}^{2}(\vec{y})\right)$ becomes a 2-fold cover over $Q$, on which $\mathfrak{S}_{2}^{(2)}$, the Weyl group of $\mathrm{SU}(2)_{2}$ acts. The fact that the 10 representations arise from either $C_{2}$ or $\left(C_{1}+C_{2}\right)$-collapsed singularities reflects that these representation comes from the $(\mathbf{1 0}, \mathbf{2})$ irreducible component of $\mathfrak{e}_{6}$-adj. under the $\mathrm{SU}(2)_{2} \times \mathrm{U}(1)_{6} \times \mathrm{SU}(5)_{\text {GUT }}$ subgroup. The 2-fold cover describes the degree of freedom of the geometry for the M-theory compactification, just like did the spectral cover for the description of vector bundles on elliptic fibred manifolds in Heterotic theory. It is not surprising since the Heterotic-M-theory duality holds between Heterotic compactifications on $T^{3}$-fibred CalabiYau 3-folds and M-theory compactifications on $K 3$-fibred $G_{2}$ holonomy manifolds. But the description in terms of the 2 -fold cover is general in any M-theory compactifications on $G_{2}$ holonomy manifolds, not only for those with the $K 3$-fibration structure.

Now let us move on to another matter multiplet, $H(5)$. The 2 -cycle $C_{-\theta}$ shrinks at points where $\vec{\zeta}^{0}(\vec{y})=-\left(\overrightarrow{\zeta^{1}}(\vec{y})+2 \vec{\zeta}^{2}(\vec{y})\right)$ becomes $\overrightarrow{0}$. We can also say that the 2-cycle $\left(C_{1}+2 C_{2}\right)$ 
shrinks there. The intersection form of the four 2-cycles $C_{3}, C_{4}, C_{5}$ and $C_{6}$, along with another vanishing 2-cycle $C_{-\theta}$ is the negative of $A_{5}$ Cartan matrix. The singularity is enhanced from $A_{4}$ to $A_{5}$. The Type IIA interpretation locally exists: one D6-brane intersects at this point with a stack of five D6-branes, and one massless chiral multiplet either in the SU(5) GUT $^{-}$ $\mathbf{5}$ or $-\overline{\mathbf{5}}$ representation is localised there. The chirality depends on the sign of Jacobian $\left|\partial\left(\vec{\zeta}^{1}(\vec{y})+2 \vec{\zeta}^{2}(\vec{y})\right) / \partial \vec{y}\right|$ at the intersection point and, if the chirality comes out right, we have a candidate for $H(5)$. The irreducible decomposition of the $\mathfrak{e}_{6}$-adj. algebra along the the particle identification in section 2 also says that the $H(\mathbf{5})$ multiplet should come from the irreducible piece with roots $\alpha_{1}+2 \alpha_{2}$ in addition to some linear combinations of $\alpha_{3, \cdots, 7}$, confirming that this is the right geometric origin of $H(\mathbf{5})$ in the M-theory description.

Since the existence of massless chiral multiplets and their chirality depend only on the local geometry, the D6-brane and O6-plane interpretation that exists only locally can determine the multiplicity and chirality of massless multiplets, despite the absence of global Type IIA interpretation in compactification involving $E_{d^{-}}$type intersection form.

We have seen that there are candidates for $\mathbf{1 0}$ and $H(5)$ multiplets in the M-theory compactified on an $E_{6}$-type ALE fibration on a 3-cycle. The up-type quark Yukawa coupling (8) exists because the sum of 2-cycles $-C_{2},-\left(C_{1}+C_{2}\right)$ and $\left(C_{1}+2 C_{2}\right)$ is topologically trivial; M2-branes wrapped on these 2-cycles can merge together to disappear. Thus, the candidates really have the right properties to be $\mathbf{1 0}$ 's and $H(5)$.

When the $S^{1}$ fibre of the ALE space is negligibly small, this is qualitatively similar to the Type IIA description of the origin of trilinear Yukawa couplings: a worldsheet spanning 3 points of D6-D6 intersections. The Yukawa coupling is exponentially small, if the area spanned by the worldsheet is large. When the $S^{1}$ fibre is not necessarily small, the area is replaced by 3 -volume swept by a M2-brane. The Yukawa couplings have non-zero complex phases when the integration of the Ramond-Ramond 3-form over the 3-volumes $\int C^{(3)}$ are non-zero. Since the relevant 3-volumes are different for different entries of up-type quark Yukawa matrix, different entries may have different complex phases. Thus, the transformation matrix that brings 3 families of chiral multiplets $Q$ into the mass eigenstates can have complex phases, leading to the source of CP violation in the Cabbibo-Kobayashi-Maskawa matrix. The mechanism of generating Yukawa coupling in Type IIA string / M theory [54] is now generalized above so that it is valid even when $E_{n}$-type intersection of 2-cycles are involved in the $G_{2}$-holonomy manifold.

One thing that one immediately notices is that the diagonal entries of the up-type quark Yukawa matrix may be suppressed, rather than unsuppressed. This is because the sum of 


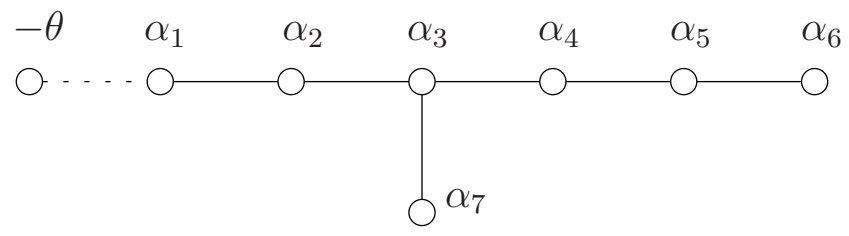

Figure 2: The (extended) Dynkin diagram of $E_{7}$. The $\mathfrak{s u}(2)_{2}$ is generated by $\alpha_{1}$, and $\mathfrak{s u}(6)_{1}$ by $\alpha_{3,4,5,6,7}\left(\mathfrak{s u}(5)_{\text {GUT }}\right.$ without $\left.\alpha_{7}\right)$. The highest root $\theta$ satisfies $-\theta+2 \alpha_{1}+3 \alpha_{2}+4 \alpha_{3}+3 \alpha_{4}+$ $2 \alpha_{5}+\alpha_{6}+2 \alpha_{7}=0$.

2-cycles $-C_{2}-C_{2}+\left(C_{1}+2 C_{2}\right)$ is not topologically trivial. Diagonal entries can be generated only by M2 branes that once sweep a broad region over the base manifold $Q$ so that discrete holonomy between $\vec{\zeta}^{2}(\vec{y})$ and $\vec{\zeta}^{1}(\vec{y})+\vec{\zeta}^{2}(\vec{y})$ of the 2 -fold cover can convert $C_{2}$ to $C_{1}+C_{2}$ or vice versa. Thus, when diagonal entries are generated, they are suppressed exponentially by the volume M2 branes swept. Here, we assume that the locus of enhanced gauge symmetry of $\left(C_{1}+2 C_{2}\right)$ is different from all of those of $C_{2}$ or $C_{1}+C_{2}$. Whether this feature can be consistent with the hierarchical and mixing pattern of quarks of the real world is left to a future investigation.

One can also attempt to connect our results to the ones of [55] where the $E_{6}$ was broken geometrically to $\mathrm{SU}(3)^{2} \times \mathrm{SU}(2)$. This will consist of further breaking of the GUT group $S U(5)$. We leave this for a further work.

\subsection{Minimal $E_{7}$ Model for All the Yukawa Couplings}

Let us now proceed to the $E_{7}$ model, where all the low-energy particles $\mathbf{1 0}, \overline{\mathbf{5}}, H(\mathbf{5})$ and $\bar{H}(\overline{\mathbf{5}})$ are obtained along with all the necessary Yukawa couplings. We consider a $G_{2}$ holonomy manifold $X$ as an ALE fibration over an associative 3-cycle $Q$ such that each ALE fibre space contains seven 2-cycles with $E_{7}$ intersection form. Four of the cycles, namely $C_{3}, C_{4}, C_{5}$, and $C_{6}$, remain intact over the entire $Q$, forming $A_{4}$ singularity and yield the $\mathrm{SU}(5)_{\text {GUT }}$ gauge fields. There should be no non-trivial holonomy flipping both ends of the $A_{4}$ Dynkin diagram of $\alpha_{3, \cdots, 6}$, as we discussed in section 4.1.

Over each point on $Q$, the ALE fibre is described by $\vec{\zeta}^{0,1,2,7}(\vec{y})$, which describe the "size" of the 2-cycles $C_{0,1,2,7}$. They obey the constraint

$$
\vec{\zeta}^{0}+2 \vec{\zeta}^{1}+3 \vec{\zeta}^{2}+2 \vec{\zeta}^{7}=0
$$

The two 2-cycles $C_{2}$ and $C_{1}+C_{2}$ are a doublet of the Weyl group $\mathfrak{S}_{2}^{(2)}$ of $\mathfrak{s u}(2)_{2}$, generated by $\alpha_{1}$ in the sense stated for the $E_{6}$ case. Chiral matters arise at points where some linear combi- 


\begin{tabular}{|c|c|c|c|c|c|} 
particles & $\mathbf{1 0}$ & $\overline{\mathbf{5}}$ & $H(\mathbf{5})$ & $\bar{H}(\overline{\mathbf{5}})$ & $\bar{N}$ \\
\hline 2-cycles & $-\left(C_{2}+C_{3}+C_{7}\right)$, & $C_{2}$, & $C_{1}+2 C_{2}+2 C_{7}$ & $C_{7}$ & $C_{-\theta}$, \\
& $-\left(C_{1}+C_{2}+C_{3}+C_{7}\right)$ & $\left(C_{1}+C_{2}\right)$ & $+3 C_{3}+2 C_{4}+C_{5}$ & & $C_{-\theta}+C_{1}$ \\
\hline singularity & $D_{5}$ & $A_{5}$ & $A_{5}$ & $A_{5}$ & $A_{1}+A_{4}$
\end{tabular}

Table 4: Correspondence between the low-energy particles and the 2-cycles on which M2brane are wrapped. The relevant 2-cycles are determined only up to adding some linear combinations of $C_{3}, C_{4}, C_{5}$, and $C_{6}$. For Higgs multiplets that are not doublets of SU(2) 2 , only one 2-cycle is shown, but there are four others, which can be read out from the appendix A. For other multiplets, which are $\mathrm{SU}(2)_{2}$ doublets, two 2-cycles are shown. The last row shows the enhanced singularity when the relevant 2-cycle shrinks. Note that the particle identification in this table is based on the particle identification pattern A in Table 1.

nations of those 2-cycles shrink, and singularity is enhanced. For different types of enhanced singularity and symmetry at different points on $Q$, we have different chiral multiplets.

The correspondence between the extra vanishing cycles and chiral matters can be understood by carefully following the symmetry breaking of $E_{7}$ down to $\mathrm{SU}(5)_{\mathrm{GUT}} \times \mathrm{SU}(2)_{2} \times \mathrm{U}(1)_{q_{6}} \times$ $\mathrm{U}(1)_{q_{7}}$, and the decomposition of the representations. This is carried out in the appendix A. and the results are shown in Table 4. As long as the loci of enhanced singularity (and gauge symmetry) are mutually isolated, the enhanced gauge symmetry is either $D_{5}$ or $A_{5}$, and the Type IIA interpretation that holds locally around each locus determines the multiplicity (just one) and the chirality depending on the sign of the Jacobian $|\partial \vec{\zeta}(\vec{y}) / \partial \vec{y}|$ around the intersection point of the relevant D6-branes (and O6-planes).

The trilinear Yukawa couplings appear when three M2 branes merge and disappear, in other words when the sum of the 2-cycles on which M2 branes are wrapped become topologically trivial. We can see that all the necessary Yukawa couplings are generated in that way:

$$
\begin{array}{rcl}
u \text {-Yukawa : } & \mathbf{1 0 . 1 0 . H ( 5 )} & -\left(C_{1}+C_{2}+C_{7}\right)-\left(C_{2}+C_{7}\right)+\left(C_{1}+2 C_{2}+C_{7}\right) \equiv 0, \\
d, e \text {-Yukawa: } & \overline{\mathbf{5}} \mathbf{1 0 .} \cdot \bar{H}(\overline{\mathbf{5}}) & C_{2}\left[+C_{1}\right]-\left(C_{2}+C_{7}\left[+C_{1}\right]\right)+C_{7} \equiv 0 \\
\nu \text {-Yukawa: } & \bar{N} \cdot \overline{\mathbf{5}} \cdot H(\mathbf{5}) & C_{-\theta}+\left(C_{1}+C_{2}\right)+\left(C_{1}+2 C_{2}+2 C_{7}\right) \equiv 0
\end{array}
$$

where the equalities hold mod $+C_{3,4,5,6}$; the last line is for the Dirac mass terms for neutrinos. On the other hand, the dangerous operators leading to proton decay are not generated by 
merging M2-branes, since the sums of relevant 2-cycles are not topologically trivial:

$$
\begin{array}{ccc}
\text { dimension-4 : } & \overline{\mathbf{5}} \mathbf{1 0 . \overline { \mathbf { 5 } }} & -\left(C_{2}+C_{7}\left[+C_{1}\right]\right)+2\left(C_{2}\left[+C_{1}\right]\right) \not \equiv 0, \\
\text { dimension-5 : } & \mathbf{1 0 . 1 0 . 1 0 . 5} & -3\left(C_{2}+C_{7}\left[+C_{1}\right]\right)+\left(C_{2}\left[+C_{1}\right]\right) \not \equiv 0 .
\end{array}
$$

In Heterotic theory, the existence of Yukawa couplings and absence of too rapid proton decay was controlled by the existences or not the trivial bundles in the tensor products of vector bundles for relevant particles. In M-theory compactification, the topological triviality of 2cycles replaces the bundle terminology. No matter which language is used, physical results should be the same. Particle physics does not depend so much on whether the vacuum is described by Heterotic theory or M-theory but by what the underlying symmetry is and how it is broken - the idea described in section 2. As we saw in section 2, $E_{7}$ is the minimal symmetry to accommodate all the low-energy particles, and there, both dimension-4 and dimension-5 proton decay operators are forbidden, while all the necessary Yukawa couplings are allowed. In the M-theory language, the $\mathrm{SU}(2)_{2}$ part is taken care by the $C_{2}$ and $C_{1}+C_{2}$ components and the $\mathrm{U}(1)$ part by the $C_{7}$ component, in the argument lead to the absence of proton decay.

Statements on the hierarchy and CP phases written for the up-type quark Yukawa couplings also hold for all the Yukawa matrices in (99)-(101). For vacua with M-theory (or Type IIA) description, chiral matters are localised at some points on a real 3-dimensional cycle in the M-theory (Type IIA) picture, and the Yukawa couplings are generated by spanning M2 branes (world sheets) between three points on the 3-cycle. This picture is certainly more intuitively tractable ${ }^{25}$ than calculating zero-mode wavefunctions on a Calabi-Yau complex 3 -fold with unknown metric. Since the minimal model presented here has known source of Yukawa matrices and is guaranteed to be free of dangerous proton decay, it deserves further investigation. ${ }^{26}$

\subsection{Local Model with $E_{8}$ Intersection Form}

The ALE geometry in the fibre allows chains of 2-cycles with $E_{8}$ intersection form so we can also consider M-theory compactification with the $E_{8}$ symmetry. The geometry of $E_{8}$ type

\footnotetext{
${ }^{25}$ This does not mean that the vacua with M-theory description are phenomenologically better than those without one.

${ }^{26}$ It should be kept in mind that the Wilson line on a quotient geometry obtained by freely acting symmetry cannot immediately solve the doublet-triplet splitting problem in this model, as noted in section 2
} 
ALE space is described by a set of data $\vec{\zeta}^{-\theta, 1,2,7,8}(\vec{y})$, satisfying

$$
\vec{\zeta}^{-\theta}+2 \vec{\zeta}^{1}+4 \vec{\zeta}^{2}+2 \vec{\zeta}^{7}+3 \vec{\zeta}^{8}=\overrightarrow{0} .
$$

The $\mathrm{SU}(5)_{\mathrm{GUT}}$ gauge field comes from vanishing 2-cycles $C_{3}, C_{4}, C_{5}$ and $C_{6}$. Chiral matters arise where some linear combinations of $C_{1}, C_{2}, C_{7}, C_{8}$ (and $C_{-\theta}$ ) shrinks and singularity (and gauge symmetry) is enhanced. It is a straightforward procedure to determine the vanishing 2-cycles corresponding to the low-energy multiplets. One can just read out the roots in (199)-(200) for the irreducible pieces identified with the low-energy particles in (119)-(21). We do not explicitly check whether the necessary Yukawa couplings are generated because our argument in section 2 guarantees it. The existence or absence of Yukawa couplings is just determined by $E_{8}$ algebra. The same is true for the dimension- 4 proton decay operators.

Let us take a closer look at the geometry. There are three different choices of vector bundles in the Heterotic compactifications, namely (22), (301) and (22) and the first two bundles are extensions of the last one. In the rest of this subsection, we clarify how the relations of these "bundles" are described in M-theory geometric terminology.

The $\mathrm{SU}(2)_{2} \times \mathrm{SU}(2)_{1} \times \mathrm{U}(1) \times \mathrm{U}(1)$-bundle compactification of Heterotic theory corresponds to the symmetry breaking pattern of removing the nodes $\alpha_{2}, \alpha_{7}$ and $\alpha_{8}$ from the extended Dynkin diagram in Fig. 3. The $\mathrm{SU}(2)_{2}$ and $\mathrm{SU}(2)_{1}$ are generated by $\alpha_{1}$ and $-\theta$, respectively. Their $\mathrm{SU}(2)$ bundles in Heterotic compactification corresponds to 2-fold covers of the data $\left(\vec{\zeta}^{2}(\vec{y}), \vec{\zeta}^{1}(\vec{y})+\vec{\zeta}^{2}(\vec{y})\right)$ - a doublet of the Weyl group $\mathfrak{S}_{2}^{(2)}$ of $\mathrm{SU}(2)_{2}$-and $\left(\vec{\zeta}^{7}(\vec{y})\right.$, $\vec{\zeta}^{7}(\vec{y})+\vec{\zeta}^{0}(\vec{y})$ ) - a doublet of the Weyl group $\mathfrak{S}_{2}^{(1)}$ of $\mathrm{SU}(2)_{1}$. The remaining $\vec{\zeta}^{8}$ is determined from the four others through (104) and the cycle $C_{8}$ is topologically equivalent to a certain linear combination of $C_{1}, C_{2}, C_{7}$ and $C_{-\theta}$. We reinterpret the geometry by thinking of another $\mathfrak{S}_{2}^{(1),(2)}$-singlet $C_{8}+\left(2 C_{7}+C_{-\theta}\right)=-\left(2 C_{8}+2 C_{1}+4 C_{2}\right)$ instead of $C_{8}$ because this choice of the 2-cycle will shortly turn out to be more convenient. The data are glued between two adjacent patches through

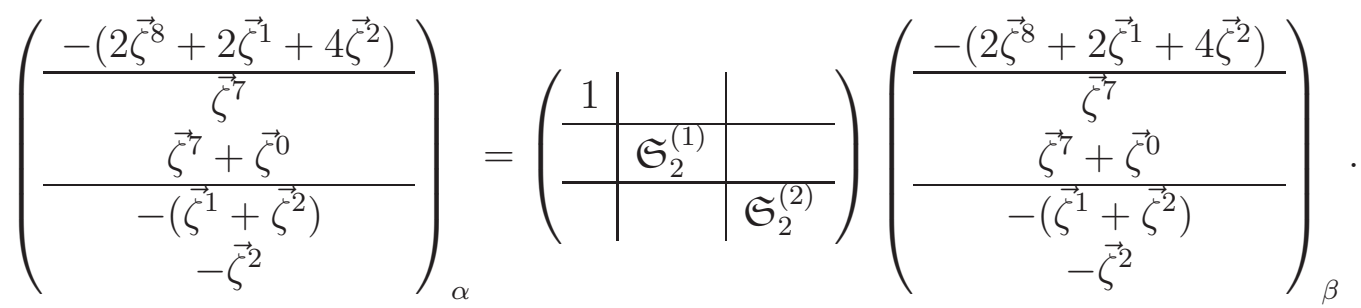

The $\mathrm{SU}(4) \times \mathrm{U}(1)_{\chi}$ bundle compactification of Heterotic theory corresponds to the symmetry breaking pattern of removing $\alpha_{7}$ in the extended Dynkin diagram Fig. 目 The SU(4) 


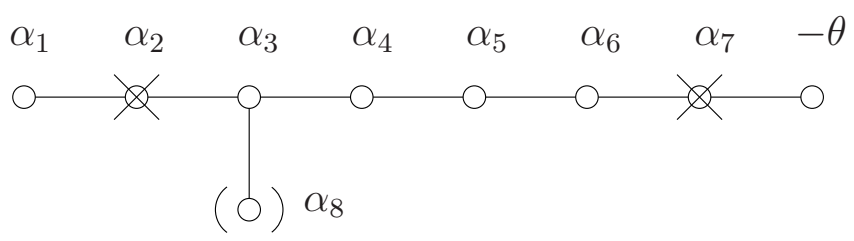

Figure 3: The extended Dynkin diagram of $E_{8}(\mathrm{I})$. The subalgebra $\mathfrak{s u}(2)_{2}+\mathfrak{s u}(2)_{1}+\mathfrak{s u}(6)_{1}$ is generated by the simple roots $\alpha_{1},-\theta$, and $\alpha_{3,4,5,6,7,8}$, respectively.

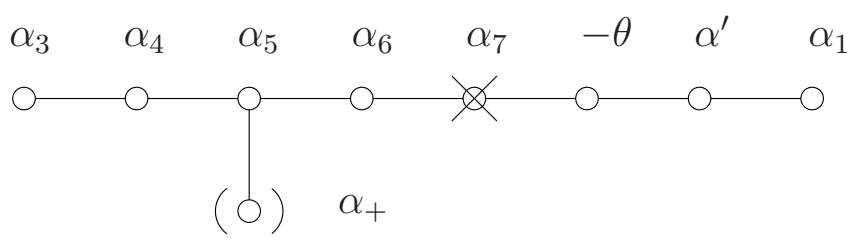

Figure 4: The extended Dynkin diagram of $E_{8}$ (II), and its maximal subalgebra $\mathfrak{s o}(10)+\mathfrak{s u}(4)$. $\alpha_{+} \equiv L_{0}-\left(L_{3}+L_{4}+L_{5}\right)$.

structure group of the vector bundle is generated by the simple roots $-\theta, \alpha^{\prime}$ and $\alpha_{1}$, where a root $\alpha^{\prime}$ is defined in the appendix A As the two rank- 2 vector bundles $\boldsymbol{2}_{1}$ and $\mathbf{2}_{2}$ are extended to a rank-4 bundle $U_{4}$ in Heterotic description, the two 2-fold cover data, or two doublets of 2-cycles $\left(C_{7}, C_{7}+C_{-\theta}\right)$ and $\left(C_{2}, C_{1}+C_{2}\right)$ should be paired up into a quartet in M-theory description; indeed, the quartet should be $\left(C_{7}, C_{7}+C_{-\theta}, C_{7}+C_{-\theta}+C_{\alpha^{\prime}}, C_{7}+C_{-\theta}+C_{\alpha^{\prime}}+C_{1}\right)$ and since

$$
\alpha_{7}+(-\theta)+\alpha^{\prime} \equiv-\left(\alpha_{1}+\alpha_{2}\right)
$$

$\bmod \alpha_{3,4,5,6}$, it is the quartet $\left(C_{7}, C_{7}+C_{-\theta},-\left(C_{1}+C_{2}\right),-C_{2}\right)$ that mix together over the entire base manifold $Q$. The data $\left(\vec{\zeta}^{7}, \vec{\zeta}^{7}+\vec{\zeta}^{-\theta},-\left(\vec{\zeta}^{1}+\vec{\zeta}^{2}\right),-\vec{\zeta}^{2}\right)$ is a 4 -fold cover of $Q$. Note that the other 2-cycle $-\left(2 C_{8}+2 C_{1}+4 C_{2}\right)$ is $C_{7}-C_{\alpha^{\prime \prime}}$, where the root $\alpha^{\prime \prime}$ is defined in the appendix A. The transition function in (105) is enlarged into $\mathbf{1} \times \mathfrak{S}_{4}$.

The $\mathrm{SU}(3)_{2} \times \mathrm{SU}(2)_{2} \times \mathrm{U}(1)_{\tilde{q}_{7}}$ bundle compactification of Heterotic theory corresponds to the symmetry breaking pattern of removing the node $\alpha_{2}$ in the extended Dynkin diagram in Fig. 5.5 The replacing the bundle $1 \oplus \mathbf{2}_{1}$ by $U_{3}[(\underline{22})$ by $[\underline{30})]$ in the Heterotic side corresponds to mixing up the 2-cycle $-\left(2 C_{8}+2 C_{1}+4 C_{2}\right) \equiv C_{7}-C_{\alpha^{\prime \prime}}$ with the $\mathfrak{S}_{2}^{(1)} \operatorname{doublet}\left(C_{7}, C_{7}+C_{-\theta}\right)$ globally on $Q$ to be a triplet of the Weyl group $\mathfrak{S}_{3}$ generated by $\alpha^{\prime \prime}$ and $-\theta$. The data $\left(-\left(2 \vec{\zeta}^{8}+2 \vec{\zeta}^{1}+4 \vec{\zeta}^{2}\right), \vec{\zeta}^{7}, \vec{\zeta}^{7}+\vec{\zeta}^{-\theta}\right)$ gives a 3 -fold cover of $Q$. The $\mathfrak{S}_{2}^{(2)}$-doublet $\left(-\left(C_{1}+C_{2}\right),-C_{2}\right)$ remains intact in the process of lifting the " $2+2+1$ bundles" to " $3+2$ bundles." The transition function in (105) is enlarged into $\mathfrak{S}_{3} \times \mathfrak{S}_{2}^{(2)}$. 


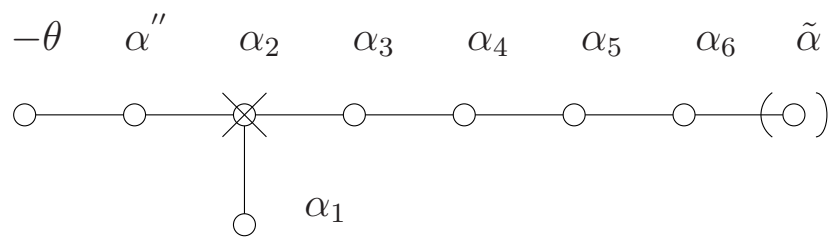

Figure 5: Extended Dynkin diagram of $E_{8}(\mathrm{III})$, and its maximal subalgebra $\mathfrak{s u}(3)_{2}+\mathfrak{s u}(2)_{2}+$ $\mathfrak{s u}(6)_{2} . \tilde{\alpha} \equiv 2 L_{0}-\left(L_{1}+\cdots+L_{6}\right)$.

The quintet data $\left(-\left(2 \vec{\zeta}^{8}+2 \vec{\zeta}^{1}+4 \vec{\zeta}^{2}\right), \vec{\zeta}^{7}, \vec{\zeta}^{7}+\vec{\zeta}^{-\theta},-\left(\vec{\zeta}^{1}+\vec{\zeta}^{2}\right),-\vec{\zeta}^{2}\right)$ are mutually separated by

$$
\begin{array}{rcc}
\vec{\zeta}^{\alpha \prime} & \equiv & 2 \vec{\zeta}^{1}+4 \vec{\zeta}^{2}+\vec{\zeta}^{7}+2 \vec{\zeta}^{8} \\
& \vec{\zeta}-\theta & \\
\vec{\zeta}^{\alpha} & \equiv & \vec{\zeta}^{1}+3 \vec{\zeta}^{2}+\vec{\zeta}^{7}+3 \vec{\zeta}^{8} \\
& \overrightarrow{\zeta^{1}}, & \\
\vec{\zeta}^{\alpha \prime \prime} & \equiv & -\left(2 \vec{\zeta}^{1}+3 \vec{\zeta}^{2}+2 \vec{\zeta}^{8}\right) .
\end{array}
$$

Since these separations are defined in a cyclic order, they satisfy a constraint

$$
\vec{\zeta}^{\prime \prime}+\vec{\zeta}^{-\theta}+\vec{\zeta}^{\alpha^{\prime}}+\vec{\zeta}^{1}+\vec{\zeta}^{\prime \prime \prime}=0
$$

which is equivalent to the constraint (104), and is also in the form of the traceless condition for the $A_{4}$ type ALE space [80, corresponding to the $\mathrm{SU}(5)$ structure group of the Heterotic description. Irreducible vector bundles with full SU(5) structure group correspond to irreducible 5 -fold cover of $Q$ given by the quintet data in the M-theory description. Following the idea in section 2, we suggest that the absence of dimension 4 proton decay is an indication that the 5 -fold cover is not irreducible at some level of approximation; it is split up into irreducible components of either (2-fold, 2-fold, single) covers, (4-fold, single) covers or (3-fold, 2-fold) covers.

We have seen at the end of section 4.1 that the diagonal entries of up-type quark Yukawa matrix tend to be suppressed. The argument there does not depend specifically on the fact that $\left(\vec{\zeta}^{2}, \vec{\zeta}^{1}+\vec{\zeta}^{2}\right)$ is a $\underline{2 \text {-fold }}$ cover; rather the argument is more general, and is valid also in the case of 4 -fold cover. Thus, the suppressed diagonal entries can be regarded as generic predictions of M-theory vacua with $\mathrm{SU}(5)_{\text {GUT }}$ unification. 


\section{F-theory Vacua}

The Heterotic theory compactified on $T^{2}$ has the same moduli space as that of the F-theory on elliptic K3 [16]. Likewise, the Heterotic theory on elliptic Calabi-Yau 3-fold

$$
\pi_{Z}: Z \rightarrow B
$$

has the same moduli space ${ }^{27}$ as that of the F-theory on elliptic Calabi-Yau 4-fold

$$
\pi_{X}: X \rightarrow B^{\prime}
$$

whose base manifold $B^{\prime}$ is a $\mathbb{P}^{1}$-fibration on the common $B$,

$$
\pi^{\prime \prime}: B^{\prime} \rightarrow B
$$

so that

$$
\pi^{\prime}: X \rightarrow B
$$

is a K3-fibration [17, 18, 19, 20].

For vacua that fall into the category of Heterotic-F-theory dual, some region of the moduli space is described better by the Heterotic theory but other regions are better described by F-theory. Furthermore, there will be vacua that do not fall into the shared moduli space, and in particular, some of F-theory compactification on a non-K3-fibred elliptic Calabi-Yau 4-fold may not have Heterotic descriptions. Therefore, in this section, we take a step toward translating what has been discussed in earlier sections into F-theory language, so that we get prepared for exploring vacua that are well-described by the F-theory.

\subsection{Trilinear Yukawa Couplings}

One way to see the Heterotic-F-theory duality is through the del Pezzo fibration. Vector bundles on an elliptic Calabi-Yau 3-fold $\pi: Z \rightarrow B$ with the structure group in $E_{8}$ are described $^{28}$ by $d P_{8}$-fibration $W \rightarrow B$. One point in $d P_{8}$ fibre is blown up to be $d P_{9}$, and the $d P_{9}$ fibres for the visible and hidden sectors add up to form the elliptic $K 3$ fibration $\pi^{\prime}: X \rightarrow B$ of the F-theory [19].

When we discuss Yukawa couplings in the visible sector, we do not pay attention to the del Pezzo fibration for the hidden sector. The commutation relation of $E_{8}$ that determines

\footnotetext{
${ }^{27}$ The overlap between Heterotic vacua and F-theory vacua is more than this. See, for example, [18, [56] [57].

${ }^{28}$ To be more precise, not all the information is encoded in the del Pezzo fibration. Discrete topological choices that changes the chirality are one example.
} 
Yang-Mills interaction of the Heterotic theory is now encoded in the intersection form of the 2-cycles of the $d P_{8}$ ( or $d P_{9}$ ). Those surfaces contain the chains of 2-cycles that we discussed in section 4 and the identifications of particles and interactions there can also be valid for the F-theory. Thus, the $E_{8}$ version of the discussion around (99)-(103) is also valid in the F-theory vacua. As is clear from the discussion in section 4 , the existence of trilinear Yukawa couplings and absence of dangerous proton decay operators can be discussed in that way, as long as the local geometry in $X$ around the holomorphic 4-cycle of the visible sector gauge group has the fibration of eight (or seven) 2-cycles with the right intersection forms. The validity of this way of thinking is not limited to F-theory vacua that have Heterotic dual.

\section{$\mathbf{5 . 2}$ F-theory Dual of Heterotic $E_{8} / \mathrm{SU}(5)_{\mathrm{GUT}} \times\left\langle\mathrm{SU}(4) \times \mathrm{U}(1)_{\chi}\right\rangle$ Model}

If one is only interested in knowing whether Yukawa couplings exist or proton decay operators are absent, there is no essential difference between the Heterotic, M and F-theory. Such aspects were determined by the underlying symmetry such as $E_{7}$ or $E_{8}$ and how it is broken. The above argument is fine for F-theory vacua. But when one wants to extract more physical consequences of F-theory vacua, we need more detailed descriptions. For that purpose, we begin with translating the Heterotic models discussed in section 3 into F-theory language through the Heterotic-F-theory duality. Section 5.2 is devoted to the translation of $E_{8} / \mathrm{SU}(5)_{\text {GUT }} \times$ $\left\langle\mathrm{SU}(4) \times \mathrm{U}(1)_{\chi}\right\rangle$ model, and section 5.3 to $E_{8} / \mathrm{SU}(5)_{\mathrm{GUT}} \times\left\langle\mathrm{SU}(3)_{2} \times \mathrm{SU}(2)_{2} \times \mathrm{U}(1)_{\tilde{q}_{7}}\right\rangle$ and $E_{8} / \mathrm{SU}(5)_{\mathrm{GUT}} \times\left\langle\mathrm{SU}(2)_{1} \times \mathrm{SU}(2)_{2} \times \mathrm{U}(1) \times \mathrm{U}(1)\right\rangle$ models. Later in section 5.4, we discussed how various descriptions obtained through translation can be generalized to F-theory vacua that may not have Heterotic dual.

\subsubsection{Matter Curves at the Reducible Limit}

An intriguing aspect of F-theory vacua is that charged matters are localized in complex curves in the internal space $B^{\prime}[19,26$. When one wants to have better understanding of the pattern of Yukawa matrices, for instance, it is crucial to know the wavefunctions of the chiral zero modes. Thus the determination of the matter curve is an important issue. Although the localisation pattern of matters in $\mathrm{SU}(5)_{\text {GUT }}$ unbroken F-theory vacua is well-known in the literature, we repeat this separately for the case where the structure group of the bundle is reduced to $\mathrm{SU}(4) \times \mathrm{U}(1)_{\chi}$ subgroup of $\mathrm{SU}(5)$. Although the unbroken gauge symmetry is the same $\mathrm{SU}(5)_{\mathrm{GUT}}$, we see the matter curves are not of the generic $\mathrm{SU}(5)$ unified theories.

The rank- $n$ vector bundles on an elliptic Calabi-Yau 3-fold $\pi: Z \rightarrow B$ for Heterotic 
compactification are constructed out of a set of data $(C, \mathcal{N})$, where $C \subset Z$ is a $n$-fold spectral cover $\pi_{C}: C \rightarrow B$ and $\mathcal{N}$ is a line bundle on $C$. A bundle for $(C, \mathcal{N})$ is given by

$$
V=p_{2 *}\left(p_{1}^{*}(\mathcal{N}) \otimes \mathcal{P}_{B}\right)
$$

where $p_{1,2}$ are maps associated with a fibre product

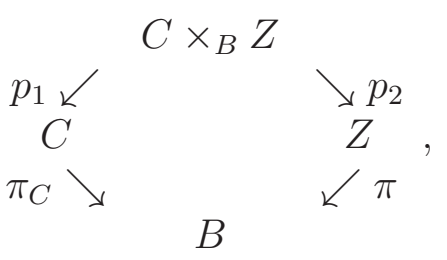

$q \equiv \pi_{C} \circ p_{1}=\pi \circ p_{2}$, and $\mathcal{P}_{B}$ is the Poincare line bundle $\mathcal{O}_{C \times_{B} Z}\left(\Delta-\sigma_{1}-\sigma_{2}+q^{*} K_{B}\right)[19]$. The first Chern class of the vector bundle $V$ is given by

$$
c_{1}(V)=\pi_{C *}\left(c_{1}(\mathcal{N})-\frac{1}{2} r\right)
$$

where $r \equiv \omega_{C / B} \equiv K_{C}-\pi_{C}^{*} K_{B}$ is the ramification divisor on $C$ of $\pi_{C}: C \rightarrow B$ [58], and $c_{1}(V)$ is a 2 -form on the base 2-fold $B$. When one thinks of a bundle with the structure group $\mathrm{U}(n)$, rather than $\mathrm{SU}(n)$, [119]) does not have to be zero [59]. The second Chern character is

$$
\operatorname{ch}_{2}(V)=-\sigma \cdot \eta+\pi^{*} \omega,
$$

where $\sigma$ is a section $\sigma: B \rightarrow Z$ of the elliptic fibration, $\eta$ is a divisor on $B$ that determines the $n$-fold spectral cover $C \sim n \sigma+\eta$, and $\omega$ is some 2 -form on $B$.

When the line bundle $L$ of (29) on an elliptic Calabi-Yau 3-fold is given by spectral data $\left(C_{1}, \mathcal{N}_{1}\right)$, the spectral cover $C_{1}$ is just $\sigma$. Indeed, if it had a component of a divisor $\eta_{1}$ on $B$, then $\operatorname{ch}_{2}(L)$ of the line bundle $L$ would have a component $\sigma \cdot \eta_{1}$ that is not strictly on the base $B$ despite $c_{1}(L)$ strictly on the base [59]. Thus, $C_{1} \sim \sigma$. The line bundle $\mathcal{N}_{1}$ on $\sigma \sim B$ is identified with the line bundle $L$ itself. We abuse the notation a little bit hereafter, and $L$ stands for both the line bundle on $B$ and $Z$.

Let the bundle $U_{4}$ be given by the data $\left(C_{4}, \mathcal{N}_{4}\right)$, and $C_{4} \sim 4 \sigma+\eta_{4}$. Then the data for 
the bundles in various representations are given by

$$
\begin{array}{rcl}
U_{4} & \text { for } \mathbf{1 0} & \left(4 \sigma+\eta_{4}, \mathcal{N}_{4}\right), \\
L & \mathbf{1 0}^{\prime} \text { absent in LE. } & (\sigma, L), \\
\wedge^{2} U_{4} & \text { for } \bar{H}(\overline{\mathbf{5}}) \text { and } H(\mathbf{5})^{\dagger} & \left(6 \sigma+2 \eta_{4}, \mathcal{N}_{\wedge^{2} U_{4}}\right), \\
U_{4} \otimes L & \text { for } \overline{\mathbf{5}} & \left(4 \sigma+\eta_{4}, \mathcal{N}_{4} \otimes L\right), \\
U_{4} \otimes L^{-1} & \text { for } \bar{N} & \left(4 \sigma+\eta_{4}, \mathcal{N}_{4} \otimes L^{-1}\right) .
\end{array}
$$

Equation (123) is due to

$$
C_{\wedge^{2} V_{r}} \sim \frac{r(r-1)}{2} \sigma+(r-2) \eta_{r}
$$

for the anti-symmetric representation of a rank- $r$ bundle $V_{r}$ with a spectral surface $C_{r} \sim$ $r \sigma+\eta_{r}$ 34. Note that the sum of the spectral cover of $U_{4}$ and $L$ is $C_{V_{5}} \sim 5 \sigma+\eta_{4}$, and that of $\wedge^{2} U_{4}$ and $U_{4} \otimes L$ is $C_{\wedge^{2} V_{5}} \sim 10 \sigma+3 \eta_{4}$. The reducible limit of the vector bundle (29) corresponds to the case where the spectral surfaces of the $\mathrm{SU}(5)$ bundle become reducible, and $C_{5} \sim 5 K_{B}+\eta_{4}$ splits into irreducible pieces $C_{4} \sim 4 K_{B}+\eta_{4}$ and $C_{1} \sim \sigma[60$.

In Heterotic theory, the cohomology of the vector bundles, and hence massless modes, can be calculated by the spectral sequence, evaluating the cohomology in the elliptic fibre direction first, and in the base manifold later. For a bundle $\rho(V)$, the cohomology vanishes only over a curve $\bar{c}_{\rho(V)} \equiv C_{\rho(V)} \cdot \sigma$ on the base $B$ [19, 61]. ${ }^{29}$ Thus, the massless matters are localised on curves on $B$ given by

$$
\begin{aligned}
\bar{c}_{\mathbf{1 0}}=\bar{c}_{\overline{\mathbf{5}}}=\bar{c}_{\bar{N}} & =4 K_{B}+\eta_{4} \equiv \bar{c}_{\mathbf{1 6}} \\
\bar{c}_{H, \bar{H}} & =6 K_{B}+2 \eta_{4}+\bar{c}_{\mathbf{v e c} .}, \\
\left(\bar{c}_{\mathbf{1 0}}\right. & \left.=K_{B}, \quad \text { spread out in } B\right)
\end{aligned}
$$

where $\sigma \cdot \sigma \sim \sigma \cdot K_{B}[19]$ was used.

In equation (129) we see that the fields $\overline{5}$ and $H$ have different localisation. Therefore the argument of the existence of the Yukawa couplings and absence of the proton decay holds well to F-theory picture.

The matter curves $\bar{c}_{\mathbf{1 0}}=5 K_{B}+\eta_{4}$ for $\mathrm{SU}(5)_{\mathrm{GUT}} \mathbf{- 1 0}$ representation and $\bar{c}_{\overline{\mathbf{5}}}=10 K_{B}+3 \eta_{4}$ for $\mathrm{SU}(5)_{\mathrm{GUT}^{-}} \overline{\mathbf{5}}$ representation split into irreducible pieces. Although $(Q, \bar{U}, \bar{E}),(\bar{D}, L)$ and

\footnotetext{
${ }^{29}$ This localization is is not an artifact in calculation, but is indeed physical, when the volume of the $T^{2}$ fibre in the Heterotic compactification is not large. Such region of the Heterotic moduli space is better described by the F-theory, where matters are expected to localised.
} 
$\bar{N}$ originate from different irreducible bundles, their matter curves are the same, as in the case of $\mathrm{SU}(4)$-bundle compactification with unbroken $\mathrm{SO}(10)$ symmetry. This is because $L$ and $\operatorname{det} U_{4}$ are line bundles only on the base manifold $B$ and the only source of the distinction among 10, $\overline{\mathbf{5}}$ and $\bar{N}$ representations is the twist of those two bundles. Their effects come in only on the matter curves $\bar{c}_{\mathbf{1 6}}$ and not at the level of changing the spectral surfaces or matter curves themselves.

In the F-theory dual of these Heterotic vacua obtained in (114)-(116), and the $\mathbb{P}^{1}$ fibration (115) is such that the $z=0$ section of (115) $\Sigma_{0} \subset B^{\prime}\left(\Sigma_{0}\right.$ is isomorphic to $\left.B\right)$ satisfies [17, 18, 19, 20, 157]:

$$
\Sigma_{0} \cdot \Sigma_{0} \sim\left(6 K_{B}+\eta_{4}\right)
$$

The divisor $\Sigma_{0}$ of $B^{\prime}$ is where the unbroken SU $(5)_{\text {GUT }}$ gauge field propagates in F-theory. Over $\Sigma_{0}$ there are loci of enhanced singularity and enhanced gauge symmetry, and this is where matters are localised, and they are the same as the matter curves obtained in the Heterotic argument above [19, 26]. The reducible limit of vector bundles in Heterotic theory correspond to taking some coefficients of the polynomial defining (114) to zero. The coefficients for SU(5) bundles are given by global holomorphic sections $g \in H^{0}(B, \mathcal{O}(\eta)), f \in H^{0}\left(B, \mathcal{O}\left(2 K_{B}+\eta\right)\right)$, $q \in H^{0}\left(B, \mathcal{O}\left(3 K_{B}+\eta\right)\right), H \in H^{0}\left(B, \mathcal{O}\left(4 K_{B}+\eta\right)\right)$ and $h \in H^{0}\left(B, \mathcal{O}\left(5 K_{B}+\eta\right)\right)$ [26], and the dual of $\mathrm{SU}(4) \times \mathrm{U}(1)_{\chi}$ bundle with $m=0$ in (143) corresponds to taking $h=0$. The loci of enhanced singularity $\bar{c}_{\mathbf{1 0}}$ and $\bar{c}_{\overline{5}}$ rearrange themselves in this limit, so that they are grouped as $\bar{c}_{\mathbf{1 6}}, \bar{c}_{\text {vec. }}$ while the irreducible piece (129) supplements the roots in $\mathfrak{s o}(10) / \mathfrak{s u}(5)$, so that the locus $\Sigma_{0}$ support $\mathrm{SO}(10)$ gauge group.

The rearrangement argument of the matter curves still misses one piece: where has the $\mathrm{SU}(5)_{\text {GUT }}$-singlet part of the $\mathrm{SO}(10)-\mathbf{1 6}$ representation come from? This piece is for the right-handed neutrinos, and hence it should be related to the vector bundle moduli of SU(5) bundle that are set to zero in the reducible limit - in Heterotic language. Thus, it corresponds to $h \in H^{0}\left(B, \mathcal{O}\left(5 K_{B}+\eta_{4}\right)\right)$ in F-theory language. Those degrees of freedom are essentially localised on a curve defined by the intersection $C_{4} \cdot C_{1} \sim C_{4} \cdot \sigma$, which is also the definition of the $\bar{c}_{\mathbf{1 6}}$. This is because $h$ describes the reconnection of two irreducible pieces $C_{4}$ and $C_{1}$. The other way to see the localisation on $\bar{c}_{\mathbf{1 6}}$ is to use the exact sequence

$$
0 \rightarrow \mathcal{O}_{B}\left(C_{5}-C_{4}\right) \rightarrow \mathcal{O}_{B}\left(C_{5}\right) \rightarrow \mathcal{O}_{\sigma \cdot C_{4}}\left(C_{5}\right) \rightarrow 0
$$

Note that $C_{5}-C_{4} \sim \sigma \sim K_{B}$ on $B$. Since $h^{0}\left(B, \mathcal{O}\left(K_{B}\right)\right)=h^{2}\left(B, \mathcal{O}_{B}\right)$ and $h^{1}\left(B, \mathcal{O}\left(K_{B}\right)\right)=$ $h^{1}\left(B, \mathcal{O}_{B}\right)$ are zero in elliptic fibration (113) that leaves only $\mathcal{N}=1$ supersymmetry [62] we 
see that

$$
H^{0}\left(B, \mathcal{O}\left(5 K_{B}+\eta_{4}\right)\right) \simeq H^{0}\left(\bar{c}_{\mathbf{1 6}}, \mathcal{O}\left(5 K_{B}+\eta_{4}\right)\right) .
$$

Thus, $h \in H^{0}\left(B, \mathcal{O}\left(5 K_{B}+\eta_{4}\right)\right)$ is associated with a global holomorphic section of a sheaf on $\overline{\mathbf{c}}_{16}$.

$\mathrm{U}(1)_{\chi}$ bundle $L$ is turned on on the discriminant locus $\Sigma_{0} \simeq B$, and the $\mathrm{SO}(10)$ gauge symmetry is broken down to $\mathrm{SU}(5)_{\text {GUT }}$. The Fayet-Iliopoulos parameter due to U(1) flux itself is calculated in 63 in Type IIB string theory orientifold compactification, and although F-theory is not exactly the same as the Type IIB theory, it may be roughly the same. In Type IIB calculation [63] it is

$$
\xi \approx l_{s}^{-4} \int c_{1}(L) \wedge J
$$

after restoring proper dimensionality; numerical factors being ignored. This Fayet-Iliopoulos parameter may vanish when $c_{1}(L)$ is orthogonal to the Kähler form $J$ on the discriminant locus $\Sigma_{0} \simeq B$. In this case, the global $\mathrm{U}(1)_{\chi}$ symmetry is left unbroken, and the $\mathrm{U}(1)_{\chi}$ gauge boson is massive, due to the generalized Green-Schwarz interactions. If this FayetIliopoulos parameter is non-zero, the $\mathrm{U}(1)_{\chi}$-symmetry breaking phase transition is triggered and this symmetry is broken. Because of the same reason as in section 3 , either one of the sign of $\int_{\Sigma_{0}} c_{1}(L) \wedge J$ is phenomenologically acceptable, because otherwise $\overline{\mathbf{5}}$ multiplets are no longer distinguished from the $\bar{H}(\overline{\mathbf{5}})$ multiplet, and the dimension-4 proton decay operators are generated.

\subsubsection{Chiral Matter}

The massless modes $H^{1}(Z ; \rho(V))$ from vector bundles $\rho(V)$ of Heterotic theory can be obtained by cohomology on matter curves $\bar{c}_{\rho(V)}$, when we consider elliptic fibred Calabi-Yau 3-fold $Z \rightarrow B$ [19, 61]:

$$
H^{1}(Z ; \rho(V)) \simeq H^{0}\left(\bar{c}_{\rho(V)} ; \mathcal{F}_{\rho(V)}\right)
$$

where $\mathcal{F}$ is a sheaf on the matter curve $\bar{c}_{\rho(V)}$ such that $R^{1} \pi_{*} \rho(V)=i_{\bar{c}_{\rho(V)} *} \mathcal{F}$ for $i_{\bar{c}_{\rho(V)}}: \bar{c}_{\rho(V)} \rightarrow$ $B$; in particular, it is given in terms of spectral cover data by

$$
\mathcal{F}=j^{*} \mathcal{N}_{\rho(V)} \otimes i^{*} K_{B}
$$

where $j: \bar{c}_{\rho(V)} \rightarrow C_{\rho(V)}$. The net chirality (34)-(38) can be expressed as Euler characteristic on the matter curves as well 61].

$$
\begin{aligned}
\chi\left(R_{5}\right)=-\chi(Z, \rho(V)) & =h^{1}(Z, \rho(V))-h^{2}(Z, \rho(V)) \\
& =h^{0}\left(\bar{c}_{\rho(V)}, \mathcal{F}\right)-h^{1}\left(\bar{c}_{\rho(V)}, \mathcal{F}\right)=\chi\left(\bar{c}_{\rho(V)}, \mathcal{F}\right) .
\end{aligned}
$$


All above is valid as long as $c_{1}(\rho(V))$ is trivial in the fibre direction, and hence applicable to all the irreducible bundles considered in section 3.1. The net chirality of matters can be calculated on the matter curves. Since they are localised on the matter curves in F-theory vacua, there should be such expressions formulated on the matter curves; the net chirality does not depend on the global geometry of the elliptic Calabi-Yau 4-fold $X$ but only on geometric information along the matter curves.

The net chirality of quarks, leptons and right-handed neutrinos are given by

$$
\begin{aligned}
\# \bar{N}-\# \overline{\bar{N}} & =h^{0}\left(\bar{c}_{\mathbf{1}} ; \mathcal{O}\left(K_{\bar{c}_{\mathbf{1}}}\right)^{1 / 2} \otimes \mathcal{L}_{\gamma} \otimes L^{-1}\right)-h^{0}\left(\bar{c}_{\mathbf{1} \mathbf{6}} ; \mathcal{O}\left(K_{\bar{c}_{\mathbf{1}}}\right)^{1 / 2} \otimes \mathcal{L}_{\gamma}^{-1} \otimes L\right) \\
& =\int_{\bar{c}_{\mathbf{1}}} j^{*} \gamma-c_{1}(L), \\
\# \mathbf{1 0}-\# \overline{\mathbf{1 0}} & =h^{0}\left(\bar{c}_{\mathbf{1 6}} ; \mathcal{O}\left(K_{\bar{c}_{\mathbf{1}}}\right)^{1 / 2} \otimes \mathcal{L}_{\gamma}\right)-h^{0}\left(\bar{c}_{\mathbf{1 6}} ; \mathcal{O}\left(K_{\bar{c}_{\mathbf{1}}}\right)^{1 / 2} \otimes \mathcal{L}_{\gamma}^{-1}\right) \\
& =\int_{\bar{c}_{16}} j^{*} \gamma \\
\# \overline{\mathbf{5}}-\# \overline{\mathbf{5}} & =h^{0}\left(\bar{c}_{\mathbf{1 6}} ; \mathcal{O}\left(K_{\bar{c}_{\mathbf{1}}}\right)^{1 / 2} \otimes \mathcal{L}_{\gamma} \otimes L\right)-h^{0}\left(\bar{c}_{\mathbf{1 6}} ; \mathcal{O}\left(K_{\bar{c}_{\mathbf{1}}}\right)^{1 / 2} \otimes \mathcal{L}_{\gamma}^{-1} \otimes L^{-1}\right) \\
& =\int_{\bar{c}_{\mathbf{1}}} j^{*} \gamma+c_{1}(L),
\end{aligned}
$$

where a 2-form $\gamma$ on $C_{4}$ determines $c_{1}\left(\mathcal{N}_{4}\right)$ by

$$
c_{1}\left(\mathcal{N}_{4}\right)=\frac{1}{2} r+\gamma
$$

and $\mathcal{L}_{\gamma}$ is a line bundle on $\bar{c}_{16}$ whose first Chern class is $j^{*} \gamma$. In derivation of (137)-(139), we have used Hirzebruch-Riemann-Roch theorem $[36]^{30}$ and

$$
\frac{1}{2} j^{*}\left(K_{C_{\rho(V)}}-\pi^{*} K_{B}\right)+i^{*} K_{B}=\frac{1}{2} K_{\bar{c}_{\rho(V)}}=-\operatorname{td}_{1}\left(T \bar{c}_{\rho(V)}\right) .
$$

One could also separate the $\operatorname{det} U_{4}$ piece and set $j^{*} \gamma=j^{*} \gamma^{\prime}-c_{1}(L) / 4$; then

$$
\chi=\int_{\bar{c}_{16}}\left(j^{*} \gamma^{\prime}+\frac{q_{\chi}}{4} c_{1}(L)\right) .
$$

$\gamma$ is translated into the primitive $G^{(2,2)}$ flux in F-theory language [66]. The phenomenological constraint (42) reduces to ${ }^{31} c_{1}(T B) \cdot c_{1}(L)=-K_{B} \cdot c_{1}(L)=0$, and (43) to $\eta_{4} \cdot c_{1}(L)=0$ in elliptic Calabi-Yau compactification with $m=0$ in (143). Thus, $c_{1}(L)$ does not contribute to the net chirality of any of quarks, leptons and right-handed neutrinos.

\footnotetext{
${ }^{30}$ One has to pay more attention to whether the theorem is applicable in each explicit example. Here, we only deal with situations that do not require special treatment.

${ }^{31}$ Note that $c_{2}(T Z)=\sigma \cdot 12 c_{1}(T B)+\cdots$, where ellipses stand for the fibre class. $c_{1}(L)^{3}=0$ because $c_{1}(L)$ is purely on $B$. Note also that $c_{2}\left(U_{4}\right)=\sigma \cdot \eta_{4}+\cdots$.
} 


\subsubsection{Approach from Parabolic (Extension) Construction}

The spectral cover construction can only describe vector bundles with the first Chern class purely on the base manifold. This is reasonable because the $n$-point fibre of an $n$-fold spectral cover describes $n$ Wilson lines in the fibre direction, not a non-trivial field strength.

Purely from the viewpoint of Heterotic compactification, however, there seems to be no problem in considering a line bundle $L$ whose first Chern class has a fibre component:

$$
c_{1}(L)=m \sigma+\pi^{*} \omega_{1},
$$

where $\omega_{1}$ is a divisor on $B$ and $m$ an integer, and indeed, such compactification was discussed in [35, 64. Our discussion in section 3 does not exclude this possibility, either. However, when we consider a small fibre limit, the tree-level Fayet-Iliopoulos parameter is approximated by

$$
\xi_{\chi} \approx M_{G}^{2} l_{s}^{2} \frac{m \int_{B} J \wedge J}{\int_{Z} J \wedge J \wedge J} \approx m M_{G}^{2}\left(\frac{l_{s}^{2}}{\operatorname{vol} .\left(T^{2}\right)}\right),
$$

and cannot vanish when $m \neq 0$. Either $\bar{N}$ or $\bar{N}$ develops an expectation value, as we saw in section 3.1.2 and the vector bundle cannot remain reducible. Thus, in the small fibre limit, we cannot consider the reducible limit in Heterotic compactification if $m \neq 0$. In the construction of the moduli space of vector bundles in [19, such a reducible limit with $m \neq 0$ was cut out from the beginning. The moduli of spectral cover construction provides the description of vector bundles obtained after the $\mathrm{U}(1)_{\chi}$ breaking phase transition [19].

The absence of dimension- 4 proton decay requires that $\int_{Z} c_{1}(L) \wedge J \wedge J<0$, or $\xi_{\chi}<0$, assuming $N_{\text {gen }}>0$. On elliptic-fibred Calabi-Yau 3-folds, this condition simplifies; $m<0$ (or $m=0$ and $\xi<0$ in (133)).

Let us consider the simplest case, $m=-1$. In particular, when the rank- 4 bundle $U_{4}$ is $\mathcal{W}_{4} \otimes \mathcal{M}$ with $\mathcal{W}_{4}$ constructed in [19, 65] and a line bundle $\mathcal{M}$ on $B$, and $L \simeq \mathcal{O}(-\sigma+$ $\left.6 K_{B}\right) \otimes \mathcal{M}^{-4}$ the $\mathrm{U}(1)_{\chi}$ breaking phase transition is just the extension of $U_{4} \simeq \mathcal{W}_{4} \otimes \mathcal{M}$ by $\mathcal{W}_{1} \otimes \mathcal{O}\left(6 K_{B}\right) \otimes \mathcal{M}^{-4}$. In the fibration structure of the vector bundle moduli [19]

$$
0 \rightarrow H^{1}\left(B ; R^{0} \pi_{*} \operatorname{adj} .\left(V_{5}\right)\right) \rightarrow H^{1}\left(Z ; \operatorname{adj} .\left(V_{5}\right)\right) \rightarrow H^{0}\left(B ; R^{1} \pi_{*} \operatorname{adj} .\left(V_{5}\right)\right) \rightarrow 0,
$$

we have

$$
\begin{aligned}
& H^{0}\left(B ; R^{1} \pi_{*} \operatorname{adj} .\left(V_{5}\right)\right) \simeq H^{1}\left(Z ; \bar{U}_{4} \otimes L\right), \\
& H^{1}\left(B ; R^{0} \pi_{*} \operatorname{adj} .\left(V_{5}\right)\right) \simeq H^{1}\left(Z ; U_{4} \otimes L^{-1}\right) .
\end{aligned}
$$


Since $U_{4}$ or $\mathcal{W}_{4}$ does not have a vector bundle moduli, by construction, all the moduli of spectral cover $H^{0}\left(B ; R^{1} \pi_{*}\right.$ adj. $\left.\left(V_{5}\right)\right)$ comes from the off-diagonal block, and they are the degree of freedom called anti-generation right-handed neutrinos $\bar{N}$ in section 3.1 . They are a part of complex structure moduli in F-theory description [19]. Fibering upon this moduli are $H^{1}\left(Z ; U_{4} \otimes L^{-1}\right)$, which are now right-handed neutrinos $\bar{N}$. They are identified with the value of Ramond-Ramond 3-form field integrated over $(2,1)$ cycles of an elliptic Calabi-Yau 4 -fold of F-theory compactification. Note that the sign of $m$ and hence the constraint from the dimension-4 proton decay determines which is the base and which is the fibre. Such an identification of the origin of right-handed neutrinos in F-theory terminology will help us understand the nature of these particles better.

\subsection{F-theory Dual of $E_{8} / \mathrm{SU}(5)_{\mathrm{GUT}} \times\left\langle\mathrm{SU}(3)_{2} \times \mathrm{SU}(2)_{2} \times \mathrm{U}(1)_{\tilde{q}_{7}}\right\rangle$ and $E_{8} / \mathrm{SU}(5)_{\mathrm{GUT}} \times\left\langle\mathrm{SU}(2)_{1} \times \mathrm{SU}(2)_{2} \times \mathrm{U}(1) \times \mathrm{U}(1)\right\rangle$ Models}

Let us now turn our attention to F-theory dual description of Heterotic compactification with a $3+2$ bundle (30) or a $2+2+1$ bundle (22). The Heterotic configuration at the reducible limit can be easily mapped to the F-theory description, just as in the case of $4+1$ bundle compactification. The reducible limit can be realized only when the first Chern classes of $U(1)_{\tilde{q}_{\tau}}$ and $\mathrm{U}(1)_{\tilde{q}_{6}}$ do not have a fibre component, just like like in the $4+1$ bundle compactification with $m=0$ in (143).

The discriminant locus $\Sigma_{0}$ for the unified gauge group is of $A_{5}$ type since $\mathrm{SU}(6)_{2}$ is the maximal group that commutes with the non-Abelian part of the structure group $\mathrm{SU}(3)_{2} \times \mathrm{SU}(2)_{2}$. Likewise, the F-theory dual of $2+2+1$ bundles (22) has $D_{6}$ singular locus, since $\mathrm{SO}(12)$ $\subset E_{8}$ is the maximal group that commutes with $\mathrm{SU}(2)_{1} \times \mathrm{SU}(2)_{2}$, the non-Abelian part of the structure group.

One can also see this by following the irreducible decomposition of spectral surfaces and matter curves. Let us begin with the $3+2$ bundle compactification. Let the spectral data of vector bundles $U_{3}$ and $U_{2}$ be denoted by $\left(C_{3}, \mathcal{N}_{3}\right)$ and $\left(C_{2}, \mathcal{N}_{2}\right)$. The spectral surfaces

$$
C_{3} \sim 3 \sigma+\pi^{*} \eta_{3}, \quad C_{2} \sim 2 \sigma+\pi^{*} \eta_{2},
$$

are irreducible components of $C_{5}=C_{3}+C_{2}$. The spectral surface for the vector bundle $\wedge^{2} V_{5}$, $C_{\wedge^{2} V_{5}} \sim 10 \sigma+3 \pi^{*}\left(\eta_{3}+\eta_{2}\right)$ is split into irreducible components

$$
C_{\wedge^{2} U_{3}} \sim 3 \sigma+\pi^{*} \eta_{3}, \quad C_{U_{3} \otimes U_{2}} \sim 6 \sigma+2 \pi^{*} \eta_{3}+3 \pi^{*} \eta_{2}, \quad C_{\wedge^{2} U_{2}} \sim \sigma .
$$


Here, the first and last relations are due to (126), and one in the middle is due to ${ }^{32}$

$$
C_{V_{1} \otimes V_{2}} \sim r_{1} r_{2} \sigma+r_{1} \eta_{2}+r_{2} \eta_{1}
$$

for vector bundles $V_{i}$ with spectral covers $C_{V_{i}} \sim r_{i} \sigma+\pi^{*} \eta_{i}$.

Chiral matters in the low-energy spectrum should arise from matter curves (assuming the identification A in Table 30

$$
\begin{aligned}
\bar{c}_{\mathbf{1 0}} & =2 K_{B}+\eta_{2}=\bar{c}_{\wedge^{3} \mathbf{6}}, \\
\bar{c}_{\overline{\mathbf{5}}}=\bar{c}_{\bar{N}} & =6 K_{B}+2 \eta_{3}+3 \eta_{2}=\bar{c}_{\mathbf{6}}, \\
\bar{c}_{\bar{H}(\overline{\mathbf{5}})}\left(=\bar{c}_{\mathbf{1 0 ^ { \prime }}}\right) & =3 K_{B}+\eta_{3}=\bar{c}_{\wedge^{2} \mathbf{6}}, \\
\bar{c}_{H(\mathbf{5})} & =K_{B} \quad(\text { spread out in } B) .
\end{aligned}
$$

Various irreducible pieces of the matter curves are grouped into the matter curves of $\mathrm{SU}(6)_{2}$ multiplets in [26]. The up-type Higgs multiplet $H(5)$ is a part of $\mathrm{SU}(6)_{2}$-adj. representation and propagates over the entire base manifold $B$. The chiral 10 representations come from $\mathrm{SU}(6)_{2}-\wedge^{3} \mathbf{6}$ representation, $\overline{\mathbf{5}}$ and $\bar{N}$ from $\mathrm{SU}(6)_{2}-\overline{\mathbf{6}}$ (and $\mathbf{6}$ ) and the down-type Higgs multiplet $\bar{H}(\overline{\mathbf{5}})$ from $\mathrm{SU}(6)_{2}-\overline{\wedge^{2} \mathbf{6}}$. The Yukawa couplings arise from interactions ${ }^{33}$

$$
\begin{aligned}
& W \quad \ni \quad \wedge^{3} \mathbf{6} \cdot \mathbf{a d j} \cdot \wedge^{3} \mathbf{6}+\overline{\mathbf{6}} \cdot \wedge^{3} \mathbf{6} \cdot \overline{\wedge^{2} \mathbf{6}}+\overline{\mathbf{6}} \cdot \mathbf{a d j} \cdot \mathbf{6}, \\
& {[\rightarrow 10 \cdot H \cdot 10+\overline{\mathbf{5}} \cdot \mathbf{1 0} \cdot \bar{H}+\overline{\mathbf{5}} \cdot H \cdot \bar{N},]} \\
& {[\rightarrow \mathbf{1 0} \cdot H \cdot 10+\bar{H} \cdot \mathbf{1 0} \cdot \overline{\mathbf{5}}+\bar{H} \cdot H \cdot S \cdot]}
\end{aligned}
$$

Since the $\mathbf{1 0}$ representations and $\overline{\mathbf{5}}$ (and $\bar{N}$ ) are localised on separate matter curves, there is no reason to expect that the family structures of those representations are the same 32 . The up-type Higgs multiplet propagates in the bulk of the discriminant locus $\Sigma_{0}$. The second term, the Yukawa couplings for down-type quarks and charged leptons, involves three different matter curves, but in general, three curves on a complex 2 -fold $\Sigma_{0}$ do not necessarily meet at one point. Thus, these Yukawa couplings are generated by a membrane spanning non-locally, and may be a little small. The masses of bottom quark and tau lepton are way below the electroweak scale, and their Yukawa couplings may be small, indeed.

\footnotetext{
${ }^{32}$ Note that $\operatorname{ch}\left(V_{i}\right)=r_{i}-\sigma \cdot \pi^{*} \eta_{i}+\cdots$, and $\operatorname{ch}\left(V_{1} \otimes V_{2}\right)=\operatorname{ch}\left(V_{1}\right) \operatorname{ch}\left(V_{2}\right)=r_{1} r_{2}-\sigma \cdot\left(r_{1} \eta_{2}+r_{2} \eta_{1}\right)+\cdots$. , where Hence (150). An alternative intuitive way to understand (150) is as follows: when a spectral cover $C_{V_{i}}$ define $r_{i}$ points $\left\{p_{n_{i}}\right\}\left(n_{i}=1, \cdots, r_{i}\right)$ on a fibre $E_{b} \equiv \pi^{-1}(b)$ for $b \in B$, the spectral cover of the bundle $V_{1} \otimes V_{2}$ is given by $\left\{p_{n_{1}} \boxplus p_{n_{2}}\right\}\left(n_{1}=1, \cdots, r_{1}\right.$ and $\left.n_{2}=1, \cdots, r_{2}\right)$, where $\boxplus$ means addition under the group law of the elliptic curve $E_{b}$. The surface given by the $r_{1} r_{2}$ points in each fibre define a spectral surface given by (150).

${ }^{33}$ Note that the $\mathrm{SU}(6)_{2}$ symmetry here is a different subgroup from the $\mathrm{SU}(6)_{1}$ in (13).
} 
We have already seen that the right-handed neutrinos in the identification A in Table 3 (and singlet chiral multiplets in the identification B) are localised on a matter curve $6 K_{B}+$ $2 \eta_{3}+3 \eta_{2}$. The other way to see this is to remind ourselves that these fields describe the deformation of the vector bundle from the reducible limit (30). Using an exact sequence

$$
0 \rightarrow \mathcal{O}_{X_{1}}\left(-X_{2}\right) \rightarrow \mathcal{O}_{X} \rightarrow \mathcal{O}_{X_{2}} \rightarrow 0
$$

for $X=X_{1} \cup X_{2}$ with irreducible components $X_{1}$ and $X_{2}$, substituting $X_{1} \rightarrow C_{2}, X_{2} \rightarrow C_{3}$, and tensoring $\mathcal{O}\left(C_{5}\right)$, we see that

$$
0 \rightarrow \mathcal{O}_{C_{2}}\left(C_{2}\right) \rightarrow \mathcal{O}_{C_{5}}\left(C_{5}\right) \rightarrow \mathcal{O}_{C_{3}}\left(C_{5}\right) \rightarrow 0
$$

is also exact. Thus, the deformation of the spectral surface $C_{5}, H^{0}\left(C_{5} ; \mathcal{O}\left(C_{5}\right)\right)$, is related to the deformation of $C_{2}$ by

$$
0 \rightarrow H^{0}\left(C_{2} ; \mathcal{O}\left(C_{2}\right)\right) \rightarrow H^{0}\left(C_{5} ; \mathcal{O}\left(C_{5}\right)\right) \rightarrow H^{0}\left(C_{3} ; \mathcal{O}\left(C_{5}\right)\right)
$$

$H^{0}\left(C_{3} ; \mathcal{O}\left(C_{5}\right)\right)$ is further related to the deformation of the spectral surface $C_{3}$ by another exact sequence

$$
0 \rightarrow \mathcal{O}_{C_{3}}\left(C_{5}-C_{2}\right) \rightarrow \mathcal{O}_{C_{3}}\left(C_{5}\right) \rightarrow \mathcal{O}_{C_{3} \cdot C_{2}}\left(C_{5}\right)
$$

It follows that

$$
0 \rightarrow H^{0}\left(C_{3} ; \mathcal{O}\left(C_{3}\right)\right) \rightarrow H^{0}\left(C_{3} ; \mathcal{O}\left(C_{5}\right)\right) \rightarrow H^{0}\left(C_{3} \cdot C_{2} ; \mathcal{O}\left(C_{5}\right)\right)
$$

is also exact. Thus, the deformation of the spectral surface $C_{5}$ is decomposed into three pieces. Two of them are the deformation of the spectral surfaces of the bundle $U_{2}$ and $U_{3}$, $H^{0}\left(C_{2} ; N_{C_{2} \mid Z}\right)$ and $H^{0}\left(C_{3} ; N_{C_{3} \mid Z}\right)$. The other one $H^{0}\left(C_{2} \cdot C_{3} ; \mathcal{O}\left(C_{5}\right)\right)$ describes the deviation from the reducible limit and is localised at the intersection of $C_{2} \cdot C_{3}$. Its projection on $B$ is $6 K_{B}+2 \eta_{3}+3 \eta_{2}$, which is the same as $\bar{c}_{\bar{N}}$ determined above.

In the $2+2+1$ bundle compactification of the Heterotic theory, the spectral surface $C_{3} \sim$ $3 \sigma+\pi^{*} \eta_{3}$ further splits into irreducible pieces $\sigma$ and $2 \sigma+\pi^{*} \eta_{3}$. The matter curves rearrange themselves accordingly, and become

$$
\begin{aligned}
\bar{c}_{\mathbf{1 0}}=\bar{c}_{\overline{\mathbf{5}}}=\bar{c}_{\bar{N}}= & 2 K_{B}+\eta_{2}=\bar{c}_{\mathbf{3 2}^{\prime}}, \\
\text { (absent in LE.) } & 2\left(2 K_{B}+\eta_{3}+\eta_{2}\right)=\bar{c}_{\text {vec. }}, \\
\text { (absent in LE.) } & 2 K_{B}+\eta_{3}=\bar{c}_{\mathbf{3 2}}, \\
\bar{c}_{H(\mathbf{5})}=\bar{c}_{\bar{H}(\overline{\mathbf{5}})}= & K_{B} \quad(\text { spread out in } B) .
\end{aligned}
$$


Here, both the $H(\mathbf{5})$ and $\bar{H}(\overline{\mathbf{5}})$ multiplets arise within the $\mathrm{SO}(12)$ gauge group, and their wavefunctions are not confined on a matter curve.

The SU(6) gauge symmetry in the F-theory dual of the $3+2$ bundle compactification or $\mathrm{SO}(12)$ gauge symmetry dual to the $2+2+1$ bundle compactification are broken by the $\mathrm{U}(1)$ bundles on the discriminant locus $\Sigma_{0}$. If the Fayet-Iliopoulos parameters of those $\mathrm{U}(1)$ symmetries are non-zero, some fields charged under the symmetry develop expectation values, and this correspond to the extension structure of vector bundles in (91) and (92) in Heterotic theory vacua. The same constraint on the sign of the Fayet-Iliopoulos parameters as in section 3 has to be imposed here.

A story similar to section 5.2.3 can be told about the F-theory dual of $3+2$ bundle and $2+2+1$ bundle compactification of Heterotic theory, but we just do not do it here.

\subsection{Toward a Description Intrinsic to F-theory}

\subsubsection{Characterising Matter Curves within F-theory}

We have so far provided some examples of F-theory vacua that do not have dimension-4 proton decay operators. All of them are dual to certain Heterotic vacua, since they were obtained through the Heterotic-F-theory duality. But not all the F-theory vacua are dual to Heterotic vacua. If we had only handles on vacua that are dual to Heterotic theory, we would lose a chance to study phenomenology of F-theory vacua that do not have Heterotic dual. Thus, at the end of this section, we take a little step to try to phrase some aspects of F-theory vacua in a way intrinsic to F-theory. That will help further explore F-theory vacua that are phenomenologically viable.

Matter curves, or loci of enhanced singularity, have been discussed, but they were described by divisors $\eta$ on the base manifold $B$ and its definition was associated with the spectral cover (or the second Chern class) of vector bundles in Heterotic compactification. It is rather unsatisfactory that the matter curves, which are generic features of F-theory vacua, are described in language intrinsic to Heterotic theory. It would be nice if the matter curves are characterized without referring to such objects in Heterotic theory as vector bundles.

In the case of $4+1$ model at the reducible limit, the line bundles $L$ and $\operatorname{det} U_{4}$ add twists only on the matter curves and they do not change the matter curves themselves. The discriminant locus $\Sigma_{0} \simeq B$ has $D_{5}$ singularity, supporting $\mathrm{SO}(10)$ gauge symmetry if seen locally. The matter curves are those of theories with unbroken $\mathrm{SO}(10)$ symmetry. We do not question

if this result found in the Heterotic-F-theory dual region holds true in generic F-theory vacua 
without dimension-4 proton decay. But we would like to ask the following question: how we define $\eta$ to begin with, and whether there is an extra freedom in choosing matter curves, when an elliptic fibration $\pi_{X}: X \rightarrow B^{\prime}$ of an F-theory vacuum is not of a $K 3$-fibration. The following content is closely related to what was discussed in 67.

Let us consider an F-theory compactification on an elliptic fibred $\mathrm{CY}_{4} \pi: X \rightarrow B^{\prime}$ given by

$$
y^{2}=x^{3}+f\left(z_{1}, z_{2}, z_{3}\right) x+g\left(z_{1}, z_{2}, z_{3}\right),
$$

where $\left(z_{1}, z_{2}, z_{3}\right)$ are local coordinates of the base manifold $B^{\prime},(y, x)$ are those of the elliptic fibre, and $(x, y, f, g)$ are sections of $\mathcal{O}\left(-2 K_{B^{\prime}}\right), \mathcal{O}\left(-3 K_{B^{\prime}}\right), \mathcal{O}\left(-4 K_{B^{\prime}}\right)$, and $\mathcal{O}\left(-6 K_{B^{\prime}}\right)$, respectively. The determinant of this elliptic curve $\Delta \equiv 4 f^{3}+27 g^{2}$ is a section of $\mathcal{O}\left(-12 K_{B^{\prime}}\right)$, whose zero locus determines the discriminant locus on the base manifold $B^{\prime}$. Thus, the following consistency condition has to be satisfied [17]

$$
\operatorname{div} \Delta=-12 K_{B^{\prime}}
$$

This condition reduces to the Ramond-Ramond charge cancellation condition of 7-branes in the Type IIB limit.

Now let us assume that one of irreducible components of the discriminant locus is of $D_{5}$ type singularity, supporting $\mathrm{SO}(10)$ unbroken gauge symmetry. Let us denote the irreducible component by $\Sigma$, i.e.,

$$
\operatorname{div} \Delta=7 \Sigma+\cdots
$$

At the loci where other irreducible components intersect $\Sigma$, singularity and gauge symmetry are enhanced, and matter is localised. The consistency condition (168) has to be satisfied in any F-theory compactification, and in particular, when evaluated on any divisors of the base manifold $B^{\prime}$. Assuming that there are only matters ${ }^{34}$ in the $\mathrm{SO}(10)-\mathbf{1 6}$ and -vec., the condition (168) becomes

$$
7 \Sigma \cdot \Sigma+3 \bar{c}_{16}+\bar{c}_{\text {vec. }}=-12 K_{B^{\prime}} \cdot \Sigma
$$

when evaluated on $\Sigma$. The integer coefficients in front of matter curves are read out from the behaviour of the determinant around the matter curve found in [26]. Let us now define

\footnotetext{
${ }^{34} \mathrm{We}$ restrict ourselves to this limited matter contents because we are interested in F-theory geometry relevant to the real world in this article; there is no strong phenomenology motivation (except in an application to some models of Dimopoulos-Wilczek mechanism) to think of geometry that yields multiplets in the symmetric tensor representation.
} 
a divisor $\eta$ on the subvariety $\Sigma$. Since $\Sigma \subset B^{\prime}$ defines a divisor on $\Sigma$ itself through the self-intersection, let us define a divisor $\eta$ on $\Sigma$ through [57]

$$
\eta \equiv \Sigma \cdot \Sigma-6 K_{\Sigma}
$$

This is a generalization of (130). Using the adjunction formula

$$
K_{\Sigma}=\left.K_{B^{\prime}}\right|_{\Sigma}+\Sigma
$$

we see that the general solution to (170) is of the form

$$
\bar{c}_{\mathbf{1 6}}=\eta+4 K_{\Sigma}+\eta^{\prime}, \quad \text { and } \quad \bar{c}_{\mathbf{v e c} .}=2 \eta+6 K_{\Sigma}-3 \eta^{\prime}
$$

where $\eta^{\prime}$ is an arbitrary divisor on $\Sigma$.

Another theoretical constraint is the box anomaly cancellation. On an arbitrary curve in $\Sigma$, the budget of the inflow of irreducible box anomalies should balance. It is equivalent to the Ramond-Ramond charge cancellation in Type IIB string theory, but it seems it is not equivalent to (168) in F-theory in general. Since the $[\mathrm{SO}(10)]^{4}$ irreducible anomaly comes from -2 units from hypermultiplets in $\mathbf{1 6}$ representation and +1 units from half-hypermultiplets in vec representation, the arbitrariness $\eta^{\prime}$ in the above general solutions changes the total amount of the irreducible box anomaly. Thus, the anomaly cancellation condition eliminates the arbitrariness, and since we know that $\eta^{\prime}=0$ is a consistent solution, that should be the only solution. This implies that the contribution to the irreducible box anomaly from $\mathrm{SO}(10)$-adj. representation, including both the contribution from the vector multiplet and hypermultiplets, is given by $K_{\Sigma}$ so that

$$
2 \bar{c}_{\text {adj. }}-2 \bar{c}_{\mathbf{1 6}}+\bar{c}_{\text {vec. }}=2 K_{\Sigma}-2\left(\eta+4 K_{\Sigma}\right)+\left(2 \eta+6 K_{\Sigma}\right)=0 .
$$

Therefore, the matter curves given in (127) and (128) are of general form in F-theory vacua, valid even when there is not Heterotic dual. The divisor $\eta$ is now defined only from local information of the geometry of F-theory through (171), and the matter curves are determined by this divisor.

Exactly the same argument can be developed for the matter curves on a discriminant locus $\Sigma$ with SU(6) or SO(12) gauge symmetry on it. This is a situation relevant to the reducible limit of the $3+2$ and $2+2+1$ bundle compactification of Heterotic theory. The divisor $\eta$ on $\Sigma$ is defined by (171). There are three different matter curves to think about, namely $\bar{c}_{\wedge^{3} \mathbf{6}}$, 
$\bar{c}_{\mathbf{6}}$ and $\bar{c}_{\wedge^{2} \mathbf{6}}$ for $\mathrm{SU}(6)$, and $\bar{c}_{\mathbf{3 2}^{\prime}}, \bar{c}_{\mathbf{v e c}}$ and $\bar{c}_{\mathbf{3 2}}$ for $\mathrm{SO}(12)$. On the other hand, there are two independent constraints, (168) and the irreducible box anomaly cancellation:

$$
\begin{aligned}
6 \Sigma \cdot \Sigma+3 \bar{c}_{\wedge^{3} \mathbf{6}}+\bar{c}_{\mathbf{6}}+4 \bar{c}_{\wedge^{2} \mathbf{6}}=-12 K_{B^{\prime}} \cdot \Sigma, \\
6 K_{\Sigma}-3 \bar{c}_{\wedge^{3} \mathbf{6}}+\bar{c}_{\mathbf{6}}-2 \bar{c}_{\wedge^{2} \mathbf{6}}=0
\end{aligned}
$$

for $\mathrm{SU}(6)$ and

$$
\begin{aligned}
8 \Sigma \cdot \Sigma+2 \bar{c}_{\mathbf{3 2}^{\prime}}+\bar{c}_{\text {vec. }}+2 \bar{c}_{\mathbf{3 2}} & =-12 K_{B^{\prime}} \cdot \Sigma \\
4 K_{\Sigma}-2 \bar{c}_{\mathbf{3 2}^{\prime}}+\bar{c}_{\text {vec. }}-2 \bar{c}_{\mathbf{3 2}} & =0
\end{aligned}
$$

for $\mathrm{SO}(12)$. Thus, general solutions have one degree of freedom left in choosing divisors. They are given by (151)-(154) and (163)-(166), respectively, with the freedom of how to split the divisor $\eta$ defined in (171) into $\eta_{2}$ and $\eta_{3}$. Thus, even in generic F-theory vacua that may not have Heterotic dual, the matter curves are constrained just as in vacua with Heterotic dual.

\subsubsection{Chirality Formula}

There is another expression that allows straightforward generalization to F-theory vacua. Let us look at the expression for the chirality for the $\mathbf{1 0}^{\prime}$ representation in (129), in a way presented in section 5.2.2 Although it arises from a part of the $\mathrm{SO}(10)$ gauge field of the discriminant locus $\Sigma_{0} \sim B$, and is not localised on a specific matter curve, $\sigma \cdot C_{1} \sim \sigma \cdot K_{B}$ can be formally used as its matter curve. The line bundle $\mathcal{N}_{1}$ is $L$ itself. Applying (136) and (135) formally, along with (141), we obtain ${ }^{35}$

$$
\# \mathbf{1 0}-\# \overline{\mathbf{1 0}}^{\prime}=\int_{K_{\Sigma_{0}}} c_{1}(L)
$$

This formula is a generalisation of the corresponding expression in the Type IIB string theory. Indeed, let us think of a Type IIB configuration where five D7-branes are wrapped on a holomorphic 4-cycle $\Sigma$ along with an O7-plane and five D7-branes as the orientifold mirror image of the original D7-branes. Those branes support $\mathrm{SO}(10)$ gauge group. When a U(1) flux $F$ is turned on on the five D7-branes (and on their images) so that SU(5) gauge group is left unbroken, we have

$$
\# \mathbf{1 0}^{\prime}-\# \overline{\mathbf{1 0}}^{\prime}=2 \int_{\Sigma \cdot \Sigma=K_{\Sigma}}\left(\frac{F}{2 \pi}-\frac{B}{(2 \pi)^{2} \alpha^{\prime}}\right)=-\int_{\Sigma} c_{1}(T \Sigma) 2\left(\frac{F}{2 \pi}-\frac{B}{(2 \pi)^{2} \alpha^{\prime}}\right) .
$$

\footnotetext{
${ }^{35}$ The phenomenological constraint (42) becomes $K_{B} \cdot c_{1}(L)=0$ in elliptic Calabi-Yau compactification of the Heterotic theory. Thus (179) becomes zero. This fact itself is nothing more than a straightforward consequence of the phenomenological requirement (41).
} 
The difference in the factor 2 is due to the normalisation of $U(1)$ charges: the $\mathbf{1 0}^{\prime}$ representations in (179) have $q_{L}=1$ unit of charge under the line bundle $L$ on the 4-cycle $\Sigma_{0} \sim B$, whereas the normalisation of $F$ in (180) is set so that the $\mathbf{1 0}^{\prime}$-representations have 2 units of charge - one unit from each end of open strings connecting D7-branes on the both sides of an O7-plane. We have ignored the B-field. Thus, (179) suggests that the expression in Type IIB string theory (180) is generalized straightforwardly in the F-theory as in (179). It should be kept in mind that we should use $K_{\Sigma}$, rather than $\Sigma \cdot \Sigma$, in the F-theory version. They are not the same in the F-theory, since the half of Calabi-Yau 3-folds for the Type IIB orientifold compactifications are projected out to be $B^{\prime}$, which is not a Calabi-Yau manifold.

The chirality formula (179) has an immediate application. One can ask a following question: certainly it is a generic feature of F-theory vacua that matter multiplets are localised on codimension-1 subspace, matter curves, in a discriminant locus; but is it possible to have a vacuum where all chiral matters are not localised but propagate over the entire "bulk" $\Sigma$ ? In other words, is it possible to think of a vacuum where all matters arise from a stack of parallel 7-branes with $E_{7}$ or $E_{8}$ gauge group on it, rather than from a system of intersecting 7-branes? Reference 68] contains examples of F-theory geometry of parallel 7-branes supporting $E_{7}$ and $E_{8}$. When a vector bundle is turned on as in the discussion in section 2 and 3. various low-energy particles are obtained with the right Yukawa couplings. Thus, one can think of such a vacuum theoretically. But we see by using the chirality formula above, that parallel 7-branes with $E_{8}$ gauge symmetry cannot yield a net chirality in the low-energy spectrum.

It is important in (179) that a) only the first Chern class matters and b) only the first Chern classes on one curve $K_{\Sigma}$ in $S$ are relevant. In all the three symmetry breaking patterns in section 2 with the underlying $E_{8}$ symmetry, multiplets in the $\mathrm{SU}(5)_{\text {GUT }} \mathbf{1 0}$ representation arise from a rank-5 bundle with the structure group in $\mathrm{SU}(5)$. Thus, the overall net chirality is given by the sum of $\int_{K_{\Sigma}} c_{1}$ of each irreducible bundle, which vanishes because $\operatorname{det} V_{5} \simeq \mathcal{O}_{\Sigma}$. Thus, we cannot think of a vacuum where all the matter curves of $\mathrm{SU}(5)_{\mathrm{GUT}} \mathbf{- 1 0}$ representation are $K_{\Sigma}$ in order to have a net chirality in the low-energy spectrum. Some multiplets in the $\mathrm{SU}(5)_{\mathrm{GUT}} \mathbf{- 1 0}$ representation have to be localized on $\Sigma$. This also means that the isolated discriminant locus with $E_{8}$ gauge symmetry does not lead to a realistic vacuum.

The matter curves and the gauge symmetries on the discriminant locus $\Sigma$ are relevant to particle physics, primarily through the determination of Yukawa couplings. Although they are important, we have little chance to see them directly, as long as the compactification radius remains very small compared with the length scale of the electroweak scale. But there is a 
situation where the gauge symmetry in the bulk is of more importance: the gauge mediation of supersymmetry breaking. The gravitino problem of supersymmetric theories suggests that either the gravitino mass is very heavy or very light $\left(m_{3 / 2} \lesssim 10 \mathrm{eV}\right)$. Supersymmetry breaking should be mediated through gauge interactions in the latter scenario, and there is a phenomenology model that naturally realizes gauge mediation with very low gravitino mass [69]. It may be realized in Type IIB string / F-theory with a resolved conifold geometry with warped factor [73] where D7-branes for the $\mathrm{SU}(5)_{\text {GUT }}$ gauge group are wrapped on $S^{3}$. In such models, low-lying Kaluza-Klein states of the bulk vector multiplets are observable in LHC experiments.

Thus, the gauge symmetry, $\mathrm{SO}(10), \mathrm{SU}(6), \mathrm{SO}(12)$ or just $\mathrm{SU}(5)$, makes an observable difference. The above argument suggests, however, that it is unlikely to see the entire $E_{8}$ gauge group. There are also lots of phenomenological model buildings on the origin of fermion mass hierarchies on AdS background, and the determination of the matter curves provides restrictions on the degree of freedom available in such effective-field theory model buildings.

\section{Summary and Discussion}

Gauge theories are often accompanied with 16 supersymmetry charges at the microscopic level, and when a large gauge symmetry $G$ is spontaneously broken down to a smaller group $H$, charged matter multiplets arise from the coset space of $\mathfrak{g} / \mathfrak{h}$. This is a salient feature of string theory, and this is a framework where we can expect to unify interactions of quark, lepton, neutrino and Higgs fields with gauge bosons.

\section{Physics Summary}

Requiring that the tri-linear Yukawa interactions be generated from the super Yang-Mills interactions, while the dimension- 4 proton decay operators be not generated, we saw that $G=E_{7}$ is the minimal choice and $G=E_{8}$ is the only alternative, when we impose GeorgiGlashow SU(5) unification of quarks and leptons. There are four different patterns of how to break these symmetries to $\mathrm{SU}(5)_{\mathrm{GUT}}$, namely,

$$
\begin{array}{ll}
E_{8} / \mathrm{SU}(5)_{\mathrm{GUT}} \times\left\langle\mathrm{SU}(4) \times \mathrm{U}(1)_{\chi}\right\rangle & \mathrm{A}, \\
E_{8} / \mathrm{SU}(5)_{\mathrm{GUT}} \times\left\langle\mathrm{SU}(3)_{2} \times \mathrm{SU}(2)_{2} \times \mathrm{U}(1)_{\tilde{q}_{7}}\right\rangle & \mathrm{A}, \mathrm{B}, \\
E_{8} / \mathrm{SU}(5)_{\mathrm{GUT}} \times\left\langle\mathrm{SU}(2)_{1} \times \mathrm{SU}(2)_{2} \times \mathrm{U}(1) \times \mathrm{U}(1)\right\rangle & \mathrm{A}, \mathrm{B}, \\
E_{7} / \mathrm{SU}(5)_{\mathrm{GUT}} \times\left\langle\mathrm{SU}(2)_{2} \times \mathrm{U}(1) \times \mathrm{U}(1)\right\rangle & \mathrm{A}, \mathrm{B} .
\end{array}
$$

We might call them, 41A-, 32A(B)-, 221A(B)- and 21A(B)-scenarios, for brevity. The origins of all the low-energy multiplets are identified in the coset spaces, and labels $\mathrm{A}$ and $\mathrm{B}$ 
correspond to different particle identifications. A is when moduli multiplets are identified with right-handed neutrinos, and in case B, they are identified with a singlet chiral field that has $W \ni S H \bar{H}$ coupling. In all these possibilities, all the Yukawa couplings for the quarks and charged leptons are generated from the super Yang-Mills interactions, and in possibilities A, Dirac Yukawa couplings of neutrinos also exist. We have not discussed how to break SU(5) GUT symmetry in this article. All U(1) vector fields that appear above have Green-Schwarz couplings, and are massive (68). Thus, they are not likely to be within the range of terrestrial experiments.

The dimension-4 proton decay operators are forbidden by U(1) global symmetries that correspond to the anomalous U(1) gauge symmetries. When the Fayet-Iliopoulos parameters does not vanish, the U(1) symmetries can be spontaneously broken by fields of the same sign charges. When the sign of the Fayet-Iliopoulos parameter and the charges are appropriate, dimension-4 operators are not generated in any perturbative processes. In all four A-scenarios, either right-handed neutrinos or their anti-generation particles develop expectation values to absorb the Fayet-Iliopoulos parameters, and hence $\mathbb{Z}_{2} B-L$ parity is not preserved anymore, yet the absence of dimension-4 proton decay is guaranteed.

All possible terms in superpotential can be worked out, imposing all the underlying symmetry, and allowing to insert spurions that spontaneously break them. Using this approach, effective operators of some of the scenarios were studied in this article. See (172), (73), (90), (93) and (94) and discussion around these equations. All the discussion so far are very robust, and does not depend on whether a vacuum is realized in Heterotic theory, M-theory or F-theory.

We also argued, in the context of M-theory compactification, that diagonal entries of up-type quark Yukawa matrix tend to be suppressed in some part of the moduli space.

In F-theory vacua, $\mathrm{SU}(5)_{\text {GUT }}$ gauge field propagates on an internal 4-cycle, and the zeromode wave functions are localized on internal 2-cycles, in general. When the Fayet-Iliopoulos parameters vanish, we determined how the low-energy particles are localized, and found that the up-type Higgs multiplet propagates on the whole 4-cycle and gauge symmetry is locally $\mathrm{SU}(6)$ in 32A,B scenarios, both up-type and down-type Higgs multiplets propagate on the 4-cycle and the gauge symmetry is locally $\mathrm{SO}(12)$ in the $221 \mathrm{~A}$ scenario. If the 4-cycle of the $\mathrm{SU}(5)_{\mathrm{GUT}}$ is extended to a warped extra dimension explaining the hierarchy between the Planck scale and the electroweak scale, we can see at LHC the Kaluza-Klein vector fields of such an enhanced gauge symmetry.

\section{String Theory Discussion}


The number of consistent vacua in String Theory continues to grow, while there is no prospect in near future that some non-perturbative dynamics is found and the overdegeneracy of vacua is lifted (see [74] for a full list of references). At least we may have to live with the multitude of vacua for a while, and there have been some attempts at finding constraints or predictions that hold for wide class of string vacua [75, 76]. There is another kind approach, where people try to find out dynamics that takes place over the landscape of vacua [77] and to find out unknown "structure" the true landscape has [7].

We found that the supersymmetric SU(5) landscape contains seven islands. Islands may be connected by continuous deformation of moduli parameters, but proton decays too fast in vacua between the islands, and "lives" may not exist in such vacua. The minimal underlying symmetry of SU(5) unified theory is $E_{7}$, and D-brane Yang-Mills interactions in Type I, IIA or IIB string theory cannot provide the form of the up-type quark Yukawa couplings of SU(5) unified theories.

The framework presented in this article does not have a massless $\mathrm{U}(1)_{B-L}$ gauge boson, and yet, it is now clear when dimension-4 proton decay operators are absent. In Heterotic language, a rank-5 holomorphic stable vector bundle $V_{5}$ has to have a certain sub-bundle, in order to prevent the dimension- 4 proton decay. The condition will be easily taken into account in search for a geometry describing our world. This condition has also been translated into M-theory and F-theory compactification, partially but not completely yet.

The Yukawa couplings of quarks and leptons come from super Yang-Mills interactions. The origin of low-energy particles has been identified in Heterotic, M and F-theory language. Thus, one can exploit various techniques in string theory to extract more information.

\section{A Note on Lie Algebra}

\section{A.1 $\mathfrak{e}_{6}$ Lie algebra}

The $\mathfrak{e}_{6}$ Lie algebra contains $\mathfrak{s u}(5)+\mathfrak{s u}(2)_{2}+\mathfrak{u}(1)$ subalgebra. After removing the nodes for $\alpha_{2}$ and $-\theta$ from the extended Dynkin diagram of $E_{6}$ (see Fig. 1), one node $\alpha_{1}$ for $\mathfrak{s u}(2)_{2}$ and four $\alpha_{3,4,5,6}$ for $\mathfrak{s u}(5)$ are left. The irreducible decomposition of the $\mathfrak{e}_{6}$-adj. representation under the $\mathfrak{s u}(5)+\mathfrak{s u}(2)_{2}+\mathfrak{u}(1)$ subalgebra (4) is known in the literature. We need to know, however, which roots of $\mathfrak{e}_{6}$ correspond to which irreducible components in order to provide full geometric description in M-theory, the equation (44) is not enough. We first follow an intuitively tractable way manipulating the extended Dynkin diagram to roughly determine 
the roots of the irreducible components and later provide the full results of explicit calculation.

When only the node of $\alpha_{2}$ is removed from the extended Dynkin diagram of $E_{6}$, the $\mathfrak{e}_{6}$-adj. representation is decomposed into

$$
\operatorname{Res}_{\mathrm{SU}(6) \times \mathrm{SU}(2)_{2}}^{E_{6}}\left(\mathfrak{e}_{6} \text {-adj. }\right) \simeq(\mathbf{a d j} ., \mathbf{1}) \oplus(\mathbf{1}, \mathbf{a d j} .) \oplus\left(\wedge^{3} \mathbf{6}, \mathbf{2}\right) .
$$

Thus the roots in the $\left(\wedge^{3} \mathbf{6}, \mathbf{2}\right)$ representation contain $\alpha_{2}$. Since this representation is a doublet of $\mathrm{SU}(2)_{2}$ generated by $\alpha_{1}$, half of roots in the representation contain the image of $\alpha_{2}$ under the Weyl reflection associated with $\alpha_{1}$, i.e., $\alpha_{1}+\alpha_{2}$.

When the node of $-\theta$ is further removed from the $A_{5}$ Dynkin diagram made of $\alpha_{3,4,5,6}$ and $-\theta$, breaking the $\mathrm{SU}(6)$ symmetry down to $\mathrm{SU}(5) \times \mathrm{U}(1)$, the $\left(\wedge^{3} \mathbf{6}, \mathbf{2}\right)$ representation splits up into $(\mathbf{1 0}, \mathbf{2})^{1}+$ h.c. under the $\mathrm{SU}(5) \times \mathrm{SU}(2)_{2} \times \mathrm{U}(1)$, and $\mathrm{SU}(6)$-adj. representation into SU(5)-adj., $-5^{-2}+$ h.c. and a singlet. Thus, we have the irreducible decomposition (4). Roots in the SU(5)-5 representation (and its Hermitian conjugate) contain $-\theta$.

One can also carry out an explicit calculation, using a basis in the dual space of Cartan subalgebra. We adopt the basis in [79] for $\mathfrak{e}_{d}(d=6,7,8)$ algebras, which can treat all three exceptional Lie algebra systematically and has also clear interpretation in del Pezzo surfaces. When the simple roots are chosen as

$$
\begin{aligned}
\alpha_{i} & =L_{i}-L_{i+1} \text { for } i=1, \cdots,(d-1), \quad \text { and } \\
\alpha_{d} & =L_{0}-\left(L_{1}+L_{2}+L_{3}\right)
\end{aligned}
$$

with $d=6$, all of positive roots are

$$
\begin{aligned}
& L_{i}-L_{j} \quad(i<j), \\
& L_{0}-\left(L_{i}+L_{j}+L_{k}\right) \quad(i<j<k) \quad \text { and } \\
& \theta \equiv 2 L_{0}-\left(L_{1}+\cdots+L_{6}\right)=\alpha_{1}+2 \alpha_{2}+3 \alpha_{3}+2 \alpha_{4}+\alpha_{5}+2 \alpha_{6},
\end{aligned}
$$

where $\theta$ is the highest root. Under this convention, each irreducible component consists of roots shown as follows:

- (1,adj.): $\pm \alpha_{1}= \pm\left(L_{1}-L_{2}\right)$.

- (adj.,1): $\pm\left(L_{i}-L_{j}\right)(3 \leq i<j \leq 6)$ and $\pm\left(L_{0}-\left(L_{1}+L_{2}+L_{k}\right)\right)(k=3, \cdots, 6)$.

- $\overline{\left(\wedge^{2} 5,2\right)}$ :

$L_{0}-\left(L_{1}+L_{2}+L_{i}+L_{j}\right)+L_{a} \quad(a=1,2$ and $3 \leq i<j \leq 6)$ and $L_{a}-L_{k}(a=1,2$ and $k=3, \cdots, 6)$.

The lowest weights are a doublet $\left(\alpha_{1}+\alpha_{2}, \alpha_{2}\right)=\left(L_{a}-L_{3}\right)_{a=1,2}$. 
- $\left(\wedge^{4} \overline{5}, \mathbf{1}\right) \simeq(\mathbf{5}, \mathbf{1})$ :

$L_{0}-\left(L_{i}+L_{j}+L_{k}\right)(3 \leq i<j<k \leq 6)$ and

$\theta=2 L_{0}-\left(L_{1}+\cdots+L_{6}\right)$.

The highest weight is $\theta=\left(\alpha_{1}+2 \alpha_{2}\right)+\left(3 \alpha_{3}+2 \alpha_{4}+\alpha_{5}+2 \alpha_{6}\right)$.

The roots of $\left(\wedge^{2} \mathbf{5}, \mathbf{2}\right)$ and $(\overline{\mathbf{5}}, \mathbf{1})$ representations of $\mathrm{SU}(5) \times \mathrm{SU}(2)_{2}$ are obtained by just multiplying $(-1)$ to those of their Hermitian conjugate representations. In M-theory description, M2-branes wrapped on $\left\{-C_{-\theta},-\left(C_{-\theta}+C_{6}\right),-\left(C_{-\theta}+C_{6}+C_{3}\right),-\left(C_{-\theta}+C_{6}+C_{3}+C_{4}\right),-\left(C_{-\theta}+\right.\right.$ $\left.\left.C_{6}+C_{3}+C_{4}+C_{5}\right)\right\}$ provide the quintet $H(5)$ particles, where $C_{i}$ 's are 2-cycles corresponding to the simple roots $\alpha_{i}$ 's.

\section{A.2 $\mathfrak{e}_{7}$ Lie algebra}

The $\mathfrak{e}_{7}$ Lie algebra contains an $\mathfrak{s u}(6)_{1}+\mathfrak{s u}_{2}+\mathfrak{u}(1)$ subalgebra, and its subalgebra $\mathfrak{s u}(5)+$ $\mathfrak{s u}_{2}+\mathfrak{u}(1)+\mathfrak{u}(1)$. They are obtained by removing the nodes of $\alpha_{2}$, and $-\theta$ (and $\left.\alpha_{7}\right) .{ }^{36}$ The $\mathfrak{s u}(2)_{2}$ is generated by $\alpha_{1}, \mathfrak{s u}(6)_{1}$ by $\alpha_{7,3,4,5,6}$, and $\mathfrak{s u}(5)$ by $\alpha_{3,4,5,6}$ (see Fig. 2 ).

When only the node of $\alpha_{2}$ is removed from the extended Dynkin diagram Fig. 2, $E_{7}$ is reduced to $\mathrm{SU}(3) \times \mathrm{SU}(6)_{1}$, and

$$
\operatorname{Res}_{\mathrm{SU}(6)_{1} \times \mathrm{SU}(3)_{1}}^{E_{7}}(\mathfrak{e}-\mathbf{a d j} .) \simeq(\mathbf{a d j} ., \mathbf{1}) \oplus(\mathbf{1}, \mathbf{a d j} .) \oplus\left(\wedge^{2} \mathbf{6}, \overline{\mathbf{3}}\right) \oplus \text { h.c.. }
$$

All the roots in the $\left(\wedge^{2} \mathbf{6}, \overline{\mathbf{3}}\right)+$ h.c. representation (and its Hermitian conjugate) should contain $\alpha_{2}$, and form a triplet $\pm\left(-\theta+\alpha_{1}+\alpha_{2}, \alpha_{1}+\alpha_{2}, \alpha_{2}\right)$ under the $\mathrm{SU}(3)_{1}$ symmetry; linear combinations of $\alpha_{3, \cdots, 7}$ are just omitted here. Upon further removing the node of $-\theta$, the $\mathrm{SU}(3)_{1}$ symmetry is reduced to $\mathrm{SU}(2)_{2} \times \mathrm{U}(1)_{q_{6}}$, and each component splits into irreducible pieces;

$$
\begin{aligned}
\left(\wedge^{2} \mathbf{6}, \overline{\mathbf{3}}\right) & \rightarrow\left(\wedge^{2} \mathbf{6}, \mathbf{2}\right)^{1} \oplus\left(\wedge^{2} \mathbf{6}, \mathbf{1}\right)^{-2} \\
(\mathbf{1}, \mathbf{a d j} .) & \rightarrow(\mathbf{1}, \mathbf{a d j} .)^{0} \oplus(\mathbf{1}, \mathbf{1})^{0} \oplus(\mathbf{1}, \mathbf{2})^{3} \oplus \text { h.c. }
\end{aligned}
$$

Thus, the irreducible decomposition (9) is obtained. Roots in the representation $(\mathbf{1}, \mathbf{2})^{3}+$ h.c. should be a doublet $\pm\left(-\theta,-\theta+\alpha_{1}\right)$. The roots in $\left(\wedge^{2} \mathbf{6}, \mathbf{1}\right)^{-2}+$ h.c. are $\pm\left(-\theta+\alpha_{1}+\alpha_{2}\right)$, and those in $\left(\wedge^{2} \mathbf{6}, \mathbf{2}\right)^{1}+$ h.c. are a doublet $\pm\left(\alpha_{1}+\alpha_{2}, \alpha_{2}\right)$, up to some linear combinations of $\alpha_{3, \cdots, 7}$

\footnotetext{
${ }^{36} \mathrm{It}$ is also fine to remove $\alpha_{1}$ instead of $-\theta$. The node for $\alpha_{1}$ is identified with the root of $\mathfrak{s u}(2)_{2}$ subalgebra only because we want to use the same $\mathfrak{s u}(2)_{2}$ in the $\mathfrak{e}_{8}$ algebra in section A.3.
} 
When the node of $\alpha_{7}$ is further removed, the symmetry is reduced from $\mathrm{SU}(6)_{1}$ to $\mathrm{SU}(5)$, and $\mathrm{SU}(6)$-charged representations split into smaller irreducible pieces. In order to determine whether the roots of $\mathrm{SU}(5) \times \mathrm{SU}(2)_{2}$ irreducible representations contain $\alpha_{7}$ or not, let us consider another route of the irreducible decomposition. We could have remove $\alpha_{7}$ first, and $\alpha_{2}$ next, and $-\theta$ at the end; the symmetry is broken through $E_{7}, \mathrm{SU}(8), \mathrm{SU}(5) \times \mathrm{SU}(3)_{1} \times \mathrm{U}(1)$, and finally to $\mathrm{SU}(5) \times \mathrm{SU}(2)_{2} \times \mathrm{U}(1) \times \mathrm{U}(1)$. In the first step in the new route,

$$
\operatorname{Res}_{\mathrm{SU}(8)}^{E_{7}}\left(\mathfrak{e}_{7} \text {-adj. }\right) \simeq \operatorname{adj} . \oplus \wedge^{4} \mathbf{8}
$$

and $\wedge^{4} \mathbf{8}$ representation further reduces to $[(\mathbf{1}, \mathbf{5}) \oplus(\mathbf{3}, \overline{\mathbf{1 0}})] \oplus$ h.c. when $\mathrm{SU}(8)$ is reduced to $\mathrm{SU}(5) \times \mathrm{SU}(3)_{1} \times \mathrm{U}(1)$. Thus, among the irreducible components $(\mathbf{1 0}, \overline{\mathbf{3}}) \oplus(\mathbf{5}, \overline{\mathbf{3}})=\left(\wedge^{2} \mathbf{6}, \overline{\mathbf{3}}\right)$, the former comes from $\mathrm{SU}(8)-\wedge^{4} \boldsymbol{8}$ representation, whereas the latter from $\mathrm{SU}(8)$-adj.. We see that the roots in the former contain $\pm \alpha_{7}$, and those in the latter do not.

All the above argument on the roots of irreducible components can be confirmed explicitly, by adopting a basis of the space of root lattice. Simple roots are given by (182) with $d=7$. Positive roots are

$$
\begin{aligned}
& L_{i}-L_{j} \quad(i<j), \\
& L_{0}-\left(L_{i}+L_{j}+L_{k}\right) \quad(i<j<k) \quad \text { and } \\
& 2 L_{0}+L_{k}-\left(L_{1}+\cdots+L_{7}\right) .
\end{aligned}
$$

The highest root is $\theta \equiv 2 L_{0}-\left(L_{2}+\cdots+L_{7}\right)=2 \alpha_{1}+3 \alpha_{2}+4 \alpha_{3}+3 \alpha_{4}+2 \alpha_{5}+\alpha_{6}+2 \alpha_{7}$. Each irreducible components of $\mathfrak{e}_{7}$ under the subalgebra $\mathfrak{s u}(5)+\mathfrak{s u}(2)_{2}+\mathfrak{u}(1)+\mathfrak{u}(1)$ consists of the following roots:

- (1,adj.): $\pm \alpha_{1}= \pm\left(L_{1}-L_{2}\right)$.

- $(\operatorname{adj} . \oplus 5 \oplus \overline{\mathbf{5}}, \mathbf{1})$ :

$$
\begin{aligned}
& \diamond \pm\left(L_{i}-L_{j}\right) \quad(3 \leq i<j \leq 7) \\
& \diamond+\left(L_{0}-\left(L_{1}+L_{2}\right)-L_{k}\right) \quad(k=3, \cdots, 7),
\end{aligned}
$$

The lowest weight is $\alpha_{7} \quad(k=3), \quad[$ for $\bar{H}(\overline{\mathbf{5}})$ in ID A].

$$
\diamond-\left(L_{0}-\left(L_{1}+L_{2}\right)-L_{k}\right) \quad(k=3, \cdots, 7), \quad \text { [for nothing]. }
$$

- $\overline{\left(\wedge^{2} 5 \oplus 5,2\right)}$ :

$\diamond L_{0}-\left(L_{a}+L_{i}+L_{j}\right) \quad(a=1,2$ and $3 \leq i<j \leq 7)$.

The lowest weights are a doublet $\left(\alpha_{7}+\alpha_{1}+\alpha_{2}+\alpha_{3}, \alpha_{7}+\alpha_{2}+\alpha_{3}\right)$, [for Hermitian conjugate of $\mathbf{1 0}=(Q, \bar{U}, \bar{E})]$. 
$\diamond L_{a}-L_{k}$ for $k=3, \ldots, 7, \quad[$ for $\overline{\mathbf{5}}=(\bar{D}, L)$ in ID A].

The lowest weights are a doublet $\left(\alpha_{1}+\alpha_{2}, \alpha_{2}\right)$.

- $\overline{\left(\wedge^{3} \boldsymbol{5} \oplus \wedge^{4} \mathbf{5}, \mathbf{1}\right)}$ :

$\diamond L_{0}-\left(L_{i}+L_{j}+L_{k}\right) \quad(3 \leq i<j<k \leq 7), \quad$ [for nothing], including $\theta-\left(\alpha_{7}+\alpha_{1}+\alpha_{2}+\alpha_{3}\right)=\left(\alpha_{1}+2 \alpha_{2}+\alpha_{7}\right)+\left(3 \alpha_{3}+3 \alpha_{4}+2 \alpha_{5}+\alpha_{6}\right)$.

$\diamond 2 L_{0}-\left(L_{1}+\cdots+L_{7}\right)+L_{k} \quad(k=3, \cdots, 7), \quad[$ for $H(5)]$.

The highest weight is $\theta-\alpha_{1}-\alpha_{2}=\left(\alpha_{1}+2 \alpha_{2}+2 \alpha_{7}\right)+\left(4 \alpha_{3}+3 \alpha_{4}+2 \alpha_{5}+\alpha_{6}\right)$,

The lowest weight is $\left(\alpha_{1}+2 \alpha_{2}+2 \alpha_{7}\right)+\left(3 \alpha_{3}+2 \alpha_{4}+\alpha_{5}\right)=2 L_{0}-\left(L_{1}+\cdots+L_{6}\right)$.

- $\overline{\left(\wedge^{6} \mathbf{6}, \mathbf{2}\right)}$ : [for Hermitian conjugate of $\bar{N}$ in ID A]

$$
\begin{aligned}
& 2 L_{0}-\left(L_{1}+\cdots+L_{7}\right)+L_{1}=\left(2 \alpha_{1}+3 \alpha_{2}\right)+\left(4 \alpha_{3}+3 \alpha_{4}+2 \alpha_{5}+\alpha_{6}+2 \alpha_{7}\right)=\theta, \\
& 2 L_{0}-\left(L_{1}+\cdots+L_{7}\right)+L_{2}=\left(\alpha_{1}+3 \alpha_{2}\right)+\left(4 \alpha_{3}+3 \alpha_{4}+2 \alpha_{5}+\alpha_{6}+2 \alpha_{7}\right)=\theta-\alpha_{1} .
\end{aligned}
$$

\section{A.3 $\mathfrak{e}_{8}$ Lie algebra}

The simple roots of $\mathfrak{e}_{8}$ Lie algebra is given by (182) with $d=8$. Positive roots are

$$
\begin{aligned}
& L_{i}-L_{j} \quad(i<j), \\
& L_{0}-\left(L_{i}+L_{j}+L_{k}\right) \quad(i<j<k), \\
& 2 L_{0}+\left(L_{i}+L_{j}\right)-\left(L_{1}+\cdots+L_{8}\right) \quad(i<j) \text { and } \\
& 3 L_{0}-L_{k}-\left(L_{1}+\cdots+L_{8}\right),
\end{aligned}
$$

and the maximal root $\theta$ satisfies

$$
\theta=2 \alpha_{1}+4 \alpha_{2}+6 \alpha_{3}+5 \alpha_{4}+4 \alpha_{5}+3 \alpha_{6}+2 \alpha_{7}+3 \alpha_{8}=3 L_{0}-\left(L_{1}+\cdots+L_{8}\right)-L_{8} .
$$

The $\mathfrak{s u}(6)_{1}+\mathfrak{s u}(2)_{1}+\mathfrak{s u}(2)_{2}+\mathfrak{u}(1)$ subalgebra of $\mathfrak{e}_{8}$ is obtained by removing the odes of $\alpha_{2}$ and $\alpha_{7}$ from the extended Dynkin diagram of $E_{8} \cdot \mathfrak{s u}(2)_{1}$ is generated by the maximal root $\theta$, and its commutant $\mathfrak{e}_{7}$ by $\alpha_{1, \cdots, 6}$ and $\alpha_{8} \cdot \mathfrak{e}_{7}$ contains the $\mathfrak{s u}(6)_{1}+\mathfrak{s u}(2)_{2}+\mathfrak{u}(1)$ subalgebra. Brute force calculation shows that the irreducible components of $\mathfrak{e}_{8}$ under the subalgebra $\mathfrak{s u}(5)_{\text {GUT }}+\mathfrak{s u}(2)_{1}+\mathfrak{s u}(2)_{2}$ are: 
- $\mathfrak{s u}(5)_{\text {GUT }}$-singlets:

$$
\begin{aligned}
& \left(\mathbf{1} \oplus \mathbf{2}_{1} \oplus \mathbf{2}_{2}\right) \otimes\left(\mathbf{1} \oplus \mathbf{2}_{1} \oplus \mathbf{2}_{2}\right)^{*}= \\
& \left(\begin{array}{c|cc|cc} 
& \alpha^{\prime \prime} & & & \\
\hline \alpha^{\prime \prime} & & -\theta & & \\
L_{0}-\left(L_{1}+L_{2}+L_{8}\right) & \theta & & \alpha^{\prime} & \alpha^{\prime}+\alpha_{1} \\
\hline \alpha^{\prime \prime \prime}+\alpha_{1} & L_{1}-L_{8} & -\alpha^{\prime} & & \alpha_{1} \\
\alpha^{\prime \prime \prime} & L_{2}-L_{8} & -\alpha^{\prime}-\alpha_{1} & -\alpha_{1} &
\end{array}\right), \\
& \alpha^{\prime \prime} \equiv 2 L_{0}-\left(L_{3}+\cdots+L_{8}\right)=2 \alpha_{1}+4 \alpha_{2}+\alpha_{7}+2 \alpha_{8}+\left(5 \alpha_{3}+4 \alpha_{4}+3 \alpha_{3}+2 \alpha_{6}\right), \\
& \alpha^{\prime} \equiv 3 L_{0}-\left(2 L_{1}+L_{2}+\cdots+L_{8}\right)=\alpha_{1}+3 \alpha_{2}+\alpha_{7}+3 \alpha_{8}+\left(5 \alpha_{3}+4 \alpha_{4}+3 \alpha_{3}+2 \alpha_{6}\right) \text {, } \\
& \alpha^{\prime \prime \prime} \equiv-2 L_{0}+\left(L_{2}+\cdots+L_{7}\right)=-\left(2 \alpha_{1}+3 \alpha_{2}+2 \alpha_{8}\right)+\cdots \text {, }
\end{aligned}
$$

- $\mathfrak{s u}(5)_{\mathrm{GUT}}-\mathbf{1 0}$ representations:

$$
\left(\wedge^{2} \mathbf{1 0},\left(\mathbf{1} \oplus \mathbf{2}_{1} \oplus \mathbf{2}_{2}\right)\right)=\left\{L_{i}+L_{j}+\left(\frac{\frac{L_{0}-\left(L_{3}+\cdots+L_{7}\right)}{-L_{0}+L_{8}}}{\frac{2 L_{0}-\left(L_{1}+\cdots+L_{8}\right)}{-L_{0}+L_{1}}}\right)\right\}_{3 \leq i<j \leq 7},
$$

- $\mathfrak{s u}(5)_{\mathrm{GUT}}-\overline{5}$ representations:

$$
\begin{aligned}
& \left(\overline{\mathbf{5}}, \wedge^{2}\left(\mathbf{1} \oplus \mathbf{2}_{1} \oplus \mathbf{2}_{2}\right)\right)= \\
& \left\{\begin{array}{c|cc|c} 
& & \\
-L_{k}+\left(\begin{array}{ccc} 
\\
L_{8}
\end{array}\right. & & L_{0}-\left(L_{1}+L_{2}\right) & \\
L_{8}+\theta & & & \\
\hline L_{1} & L_{8}-\alpha^{\prime} & L_{0}-\left(L_{8}+L_{2}\right) & \alpha^{\prime \prime \prime}+L_{1} \\
L_{2} & L_{8}-\alpha^{\prime}-\alpha_{1} & L_{0}-\left(L_{8}+L_{1}\right) &
\end{array}\right\}_{k=3, \cdots, 7} .
\end{aligned}
$$

Two different $\mathrm{SU}(6)$ subgroups, $\mathrm{SU}(6)_{1}$ and $\mathrm{SU}(6)_{2}$, appear in the main text. They are both subgroups of $\mathrm{SO}(12)$ in $E_{8} \supset \mathrm{SO}(12) \times \mathrm{SU}(2)_{1} \times \mathrm{SU}(2)_{2}$. The irreducible components in

$$
\begin{aligned}
\operatorname{Res}_{\mathrm{SO}(12) \times \mathrm{SU}(2)_{1} \times \mathrm{SU}(2)_{2}}^{E_{8}}\left(\mathfrak{e}_{8}-\mathbf{a d j} .\right) \simeq & (\mathbf{a d j} ., \mathbf{1}, \mathbf{1}) \oplus(\mathbf{1}, \mathbf{a d j} ., \mathbf{1}) \oplus(\mathbf{1}, \mathbf{1}, \mathbf{a d j} .) \\
& \oplus(\mathbf{1 2}, \mathbf{2}, \mathbf{2}) \oplus\left(\mathbf{3 2}^{\prime}, \mathbf{1}, \mathbf{2}\right) \oplus(\mathbf{3 2}, \mathbf{2}, \mathbf{1})
\end{aligned}
$$

reduces under $\mathrm{SU}(6)_{1} \times \mathrm{U}(1)_{q_{6}} \subset \mathrm{SO}(12)$ to

$$
\begin{aligned}
\operatorname{Res}_{\mathrm{SU}(6)_{1} \times \mathrm{U}(1)_{q_{6}}}^{\mathrm{SO}(12)} & \mathbf{3 2 ^ { \prime }} \simeq\left[\mathbf{1}^{3} \oplus \wedge^{2} \mathbf{6}^{1}\right] \oplus \text { h.c. } \\
\operatorname{Res}_{\mathrm{SU}(6)_{1} \times \mathrm{U}(1)_{q_{6}}}^{\mathrm{SO}} \mathbf{3 2} & \simeq \mathbf{6}^{2} \oplus \wedge^{3} \mathbf{6}^{0} \oplus \overline{\mathbf{6}}^{-2},
\end{aligned}
$$


and under $\mathrm{SU}(6)_{2} \times \mathrm{U}(1)_{\tilde{q}_{6}} \subset \mathrm{SO}(12)$ to

$$
\begin{aligned}
& \operatorname{Res}_{\mathrm{SU}(6)_{2} \times \mathrm{U}(1)_{\tilde{q}_{6}}}^{\mathrm{SO}(12)} \mathbf{3 2 ^ { \prime }} \simeq \mathbf{6}^{2} \oplus \wedge^{3} \mathbf{6}^{0} \oplus \overline{\mathbf{6}}^{-2} \\
& \operatorname{Res}_{\mathrm{SU}(6)_{2} \times \mathrm{U}(1)_{\tilde{q}_{6}}}^{\mathrm{SO}(12)} \mathbf{3 2} \simeq\left[\mathbf{1}^{3} \oplus \wedge^{2} \mathbf{6}^{1}\right] \oplus \text { h.c. }
\end{aligned}
$$

Two $\mathrm{SU}(3)$ subgroups, $\mathrm{SU}(3)_{1}$ and $\mathrm{SU}(3)_{2}$, contain $\mathrm{SU}(2)_{2} \times \mathrm{U}(1)_{q_{6}}$ and $\mathrm{SU}(2)_{1} \times \mathrm{U}(1)_{\tilde{q}_{6}}$, respectively, and

$$
\begin{aligned}
& \mathrm{SU}(6)_{1} \times \mathrm{SU}(3)_{1} \times \mathrm{SU}(2)_{1} \subset E_{8}, \\
& \mathrm{SU}(6)_{2} \times \mathrm{SU}(3)_{2} \times \mathrm{SU}(2)_{2} \subset E_{8} .
\end{aligned}
$$

\section{B ALE spaces}

This section of the appendix is a quick summary of useful results.

The ALE spaces of $A_{r}, D_{r}$ and $E_{r}$ types are constructed by hyper-Kähler quotient [52, 80] as vacuum moduli of quiver gauge theories associated with the extended Dynkin diagrams of the simply laced Lie algebras. Their moduli parameters are the Fayet-Iliopoulos parameters of the sigma model in physics terminology or the values of the moment maps in mathematical terminology. The Fayet-Iliopoulos parameters $\vec{\zeta}^{i}=\left(\zeta_{1}^{i}, \zeta_{2}^{i}, \zeta_{3}^{i}\right)$ for $i=0, \cdots, r$ have to satisfy a "traceless condition"

$$
n_{i} \vec{\zeta}^{i}=0
$$

where integers $n_{i}$ (sometimes denoted as $a_{i}$ ) are the labels of the extended Dynkin diagrams; for the simple roots $\alpha_{i}(i=1, \cdots, r)$,

$$
n_{i} \alpha_{i}=0
$$

where $\alpha_{0} \equiv-\theta$ is the negative of the highest root and $n_{0} \equiv 1$.

The metric is known for the $A_{n-1}$-type and $D_{n}$ type. The ALE spaces are described as $S^{1}$ fibration (coordinate $\tau$ ) over a three dimensional space (coordinates $\vec{x}=\left(x_{1}, x_{2}, x_{3}\right)$ ) and the metric of $A_{n-1}$ type and $D_{n}$ type is specified by $\vec{r}_{i}=\left(r_{1}, r_{2}, r_{3}\right)_{i}$ for $i=1, \cdots, n$. The $S^{1}$ fibre shrinks at $\vec{x}=\vec{r}_{i}$ in $A_{n-1}$ type, and at $\vec{x}=\vec{r}_{i}$ and $\vec{x}=-\vec{r}_{i}$ in $D_{n}$ type. Between two

such points, there is a topological 2-cycle, given by $S^{1}$ fibration over the interval between the two points. The Fayet-Iliopoulos parameters $\vec{\zeta}^{i}$ describing the ALE spaces are given in terms of $\vec{r}_{i}$ by

$$
\vec{\zeta}^{i}=\vec{r}_{i}-\vec{r}_{i+1} \quad(i=0, \cdots, n-1)
$$


for $A_{n-1}$ case, where $i=0=n$ is understood, and

$$
\begin{array}{cc}
\vec{\zeta}^{0}=-\vec{r}_{1}-\vec{r}_{2}, \quad \vec{\zeta}^{i}=\vec{r}_{i}-\vec{r}_{i+1} \quad \vec{\zeta}^{n}=\vec{r}_{n-1}+\vec{r}_{n}, \\
\vec{\zeta}^{1}=\vec{r}_{1}-\vec{r}_{2}, \quad(\text { for } i=2, \cdots, n-2), & \vec{\zeta}^{n-1}=\vec{r}_{n-1}-\vec{r}_{n},
\end{array}
$$

for $D_{n}$ case. It is easy to see that the constraint (208) is satisfied for the $A_{n-1}$ and $D_{n}$ cases; note that $n_{i}=1$ for ${ }^{\forall} i$ in $A_{n-1}$, and $n_{i}=2$ in $D_{n}$ except $n_{0}=n_{1}=n_{n-1}=n_{n}=1$. The data $\vec{r}_{i}(i=1, \cdots, n)$ correspond to the location of $n$ D6-branes in their transverse directions in the Type IIA interpretation, if the ALE space of $A_{n-1}$ or $D_{n}$ type is used as the background geometry of the M-theory [81].

When the 2-cycle associated with the root $-\theta$ shrinks, the first D6-brane comes on top the $n$-th D6-brane in the $A_{n-1}$ case, and an $\mathrm{SU}(2)$ symmetry is enhanced. In the case of $D_{n}$ type ALE space, the first D6-brane moves across an O6-plane and comes on top of the orientifold image of the second D6-brane, and an SU(2) symmetry is enhanced [81]. Thus, 2-cycles involving the 2-cycle for $-\theta$ can be treated at the equal footing as other 2-cycles. In section 4, we see that massless matters arising from vanishing $C_{-\theta}$ cycle are identified with the $H(5)$ multiplet in the model with $E_{6}$ underlying symmetry, and with right-handed

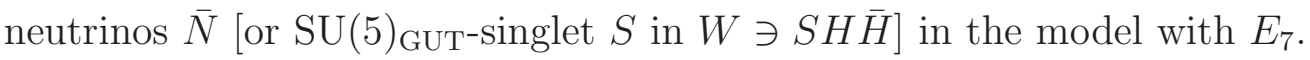

\section{Acknowledgements}

We thank Ralph Blumenhagen, Alon Faraggi, Yang-Hui He, Karl Landsteiner, Thomas Mohaupt, Michael Schulz, Eric Sharpe and Timo Weigand for fruitful discussion and correspondence. This work was supported in part by a PPARC Advanced Fellowship (R.T.), and in part by the Director, Office of Science, Office of High Energy and Nuclear Physics, of the US Department of Energy under Contract DE-AC02-05CH11231, and in part by the Miller Intitute for Basic Research in Science (T.W.).

\section{References}

[1] A. E. Faraggi, D. V. Nanopoulos and K. j. Yuan, "A Standard Like Model In The 4-D Free Fermionic String Formulation," Nucl. Phys. B 335, 347 (1990).

[2] A. E. Faraggi, "Hierarchical top - bottom mass relation in a superstring derived standard - like model," Phys. Lett. B 274, 47 (1992), "Construction of realistic standard - like 
models in the free fermionic superstring formulation," Nucl. Phys. B 387, 239 (1992) arXiv:hep-th/9208024, "A New standard - like model in the four-dimensional free fermionic string formulation,"Phys. Lett. B 278, 131 (1992);

G. B. Cleaver, A. E. Faraggi and D. V. Nanopoulos, "String derived MSSM and M-theory unification," Phys. Lett. B 455, 135 (1999) arXiv:hep-ph/9811427.

[3] M. Cvetic, G. Shiu and A. M. Uranga, "Chiral four-dimensional N = 1 supersymmetric type IIA orientifolds from intersecting D6-branes," Nucl. Phys. B 615, 3 (2001) arXiv:hep-th/0107166, "Chiral type II orientifold constructions as M theory on G(2) holonomy spaces," arXiv:hep-th/0111179.

M. Cvetic, I. Papadimitriou and G. Shiu, "Supersymmetric three family SU(5) grand unified models from type IIA orientifolds with intersecting D6-branes," Nucl. Phys. B 659, 193 (2003) [Erratum-ibid. B 696, 298 (2004)] arXiv:hep-th/0212177.

[4] V. Braun, B. A. Ovrut, T. Pantev and R. Reinbacher, "Elliptic Calabi-Yau threefolds with Z(3) x Z(3) Wilson lines," JHEP 0412 (2004) 062 arXiv:hep-th/0410055;

V. Braun, Y. H. He, B. A. Ovrut and T. Pantev, "The exact MSSM spectrum from string theory," arXiv:hep-th/0512177;

V. Braun, Y. H. He and B. A. Ovrut, "Yukawa couplings in heterotic standard models," arXiv:hep-th/0601204.

[5] R. Blumenhagen, M. Cvetic, P. Langacker and G. Shiu, "Toward realistic intersecting D-brane models," arXiv:hep-th/0502005.

[6] W. Buchmuller, K. Hamaguchi, O. Lebedev and M. Ratz, "The supersymmetric standard model from the heterotic string," arXiv:hep-ph/0511035.

[7] V. Bouchard and R. Donagi, "An SU(5) heterotic standard model," Phys. Lett. B 633, 783 (2006) arXiv:hep-th/0512149.

[8] V. Bouchard, M. Cvetic and R. Donagi, "Tri-linear Couplings in an Heterotic Minimal Supersymmetric Standard Model," arXiv:hep-th/0602096.

[9] N. S. Manton, "A New Six-Dimensional Approach To The Weinberg-Salam Model," Nucl. Phys. B 158, 141 (1979);

D. B. Fairlie, "Higgs' Fields And The Determination Of The Weinberg Angle," Phys. Lett. B 82, 97 (1979),

"Two Consistent Calculations Of The Weinberg Angle," J. Phys. G 5, L55 (1979);

P. Forgacs and N. S. Manton, "Space-Time Symmetries In Gauge Theories," Commun. 
Math. Phys. 72, 15 (1980);

S. Randjbar-Daemi, A. Salam and J. A. Strathdee, "Spontaneous Compactification In Six-Dimensional Einstein-Maxwell Theory," Nucl. Phys. B 214, 491 (1983).

[10] W. Buchmuller, S. T. Love, R. D. Peccei and T. Yanagida, "Quasi Goldstone Fermions," Phys. Lett. B 115 (1982) 233;

C. L. Ong, "Gauged Supersymmetric Generalized Nonlinear Sigma Models For Quarks And Leptons," Phys. Rev. D 27, 3044 (1983);

T. Kugo and T. Yanagida, "Unification Of Families Based On A Coset Space E7 / SU(5) X SU(3) X U(1)," Phys. Lett. B 134, 313 (1984).

[11] P. Candelas, G. T. Horowitz, A. Strominger and E. Witten, "Vacuum Configurations For Superstrings," Nucl. Phys. B 258, 46 (1985).

[12] T. Yanagida and J. Sato, "Large lepton mixing in seesaw models: Coset-space family unification," Nucl. Phys. Proc. Suppl. 77, 293 (1999) arXiv:hep-ph/9809307;

P. Ramond, "Neutrinos: A glimpse beyond the standard model," Nucl. Phys. Proc. Suppl. 77, 3 (1999) arXiv:hep-ph/9809401.

See also C. H. Albright and S. M. Barr, "Fermion masses in $\mathrm{SO}(10)$ with a single adjoint Higgs field," Phys. Rev. D 58, 013002 (1998) arXiv:hep-ph/9712488, "A minimality condition and atmospheric neutrino oscillations," Phys. Rev. Lett. 81, 1167 (1998) arXiv:hep-ph/9802314,

[13] L. J. Hall, H. Murayama and N. Weiner, "Neutrino mass anarchy," Phys. Rev. Lett. 84, 2572 (2000) arXiv:hep-ph/9911341;

N. Haba and H. Murayama, "Anarchy and hierarchy," Phys. Rev. D 63, 053010 (2001) arXiv:hep-ph/0009174.

[14] T. Yanagida, in Proc. Workshop on the Unified Theory and the Baryon Number in the Universe, Tsukuba, 1979, eds. O. Sawada and S. Sugamoto, Report KEK-79-18 (1979); M. Gell-mann, P. Ramond and R. Slansky, in "Supergravity" North-Holland, Amsterdam, 1979, eds. D.Z. Freedman and P. van Nieuwenhuizen.

[15] E. Witten, "String theory dynamics in various dimensions," Nucl. Phys. B 443, 85 (1995) arXiv:hep-th/9503124.

[16] C. Vafa, "Evidence for F-Theory," Nucl. Phys. B 469 (1996) 403 arXiv:hep-th/9602022.

[17] D. R. Morrison and C. Vafa, "Compactifications of F-Theory on Calabi-Yau Threefolds - I," Nucl. Phys. B 473, 74 (1996) arXiv:hep-th/9602114 
[18] D. R. Morrison and C. Vafa, "Compactifications of F-Theory on Calabi-Yau Threefolds - II," Nucl. Phys. B 476 (1996) 437 arXiv:hep-th/9603161.

[19] R. Friedman, J. Morgan and E. Witten, "Vector bundles and F theory," Commun. Math. Phys. 187 (1997) 679 arXiv:hep-th/9701162.

[20] M. Bershadsky, A. Johansen, T. Pantev and V. Sadov, "On four-dimensional compactifications of F-theory," Nucl. Phys. B 505 (1997) 165 arXiv:hep-th/9701165.

[21] M. Bershadsky,V. Sadov and C. Vafa "D-branes and Topological Field Thoeries" Nucl. Phys. B463, 420(1996) arXiv:hep-th/9511222.

[22] M. R. Gaberdiel and B. Zwiebach, "Exceptional groups from open strings," Nucl. Phys. B 518 (1998) 151 arXiv:hep-th/9709013.

[23] [Particle Data Group] S. Eidelman et al., Phys. Lett. B592, 1 (2004).

[24] N. Arkani-Hamed, T. Gregoire and J. Wacker, "Higher dimensional supersymmetry in 4D superspace," JHEP 0203 (2002) 055 arXiv:hep-th/0101233.

[25] S. Kachru and C. Vafa, "Exact results for $\mathrm{N}=2$ compactifications of heterotic strings," Nucl. Phys. B 450, 69 (1995) arXiv:hep-th/9505105.

[26] M. Bershadsky, K. A. Intriligator, S. Kachru, D. R. Morrison, V. Sadov and C. Vafa, "Geometric singularities and enhanced gauge symmetries," Nucl. Phys. B 481 (1996) 215 arXiv:hep-th/9605200.

[27] J. R. Ellis, J. F. Gunion, H. E. Haber, L. Roszkowski and F. Zwirner, "Higgs Bosons In A Nonminimal Supersymmetric Model," Phys. Rev. D 39 (1989) 844.

[28] P. Ciafaloni and A. Pomarol, "Dynamical determination of the supersymmetric Higgs mass," Phys. Lett. B 404, 83 (1997) arXiv:hep-ph/9702410;

L. J. Hall and T. Watari, "Electroweak supersymmetry with an approximate U(1)(PQ)," Phys. Rev. D 70, 115001 (2004) arXiv:hep-ph/0405109;

D. J. Miller and R. Nevzorov, "The Peccei-Quinn axion in the next-to-minimal supersymmetric standard model," arXiv:hep-ph/0309143,

B. Feldstein, L. J. Hall and T. Watari, "Simultaneous solutions of the strong CP and mu problems," Phys. Lett. B 607, 155 (2005) arXiv:hep-ph/0411013;

D. J. Miller, S. Moretti and R. Nevzorov, "Higgs bosons in the NMSSM with exact and slightly broken PQ-symmetry," arXiv:hep-ph/0501139.

[29] B. A. Dobrescu, G. Landsberg and K. T. Matchev, "Higgs boson decays to CP-odd scalars at the Tevatron and beyond," Phys. Rev. D 63, 075003 (2001) 
arXiv:hep-ph/0005308;

B. A. Dobrescu and K. T. Matchev, "Light axion within the next-to-minimal supersymmetric standard model," JHEP 0009, 031 (2000) arXiv:hep-ph/0008192;

R. Dermisek and J. F. Gunion, "Consistency of LEP event excesses with an $\mathrm{h} \rightarrow$ a a decay scenario and low-fine-tuning NMSSM models," arXiv:hep-ph/0510322,

S. Chang, P. J. Fox and N. Weiner, "Naturalness and Higgs decays in the MSSM with a singlet," arXiv:hep-ph/0511250

[30] E. Witten, "Symmetry Breaking Patterns In Superstring Models," Nucl. Phys. B 258, 75 (1985).

[31] A. Masiero, D. V. Nanopoulos, K. Tamvakis and T. Yanagida, "Naturally Massless Higgs Doublets In Supersymmetric SU(5)," Phys. Lett. B 115, 380 (1982).

[32] T. Watari and T. Yanagida, "Product-group unification in type IIB string thoery," Phys. Rev. D 70, 036009 (2004) arXiv:hep-ph/0402160.

[33] K. I. Izawa and T. Yanagida, "R-invariant natural unification," Prog. Theor. Phys. 97, 913 (1997) arXiv:hep-ph/9703350.

[34] R. Donagi, Y. H. He, B. A. Ovrut and R. Reinbacher, "The particle spectrum of heterotic compactifications," JHEP 0412, 054 (2004) arXiv:hep-th/0405014.

[35] R. Blumenhagen, G. Honecker and T. Weigand, "Loop-corrected compactifications of the heterotic string with line bundles," JHEP 0506 (2005) 020 arXiv:hep-th/0504232.

[36] F. Hirzebruch, "Topological Methods in Algebraic Geometry," Springer (1978).

[37] R. Blumenhagen, S. Moster and T. Weigand, "Heterotic GUT and Standard Model vacua from simply connected Calabi-Yau manifolds," arXiv:hep-th/0603015.

[38] E. Witten, “New Issues In Manifolds Of SU(3) Holonomy,” Nucl. Phys. B 268, 79 (1986).

[39] E. Witten, "Some Properties Of O(32) Superstrings," Phys. Lett. B 149, 351 (1984).

[40] M. Fukugita and T. Yanagida, "Baryogenesis Without Grand Unification," Phys. Lett. B 174, 45 (1986).

[41] K. Dick, M. Lindner, M. Ratz and D. Wright, "Leptogenesis with Dirac neutrinos," Phys. Rev. Lett. 84, 4039 (2000) arXiv:hep-ph/9907562. H. Murayama and A. Pierce, "Realistic Dirac leptogenesis," Phys. Rev. Lett. 89, 271601 (2002) arXiv:hep-ph/0206177.

[42] M. Dine, N. Seiberg, X. G. Wen and E. Witten, "Nonperturbative Effects On The String World Sheet," Nucl. Phys. B 278, 769 (1986) 
[43] J. P. Derendinger, L. E. Ibanez and H. P. Nilles, "On The Low-Energy Limit Of Superstring Theories," Nucl. Phys. B 267, 365 (1986).

[44] G. Aldazabal, L. E. Ibanez, F. Quevedo and A. M. Uranga, "D-branes at singularities: A bottom-up approach to the string embedding of the standard model," JHEP 0008, 002 (2000) arXiv:hep-th/0005067.

[45] H. Verlinde and M. Wijnholt, "Building the standard model on a D3-brane," arXiv:hep-th/0508089.

[46] E. Witten, "Deconstruction, G(2) holonomy, and doublet-triplet splitting," arXiv:hep-ph/0201018,

T. Friedmann and E. Witten, "Unification scale, proton decay, and manifolds of G(2) holonomy," Adv. Theor. Math. Phys. 7, 577 (2003) arXiv:hep-th/0211269;

I. R. Klebanov and E. Witten, "Proton decay in intersecting D-brane models," Nucl. Phys. B 664, 3 (2003) arXiv:hep-th/0304079;

M. Axenides, E. Floratos and C. Kokorelis, "SU(5) unified theories from intersecting branes," JHEP 0310, 006 (2003) arXiv:hep-th/0307255. B. S. Acharya and R. Valandro, "Suppressing proton decay in theories with localised fermions," arXiv:hep-ph/0512144.

[47] S. Katz and C. Vafa, "Matter from geometry," Nucl. Phys. B 497, 146 (1997) arXiv:hep-th/9606086.

[48] M. Atiyah and E. Witten "M-theory dynamics on a manifold of G(2) holonomy," Adv Theor. Math. Phys. 6, 1 (2003) arXiv:hep-th/0107177

[49] B. Acharya and E. Witten, "Chiral Fermions from manifolds of G(2) holonomy," arXiv:hep-th/0109152

[50] B. S. Acharya and S. Gukov, "M theory and Singularities of Exceptional Holonomy Manifolds," Phys. Rept. 392, 121 (2004) arXiv:hep-th/0409191.

[51] B. S. Acharya, "On realising $\mathrm{N}=1$ super Yang-Mills in $\mathrm{M}$ theory," arXiv:hep-th/0011089.

[52] P. B. Kronheimer, "The Construction Of Ale Spaces As Hyperkahler Quotients," J. Diff. Geom. 29 (1989) 665.

[53] M. Berkooz, M. R. Douglas and R. G. Leigh, "Branes intersecting at angles," Nucl. Phys. B 480, 265 (1996) arXiv:hep-th/9606139. 
[54] M. Cvetic, P. Langacker and G. Shiu, "A three-family standard-like orientifold model: Yukawa couplings and hierarchy," Nucl. Phys. B 642, 139 (2002) arXiv:hep-th/0206115.

[55] P. Berglund and A. Brandhuber, "Matter from G(2) manifolds", Nucl. Phys. B 641, 351 (2002) arXiv:hep-th/0205184.

[56] P. Berglund and P. Mayr, "Heterotic string/F-theory duality from mirror symmetry," Adv. Theor. Math. Phys. 2, 1307 (1999) arXiv:hep-th/9811217.

[57] G. Rajesh, "Toric geometry and F-theory/heterotic duality in four dimensions," JHEP 9812, 018 (1998) arXiv:hep-th/9811240.

[58] R. Hartshorne, "Algebraic Geometry," Springer (1977).

[59] B. Andreas and D. Hernandez Ruiperez, "U(n) vector bundles on Calabi-Yau threefolds for string theory compactifications," arXiv:hep-th/0410170.

[60] B. A. Ovrut, T. Pantev and J. Park, "Small instanton transitions in heterotic M-theory," JHEP 0005, 045 (2000) arXiv:hep-th/0001133.

[61] G. Curio, "Chiral matter and transitions in heterotic string models," Phys. Lett. B 435, 39 (1998) arXiv:hep-th/9803224;

D. E. Diaconescu and G. Ionesei, "Spectral covers, charged matter and bundle cohomology," JHEP 9812, 001 (1998) arXiv:hep-th/9811129.

[62] S. Sethi, C. Vafa and E. Witten, "Constraints on low-dimensional string compactifications," Nucl. Phys. B 480, 213 (1996) arXiv:hep-th/9606122.

[63] H. Jockers and J. Louis, "The effective action of D7-branes in N = 1 Calabi-Yau orientifolds," Nucl. Phys. B 705, 167 (2005) arXiv:hep-th/0409098.

[64] R. Blumenhagen, G. Honecker and T. Weigand, "Non-abelian brane worlds: The heterotic string story," JHEP 0510 (2005) 086 arXiv:hep-th/0510049.

[65] R. Friedman, J. W. Morgan and E. Witten, "Vector bundles over elliptic fibrations," arXiv:alg-geom/9709029.

[66] G. Curio and R. Y. Donagi, "Moduli in N = 1 heterotic/F-theory duality," Nucl. Phys. B 518 (1998) 603 arXiv:hep-th/9801057.

[67] V. Sadov, "Generalized Green-Schwarz mechanism in F theory," Phys. Lett. B 388 (1996) 45 arXiv:hep-th/9606008.

[68] A. Klemm, B. Lian, S. S. Roan and S. T. Yau, "Calabi-Yau fourfolds for M- and F-theory compactifications," Nucl. Phys. B 518, 515 (1998) arXiv:hep-th/9701023. 
[69] T. Gherghetta and A. Pomarol, "Bulk fields and supersymmetry in a slice of AdS," Nucl. Phys. B 586, 141 (2000) arXiv:hep-ph/0003129;

W. D. Goldberger, Y. Nomura and D. R. Smith, "Warped supersymmetric grand unification," Phys. Rev. D 67, 075021 (2003) arXiv:hep-ph/0209158.

[70] I. R. Klebanov and M. J. Strassler, "Supergravity and a confining gauge theory: Duality cascades and chiSB-resolution of naked singularities," JHEP 0008, 052 (2000) arXiv:hep-th/0007191.

[71] S. B. Giddings, S. Kachru and J. Polchinski, "Hierarchies from fluxes in string compactifications," Phys. Rev. D 66, 106006 (2002) arXiv:hep-th/0105097.

[72] K. Dasgupta, K. h. Oh, J. Park and R. Tatar, "Geometric transition versus cascading solution," JHEP 0201, 031 (2002) arXiv:hep-th/0110050; M. Becker, K. Dasgupta, S. Katz, A. Knauf and R. Tatar, "Geometric transitions, flops and non-Kaehler manifolds. II," Nucl. Phys. B 738, 124 (2006) arXiv:hep-th/0511099

[73] L. A. Pando Zayas and A. A. Tseytlin, "3-branes on resolved conifold," JHEP 0011, 028 (2000) arXiv:hep-th/0010088.

[74] F. Denef and M. R. Douglas, "Computational complexity of the landscape I," arXiv:hep-th/0602072.

[75] C. Vafa, "The string landscape and the swampland," arXiv:hep-th/0509212;

N. Arkani-Hamed, L. Motl, A. Nicolis and C. Vafa, "The string landscape, black holes and gravity as the weakest force," arXiv:hep-th/0601001.

[76] S. Kachru, J. McGreevy and P. Svrcek, "Bounds on masses of bulk fields in string compactifications," arXiv:hep-th/0601111.

[77] L. Kofman, A. Linde, X. Liu, A. Maloney, L. McAllister and E. Silverstein, "Beauty is attractive: Moduli trapping at enhanced symmetry points," JHEP 0405, 030 (2004) arXiv:hep-th/0403001.

[78] B. Feldstein, L. J. Hall and T. Watari, "Density perturbations and the cosmological constant from inflationary landscapes," Phys. Rev. D 72, 123506 (2005) arXiv:hep-th/0506235;

J. Garriga and A. Vilenkin, "Anthropic prediction for Lambda and the Q catastrophe," arXiv:hep-th/0508005;

A. Linde and V. Mukhanov, "The curvaton web," arXiv:astro-ph/0511736, 
L. J. Hall, T. Watari and T. T. Yanagida, "Taming the runaway problem of inflationary landscapes," arXiv:hep-th/0601028.

[79] M. R. Douglas, S. Katz and C. Vafa, "Small instantons, del Pezzo surfaces and type I' theory," Nucl. Phys. B 497, 155 (1997) arXiv:hep-th/9609071.

[80] M. R. Douglas and G. W. Moore, "D-branes, Quivers, and ALE Instantons," arXiv:hep-th/9603167.

[81] A. Sen, "A note on enhanced gauge symmetries in M- and string theory," JHEP 9709 (1997) 001 arXiv:hep-th/9707123. 